\title{
BEHAVIOUR OF THE CATTLE EGRET ARDEOLA IBIS
}

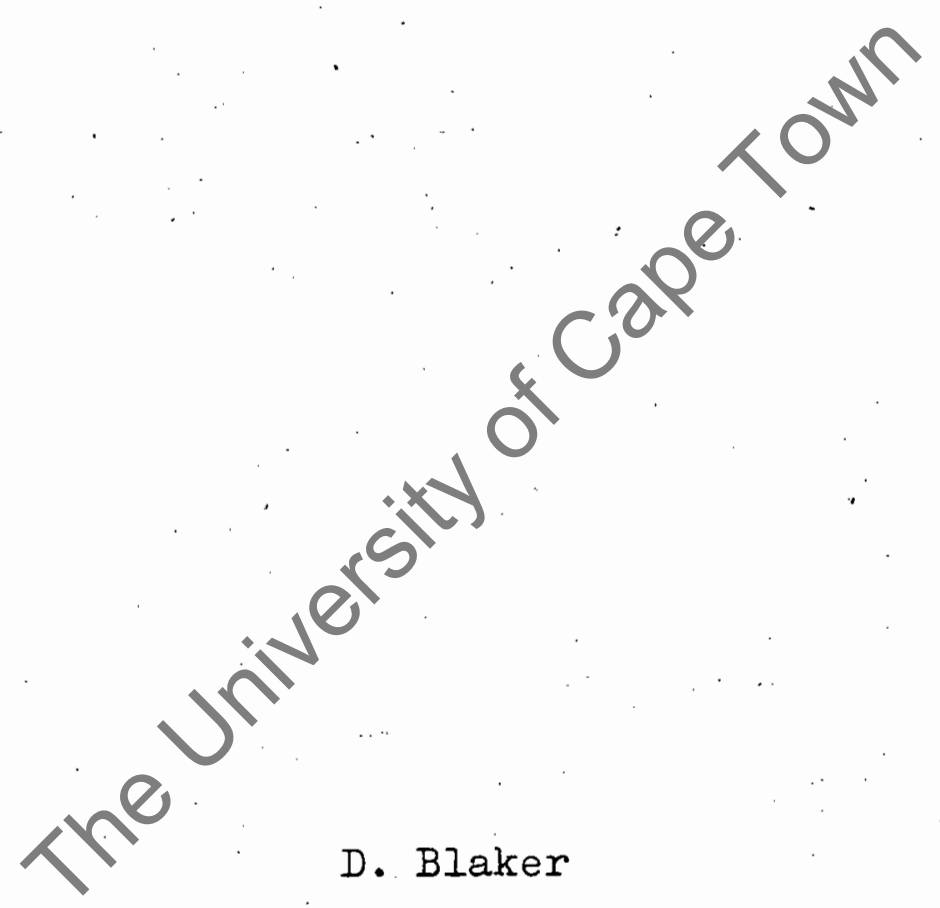

Submitted for the degree of M.Sc. in the Department of Zoology, University of Cape Town. April 1967. 
The copyright of this thesis vests in the author. No quotation from it or information derived from it is to be published without full acknowledgement of the source. The thesis is to be used for private study or noncommercial research purposes only.

Published by the University of Cape Town (UCT) in terms of the non-exclusive license granted to UCT by the author. 


\section{CONTENTS}

1. Introduction

1.1 Study Methods

1.2 Explanation of layout

2. Maintenance Activities

2.1 Flight

2.2 Feeding

2.3 Comfort Activities

2.4 Predation and Predator-responses

3. Displays

3.1 Introduction

3.11 Definitions

3.12 Display analysis

3.13 Display frequency

3.2 Agonistic Displays

3.21 Forward Display

3.22 Supplanting Run

3.23 Witharawn Crouch

3.24 Alert

3.3 Stretch Display

3.4 Flap Flight Display

3.5 Back-Biting

3.6 Twig Shake

3.7 Head Flick

3.8 Wing-Touch

3.9 Wing Spread

3.01 Greeting Ceremony

$3.02 \mathrm{Calls}$

3.021 Rick-rack

3.022 Chatter

3.023 Kok

3.024 Kaa日h

4. Non-breeding Behaviour

4.1 Daily routines and roosting behaviour

4.2 Flock sizes and host relationships

5. Breeding Behaviour

5.I Colour-and plumage-changes

5.2 Colony development

5.3 Interactions within the pair 
5.31 Mate-selection stage

5.32 Pair-formation stage

5.4 Copulation

5.5 Nest-building

5.51 Introduction

5.52 Techniques

5.53 Social aspects

5.6 Incubation

5.7 Nestling period

5.8 Attentiveness

5.81 Pair-formation stage

5.82 Incubation

5.83 Nestling period; Feeding rates

5.9 Interactions outside the pair

5.91 Aggression

5.92 Copulation between non-paired birds

6. Behaviour of young A. ibis

6.1 Maintenance activities

6.11 Hatching

6.12 Comfort movements

6.13 Behavioural thermoregulation

6.14 Climbing and the tendency to wander

6.15 Predator responses

6.16 Flying

6.2 Feeding and competition for food

6.21 Direct observation

6.22 Growth rates and chick mortality

6.23 Significance of beak colour

6.3 Displays and social interactions of the young

6.31 Interactions with adults

6.32 Interactions between chicks

6.33 Parent-young recognition

7. The behaviour of Egretta garzetta and E. intermedia

7.1 The behaviour of Egretta garzetta

7.11 Maintenance activities

7.12 Forward Displays

7.13 Calls

7.14 Snap Display and Twig Shake

7.15 Flight Displays

7.16 Stretch

7.17 Greeting ceremonies 
7.18 Bill-clappering

7.19 Head-flick, Wing-touch, Wing Spread

7.20 Breeding Behaviour

7.2 The behaviour of Egretta intermedia

7.21 Maintenance activities

7.22 Forward Dioplay

7.23 Calls

7.24 Twig Shake

7.25 Snap Display

7.26 Flight Displays

7.27 Stretch

7.28 Greeting ceremonies

7.29 Back-bite and Bill-clapper

7.30 Breeding behaviour

8. Discussion

9. References 


\section{INTRODUCTION}

Ardeola ibis (I.) is a small white heron, generally considered to be one of the most terrestrial members of its semi-aquatic family. It is a highly successful species, both in terms of numbers and of geographic range. Apart from man and his commensals, it is probably the commonest of the six terrestrial vertebrates with cosmopolitan breeding ranges, the other five all being bird species too (Voous 1960). A. ibis is gregarious, roosting and nesting in very large numbers, so one aim of this study is to investigate advantages of and adaptations to a social way of life, and to see whether the species' success is in any way a result of its gregariousness.

Several taxonomic reviews, notably those of Lorenz (1941) and Johnsgard (1961) on Anatidae, have been based on behavioural characters, because many behaviour patterns are as conservative and species-constant as structural features are. For this reason, and because adequate behavioural studies have to date been carried out on only about 7 of the 64 Ardeid species, the work was extended to include two other species, Egretta garzetta and E. intermedia. The present study is also designed to provide material for a projected review of the Ardeidae (Curry-Iindahl pers. comm.).

\section{I.I Study Methods}

This study consists primarily of broad-spectrum description, based entirely on field observation. In addition a limited number of experiments were carried out, chiefly on nestlings, to investigate some adaptive features. The majority of information was collected within a $50 \mathrm{~km}$. radius of Cape Town, with all the work on the species' breeding behaviour carried out at a large nesting colony (described in Section 5.1) near Paarl $\left(33^{\circ} 42^{\prime} \mathrm{s}\right.$, $\left.18^{\circ} 59^{\prime} \mathrm{W}\right)$, during August-November 1965 and 1966. Most observations were made at close range from hides, which were usually built before the surrounding nests were, thus reducing the chance of the birds having a disturbing influence imposed on them. In order to facilitate individual recognition 26 adults were marked with a dye (printer's ink solution) sprayed at them from a hide. This gave each bird a distinctive pattern of markings and had the advantage over ringing of involving minimum 
disturbance, and enabling sitting birds to be easily recognised.

The sexes are morphologically very similar in A. ibis and this provided a major obstacle at first. Although no measurements are available, it is quite obvious that during the breeding season the plumes of males are on the average longer and more abundant than those of females, but there is considerable overlap so that the feature is of limited use. As will be described in Section 5.3 and 5.5, the behaviour of males and females is quite distinct during much of the pair-formation and pre-incubation stages. The most useful single criterion was the sexes' different behaviour during nest building, for over $99 \%$ of material is brought by the male and $96 \%$ actually inserted into the nest structure by the the fernale. Many of the individuals observed during the incubation period had been previously marked and sexed during copulation.

Most notes were kept in the form of time-scores (incorrectly named "ethograms" by Meyerriecks 1960 and Kahl 1966); some detailed, others just noting the incidence of various behaviour patterns. The individual histories of over 100 birds were followed for periods ranging from a few minutes up to 56 days. During the incubation and nestling stages observation times were standardised (0630 to 1230 hours and 1230 to 1830 on alternate days; at midday at Parrl is at 12.38 hours), chiefly in order to investigate the species' attentive patterns. In order to allow ample time for any disturbance among the birds to die down, the hide was always entered 20 to 30 minutes before observation was due to begin. Methods of display analysis and techniques of the experiments carried out on nestlings are described in section 3 and 6 .

\subsection{Explanation of layout}

A species' activities may be roughly divided into two groups. (a) Firstly, there are behaviour patterns concerned with the individual's efficiency; namely; locomotion, feeding, comfort movements, thernoregulation and avoiding predators. These are known as maintenance activities (Section 2). (b) Secondly, there is social behaviour, which includes displays and their use in territorial behaviour, mating behaviour, etc. The arbitrariness of the distinction between maintenance activities and displays is discussed in section 3.1 . 
Social behaviour has been further divided into display description and analysis (Section 3), an account of the behaviour during the non-breeding season (Section 4), and a detailed description of the complex social interactions which occur during the breeding season (section 5). The behaviour of young A. ibig has been dealt with separately in section 6 . 


\subsection{Flight}

In normal flight the birds flap at an average of 198 times per minute (20 30-second observations on different individuals, range 189 to 216). Figures on flight-speed are not presented here because it is impossible to accurately measure wind-speed at the necessary altitude. When approaching a roost from upwind or a feeding area surrounded by tall trees, A. ibis occasionally descend with a rapid side-slipping swerving flight. On one windless evening only 4 out of 384 individuals approached a roost with swerving flight. Swerving flight clearly serves to check the rate of descent when a bird is flying steeply downwards, but it is not always functional for birds have, on three occasions, been seen to perform swerving flight when leaving a roost. It occurs in several Ardeidae (Meyerricks 1960).. Birds approaching roosts in the evening occasionally fly in $\mathrm{V}$ formations but the habit is weakly developed. Figures on flock size are given in Section 4.

Daanje (195I) was the first to ascribe importance to intention movements of flight in display origin, and at least one Ardeid display (Stretch) is derived from this source. For this reason, high speed (64 f.p.s.) cine films were taken of 19 takeoff sequences, one of which is shown in Figure 1 . The sequence of skeletal movements is as follows: legs begin to bend, neck is partly retracted and forepart of the body lowered until the main body axis is about 300 above the horizontal, simultaneously the wings are raised and extended and as they beat down the legs are extended again. No tail movements relative to the body could be detected in any of the 19 sequences.

\section{$2.2 \quad$ Feeding}

A. ibis' normal method of hunting is a steady walk interspersed with agile runs at prey which has been sighted. (Food consists mainly of Arthropoda, with Orthoptera predominating, Siegfried 1966a). Birds repeatedly crane their necks to examine tufts of grass more closely. When an animal is located it is usually (details below) captured by a short run followed by a stab. Smaller prey is swallowed immediately but larger ones such as frogs and lizards are pecked to death, usually 

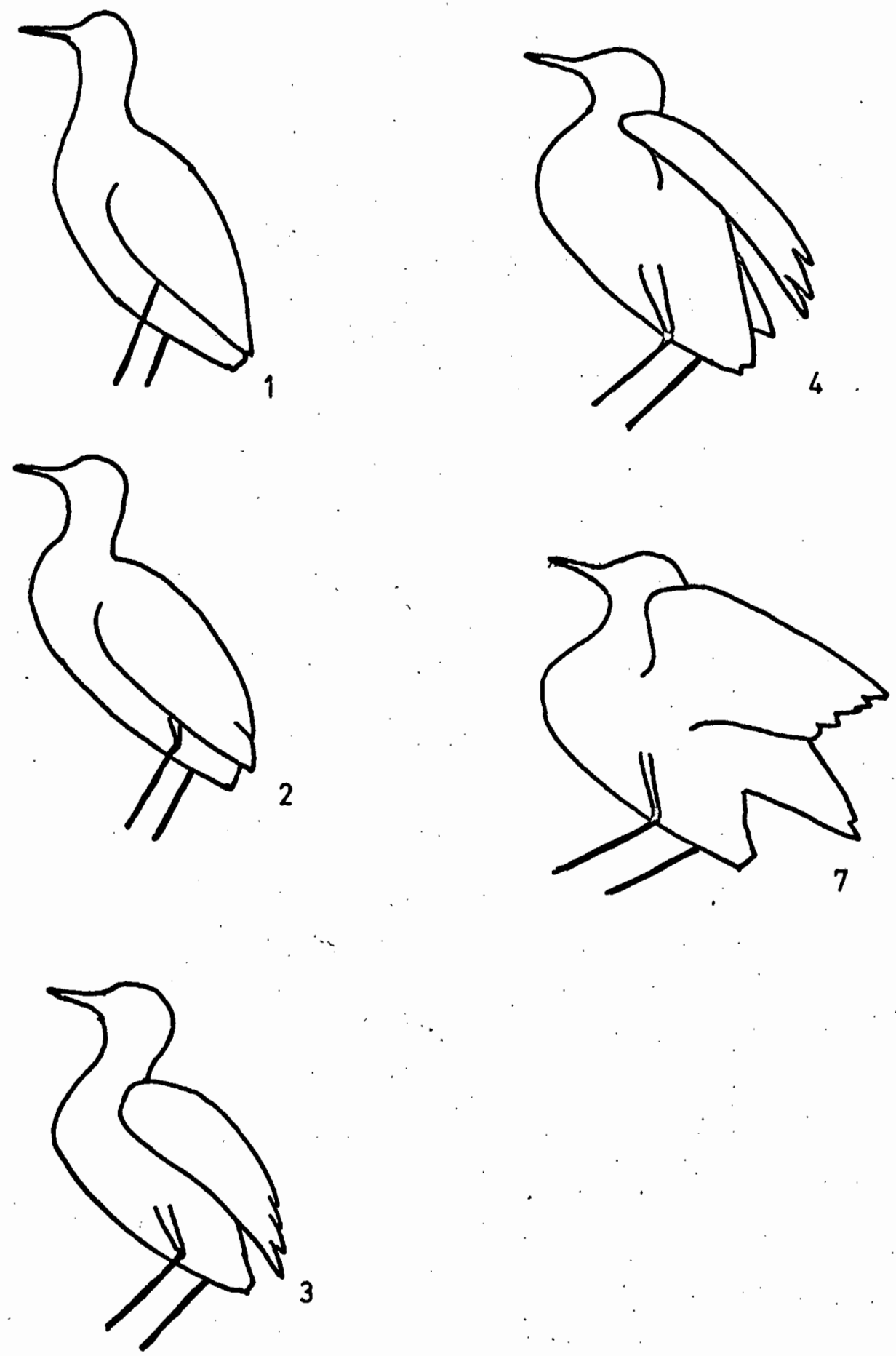

FIGURE 1. The movements of take-off, drawn from a $16 \mathrm{~mm}$. ciné sequence at 64 frames per second. Numbers denote frames in the sequence. 
dipped several times in water if available, and swallowed whole. Flying insects are sometimes chased and caught on the wing. A. ibis does not recognise prey animals by movement alone, for inanimate objects such as twigs are often pecked at, and pupae are sometimes eaten.

A less common form of feeding in A. ibis has been named Stand and Wait in Butorides virescens by Meyerriecks (1960). (Repeated reference will be made to this paper by Meyerriecks (1960), which will henceforth be referred to by the author's name only.) The bird stands motionless for up to a minute, neck outstretched at an angle of about $30^{\circ}$, while it peers intently at one spot (figure 2). Feeding flocks sometimes perform group movements similar to those which Ward (1966) names "roller feeding" in Quelea quelea. "The birds all advance in the same direction, with birds at the back continually flying over the heads of the others to feed in the front rank. The species commensalistic relationship with large herbivores is dealt with in section 4.1 .

A striking feature of feeding behaviour is neck-swaying. When an animal has been sighted, the bird extends its head and neck forward until its body is almost horizontal. It then begins to sway its head and neck from side to side, usually at a rate of about four per second at first, the rate of swaying increasing until the suduen forward and downward stab is made. This describes neck-swaying in its typical form, but the pattern varies greatly from slight neck-movements to a violent swaying in which the whole bodies shakes and the head moves through an arc of about $10 \mathrm{~cm}$. The pattern has been observed once in Egretta garzetta and has been described in Ardea melanocephala North (1963).

In a series of observations, the pattern was perforined in 63 out of 158 strikes $(40 \%)$. (Total of 200 minutes observations on 15 individuals). In 47 of 63 strikes preceded by neck-swaying, and in 85 of 95 strikes without neck-swaying, a prey animal was caught and swallowed. This shows that strikes were significantly less successful if preceded by neck-swaying (at level $P=0.05$ ). The pattern would not be retained if it was functionless, so it is possible that neck-swaying is used chiefly on prey animals which have already eluded the bird once, or on forms which the bird recognises as active and likely to escape. In this case the success of "strike only" relative to 


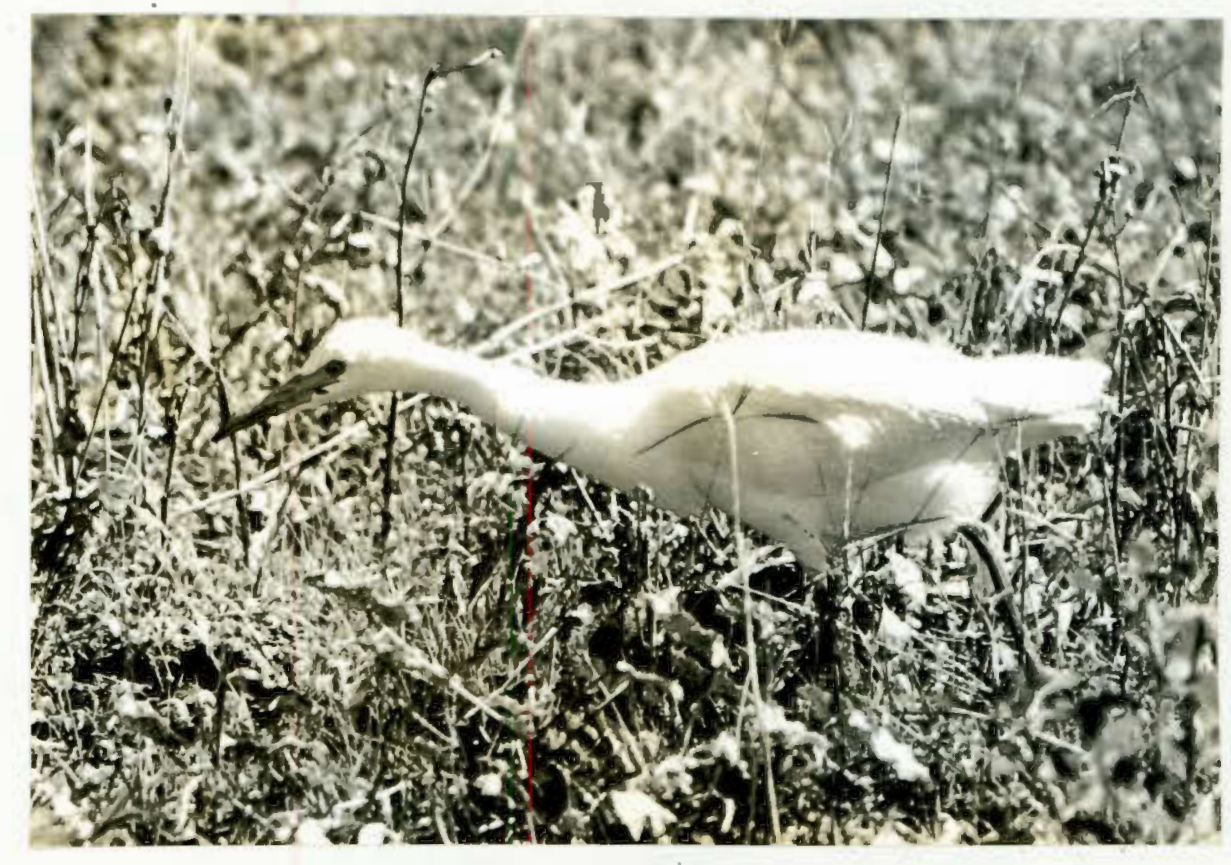

FIGURE 2. Stand and Wit position of a feeding bird. 
"sway and strike!" cannot be legimately compared. Slow neckswaying frequently occurs in birds slightly alarmed by the presence of a human, or in birds feeding in a small area hemmed in by buildings or trees. In both situations the movements accompany Alert, but it is unlikely that they serve any display function.

The author has only once noted the rapid bill-vibrating movements observed by Drinkwater (1958) in feeding Nycticorax nycticorax. A resting A. ibis walked to a small muddy pool, dipped the tip of its beak, and vibrated its mandibles rapidly for about two seconds. There was no animal life in the water so the behaviour was not functional, and is probably in fact so rare as to serve no purpose at all in the species; an animal does not only possess those structures and behaviour patterns which are adaptive, but also those which are not sufficiently detrimental to be selected against.

\subsection{Comfort Activities}

Motor patterns concerned with the care of the body surface have been included here purely for the sake of completeness, for as McKinney (1965) concludes, comfort activities are of little or no use in clarifying specific relationships. The present study is not sufficiently detailed to justify'motivational analyses of comfort activities. Only one of the activities listed below, preening, has acquired a signal value in A. ibis. Terminology has been standardised on McKinney's work on Anatidae. One point of interest is the large number (2l) of comfort activities listed in ducks, compared with the 10 in adult A. ibis, the difference being due to the fact that ducks have evolved a greater number of activities (particularly shaking) which serve to dry the body. Unless otherwise stated, the activities listed below do not differ from Meyerrieck's descriptions. The behaviour of young birds is dealt with in Section 6 .

Body-shake. The bird extends its neck, ruffles all contour feathers, rapidly shuffles its wings in and out a short distance from its body, then gives a rotary head-shake. The wing movements are clearly alternate and tail movements are absent. 
Wing-and-leg stretch. The leg and wing on one side are extended outwards, posteriorly and ventrally, the bird's weight being shifted on to the opposite foot. Almost identical to McKinney's (1965) illustrations.

Both-wing stretch. The head and neck are stretched forward and down while the wings, still folded at the carpal joint, are raised above the back. Very similar to McKinney's (1965) illustrations.

Jaw-stretch. The bird raises its head, opens its jaw and may make swallowing movements. In young birds, the movement is very common on awakening, suggesting that it is analogous to mamalian yawning, although this is not generally accepted. Jawstretch as described by McKinney does not occur in Ardeidae.

Scratching. The neck is partly extended and the foot is brought direct ("vornherum") to the head. Scratching is proIonged and vigorous ( 20 bouts averaged $16 \mathrm{sec.}$ ) and always performed with the pectinated claw of the second toe.

Bill-wiping. The slightly open beak is scraped up and down on the perch. No left-right alternations occur. Rare in A. ibis, this activity is common in Egretta garzetta, presumably because the latter feeds on fish and frogs.

oiling preening. The head is oiled by rubbing the "chin" and "face" on the exposed oil gland. Much commoner are the oil-distributing movements in which head and neck are vigorously rubbed and roliled over the back and area around the humemas.

Nibbling preening is the commonest comfort activity. No particular sequences were noticed although Meyerriecks details this. Particularly when longer feathers are being dealt with, nibbling is supplemented by a flicking movement in which the feather is rapidly drawn through the bill. Under-wing preening occurs in all three egret species and does not differ from Meyerriecks description and illustration. The only other family in which this activity has been recorded is the Ciconiidae, which suggests a close relationship between the two groups, as comfort movements are phylogenetically very conservative (e.g. Johnsgard 1965). Allopreening does not occur in A. ibis, although a pair-display, named here Back-biting and dealt with further in Section 3.5, has been incorrectly described by Harrison (1965) as mutual preening. Ritualised preening, probably a displacement activity, is described in section 3.8 . 
Bathing, On the single occasion on which bathing has been seen, an A. ibis standing in shallow water suddenly and briefly plunged its head under water, then gave 4 or 5 typical wingthrashing movements (McKinney 1965) before resuming immobility. This was repeated 4 times in 9 minutes, during which the bird was joined by another. A noticeable feature of the incident was the total absence of repeated head-dips, ruffling, preening and general excited movement which is characteristic of bathing in other families. Dust-bathing does not occur, which probably reflects the semi-aquatic origins of the family.

Gular Flutter. Fapid fluttering movements of the throat occur during hot weather. The beak is held slightly open and observations on young birds clearly show that the fluttering is due to movement of the hyoid. The activity is very common in nestlings which are still featherless. Sunbathing has not been seen in adult A. ibis, but occurs in adult Egretta garzetta and E. intermedius and in the young of all three species. Young A. ibis possess a number of behavioural temperature-regulating mechanisms, described in section 6.13 .

\subsection{Predation and Predator-responses}

Predation plays an unimportant part in A. ibis' biology. Only once in two years was any suggestion of predation on adult birds seen; an individual apparently killed and eaten by an owl. During the breeding season, both eggs and young are destroyed by predators, but the mortality incurred represents only a fraction of the total. Overall figures on mortality of eggs (17.6\%) and young are presented and discussed in Sections 5.6 and 6.2 . There is no way of determining the numbers of eggs and young destroyed by predators alone, but my impression was that the percentages were less than $10 \%$ in both cases. In addition, most chicks eaten by predators are less than 10 days' old, and as massive'mortality due to starvation occurs between the 6 th and 20th days (Section 6.22), the predators are in effect removing numbers of chicks which are due to die shortly. The overall effect of predation on breeding productivity in A. ibis is therefore slight. This is based on observation at a limited number of colonies, but predation may be far heavier in Central Africa, where there are greater numbers of raptors. Surprisingly, Teal's (1965) figures showed that predation in 4 other Ardeidae in one U.S.A. heronry was heavy: up to $40 \%$ of eggs and young in 
the smaller species. It is possible that, a selective effect of greator predator-pressure in Africa has produced relatively efficient predator-responses in A. ibis.

No indication of predation by mammals has been noticed, despite the fact that two species of Viverridae were present in the vicinity of the Paarl colony. In the case of animals which rely largely on olfaction to locate prey, the dirt and atink of a heronry must be an effective deterrent. Responses to predatory mammals have been observed in detail only in tame nestlings, and are therefore dealt with in Section 6.

On only one occasion has a raptor been seen to interfere with nesting A. ibis; an eagle (Stephanoaëtus coronatus) which spent 30 minutes in a colony, making numerous flights among the trees. Every time the eagle moved, most A. ibis nearby responded with an uproar of Kaaah calls (Section 3.94), although many birds remained Alert. Some adults and nestlings threatened the eagle with full-intensity Forward Displays, but there was no mobbing such as occurs in many Passerines for the birds never approached the eagle. At the same time few individuals flew away from the raptor. The great outburst of noise every time the eagle performed intention movements of flight probably has a distracting effect, for it left without any prey. Although. it cannot be proved that the eagle was hunting, it looked around continuously and made many short flights at perched A. ibis, which usually vacated their perches at the last moment.

The greatest single advantage of A. ibis' gregarious roosting and nesting habits probably lies in protection from predators, for gregariousness provides a very effective warningsystem and the above example suggests that responses by numbers of A. ibis may have a repelling effect on some predators. Feeding A. ibis are frequently startled by owls, smaller raptors and crows if they suddenly fly overhead, but the disturbance always dies down rapidly.

At the Paarl nesting colony the chief (and possibly only) predator was the Ardeid Nycticorax nycticorax. The total mortality due to predation was estimated to be less than $10 \%$ of eggs and young, but since there were only about 40 pairs of N. nycticorax breeding at the colony, as compared with 2800 to 3000 A. ibis nests, the former species had a considerable effect on the latter if their relative numbers are taken into account. In some areas, such as parts of the U.S.A., N. nycticorax is 
very abundant, and where it breeds in mixed colonies with A. ibis, could become an important factor limiting the latter species' spread.

Most A. ibis nests are never left unguarded from the start of incubation until the chicks are 12 to 19 days old. As A. ibis are not intimidated by $\mathrm{N}$. nycticorax and pay little attention to them unless within the individual distance (Hediger 1950), the latter species is only able to take eggs and chicks either when nest-owners are perched relaxed next to the nest, or have first been disturbed by humans. N. nycticorax have twice been seen to kill and swallow unguarded A. ibis chicks about 14 days old, whose parents had recently ceased to brood them. A N. nycticorax perched near a vulnerable A. ibis nest will suddenly dart to it, grab an egg or chick (sometimes knocking it out and losing $i t$ ) and fly off. Immediately it notices, the parent aggressively chases the fleeing predator, but never pursues it more than a few metres. On one such occasion, a number of A. ibie nearly gave loud Kaaah alarm-callas but apart from this no special predator-response by A. ibis towards N. nycticorax has been noticed. I obtained the impression that A. ibis is slightly more aggressive towards N. nycticorax than towards conspecifics, but apart from this it responds to the latter species as it does to any other Ardeid. Presumably these two species have not existed sympatrically long enough for more efficient predator-recognition to have evolved in A. ibis. A predator like N. nycticorax which is not recognised as such by its prey possesses a tremendous advantage, for the major problems of stalking and/or chasing are minimised.

Cott (1946), in a major study of visibility in relationship to edibility, rated A. ibis as one of the most conspicuous bird species of all ( 10 points out of 10 ), yet by a number of tests found it also to be highly palatable (19 points out of 20 ). This completely contradicts the normal inverse correlation between visibility and edibility, and A. ibis, together with one other species (Ciconia ciconia), form "outstanding exceptions to the general relationship". There is obviously no selection by predation in favour of cryptic coloration in A. ibis; so as a corollary, predation must have played a part in the evolution of A. ibis' behaviour, particularly its greparious roosting and nesting habits. It was not possible to test the adaptiveness in relation to predation of A. ibis' behaviour, as Tinbergen (in press) has done for Larus ridibundus, simply because predation 
was so infrequent. As most $A$. ibis nests are easily accessible to all birds of prey and smaller mammalian predators, and as the species does not have specialised "mobbing" responses, the deterrent effect on predators of prey numbers per se must be great. 


\section{DISPLAYS}

3.1 Introduction.

\subsection{Definitions}

By definition, displays are "those peculiarly standardised and often exaggerated performances, including all vocalisations and many movements and postures, which have become specialised and modified as social signals or releases" (Moynihan 1955). This process of specialisation is known as ritualisation. In practice, it is frequently a matter of personal opinion whether an activity acts as a social signal or not and in fact both Ficken (1966) and McKinney (1965) recognise this difficulty, the latter adopting arbitrary standards to distinguish between them. In addition there are no objective criteria for judging whether an activity is ritualised or not, for it is clear that displays exist at different stages of evolution, some highly ritualised and others very similar to the activities from which they are derived. Since there appears to be no way of escaping these problems the above definition, although not completely satisfactory, is retained and the word "display" will be used for all activities which are apparently ritualised.

There is a great deal of variation in the. use of the word "posture" in the bird behaviour literature. In addition, it implies a static attitude and since all A. ibis displays except two involve movement, the word "posture" is avoided here. This study follows the convention of listing displays as a series of distinct species - characters. While this system facilitates description it tends to obscure the large number of behaviour patterns which are not readily assignable to one display or another, a difficult point dealt with so far only by Moyninan (1966), and Dane et al (1959).

\subsection{Display analysis}

Following accepted practice, each display has first been described, then analysed in terms of:

(a) causation (and where possible the factors causing cessation as well);

(b) function;

(c) evolutionary origins.

In adition, section 6 includes points on 
(d) the development of some behaviour patterns.

The following system is based on Hinde (1955), Tinbergen (1959) and Baerends (1962).

(a) Causation. The exact external factors causing (i.e. releasing) a display should ideally be found by experiment. In an observational study, it is sufficient to describe the environment situations in which each display occurs.

Internal factors. Work on a number of species (e.g. in Andrew 1961, Hinde 1955, Marler 1956a, Morris 1956, Noynihan: 1966,) has shown that all displays are simultaneously motivated by two or more tendencies, which have been defined as "the readiness to show a particular type of behaviour, as observed under natural conditions" (Marler 1956a). By convention, the three "major" tendencies are fear, aggression and sex, with hunger, nest-building and parental tendencies of lesser importance. The relative strengths of these tendencies in a display may be assessed from three sources.

(i) The behaviour which is shown during and immediately before and after the display, e.g. witharawal indicates fear, attack indicates aggression.

(ii) The context in which the display occurs. E.g. a female attracted to an unmated male is assumed to be sexually motivated. (iii) Some display components form very useful indicators. For instance, A. ibis has an extremely wide range of crest positions (Section 3.2 and figure 3 ), which provided such highly sensitive tendency-indicators in agonistic situations that it was not necessary to consider any other components when estimating the balance between fear and aggression in a display.

(b) Function. Outward responses to a display are in some cases obvious. Hormonal effects, while equally important, cannot be investigated in an observational study. Remarks on the ultimate biological function of any particular display are to a large extent speculative.

(c) Origin. All displays are derived from simpler, unritualised activities. The origins of some display components, particularly those associated with threat, are obvious. The homologies of highly ritualised patterns are investigated by two methods. 
(i) The individual components of the display are compared with those of all possibly homolous activities in the species. The greater the similarity between the components of two displaya, the more closely these displays are assumed to be related.

(ii) If two activities have similar internal and external and external causal factors, they may be homologous.

3.13 Display frequency

Since 1900, A. ibis' range has expanded tremendously, the most striking aspect of the species' spread being its establishment in America, where it has occupied an estimated two million square kilometres since 1930. One aim of this study is to provide material for a comparison between the African and the American populations of A. ibig, for it is very likely that the courtship displays of the two populations will:eventually diverge. Dobzhansky (1951), Hinde (1959), Mayr (1963) and many others have recognised the importance of courtship displays a isolating mechanisms. Selection for specific' distinctiveness of isolating mechanisms must be particularly strong in colonial nesters which breed in immediate proximity to a number of related species.

It is widely accepted (e.g. Dobzhansky 195I) that selection for divergence follows sympatry. Since A. ibis breeds in company with a completely different set of species (excepting Nycticorax nycticorax) in African and American heronries, the requirements for specific distinctiveness of displays must differ in the two populations. In addition, several workers (Perdeck 1958, Blair 1955, Crook 1963, 1964) have stressed the role of ecological factors in bringing about display changes, and it is already known that variations of a species' behaviour in different parts of its range may occur in response to environmental factors (Curio 1961).

Following on this, the patterned movements of displays are to a large extent species-constant and stable, but their occurrence is influenced by the external situation and by hormonal states. Hence the frequency of occurrence of a display must be more plastic than its physical appearance, so will be the first feature of a display to be altered.

For this reason; most display descriptions are accompanied by figures on their incidence. The figures (table l) were all obtained from detailed time-scores for individuals whose sex and stage in the breeding cycle were known. Only time-scores 
for individuals which had been observed for at least 10 consecutive minutes were used, so they represent only a fraction of the total observation time, since the majority of notes on unmated and recently paired birds were fragmentary. In obtaining averages, care has been taken not to group different phases which might not be comparable.

\subsection{Agonistic Displays.}

Several displays, all part of a continuous series of behaviour patterns, are grouped under this heading. In all A. ibis agonistic digplays the crest-feathers provide accurate tendency-indicators, so will be dealt with in detail first.

Figure 3 illustrates 9 nodal points in the range of veriation of crest positions and abundant evidence (below) indicates that each position is produced by a different combination of fear and ageressive tendencies. Given any crest position, one can usually infer a bird's motivational state and predict the ensuing behaviour and effect on other: birds. The system does not apply when sexual tendencies are present and in "reversed" displays such as Stretch Figure 3 illustrates biras in breeding plumage; the crest feathers of non-breeding birds are shorter but their movements similar.

Position 1 is characteristic of birds which are totally relaxed and inactive, usually while on the nest site: fear and aggression very low, if present at all.

Position 2 is given prior to Forward Display, while walking towards a trespasser or when weakly threatening another individual: fear very low, aggression moderately hich.

Position 3 represents full-intensity threat, the bird advancing towards another and either repelling it or becoming involved in a fight: fear very low, aggression very high.

Position 4 is characteristic of resting birds which are aware of the nearby presence of a human, and also occurs in birds resting on the ground: fear moderate, aggression very low or absent.

Position 5 is characteristic of what can best be termed "curiosity"; unmated females peering at unmated males (described in section 5.31); nestings staring at an unfamiliar object or potential source of danger: all situations presumably representing approach-retreat conflict with moderate fear and 
FIGURE 3: Crest positions:

explanation in text.
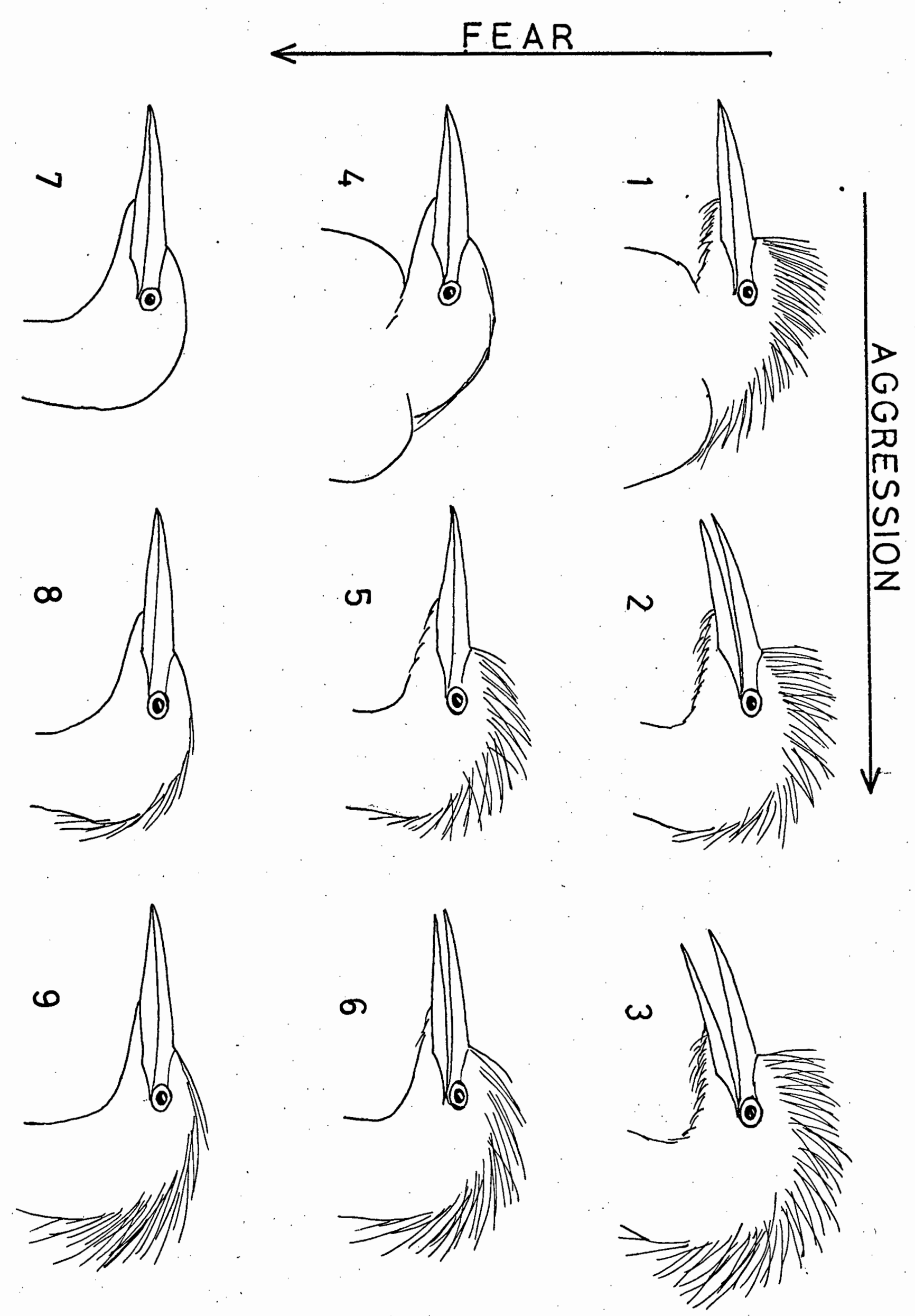
FIGURE 4 .

Distribution of scalp feathers of an adult male A. ibis. Each circle represents a single follicle. Figures denote total number of follicles per $5 \mathrm{rm} \cdot \mathrm{m} . \mathrm{X} 10 \mathrm{~m} \cdot \mathrm{m}$. rectangle.

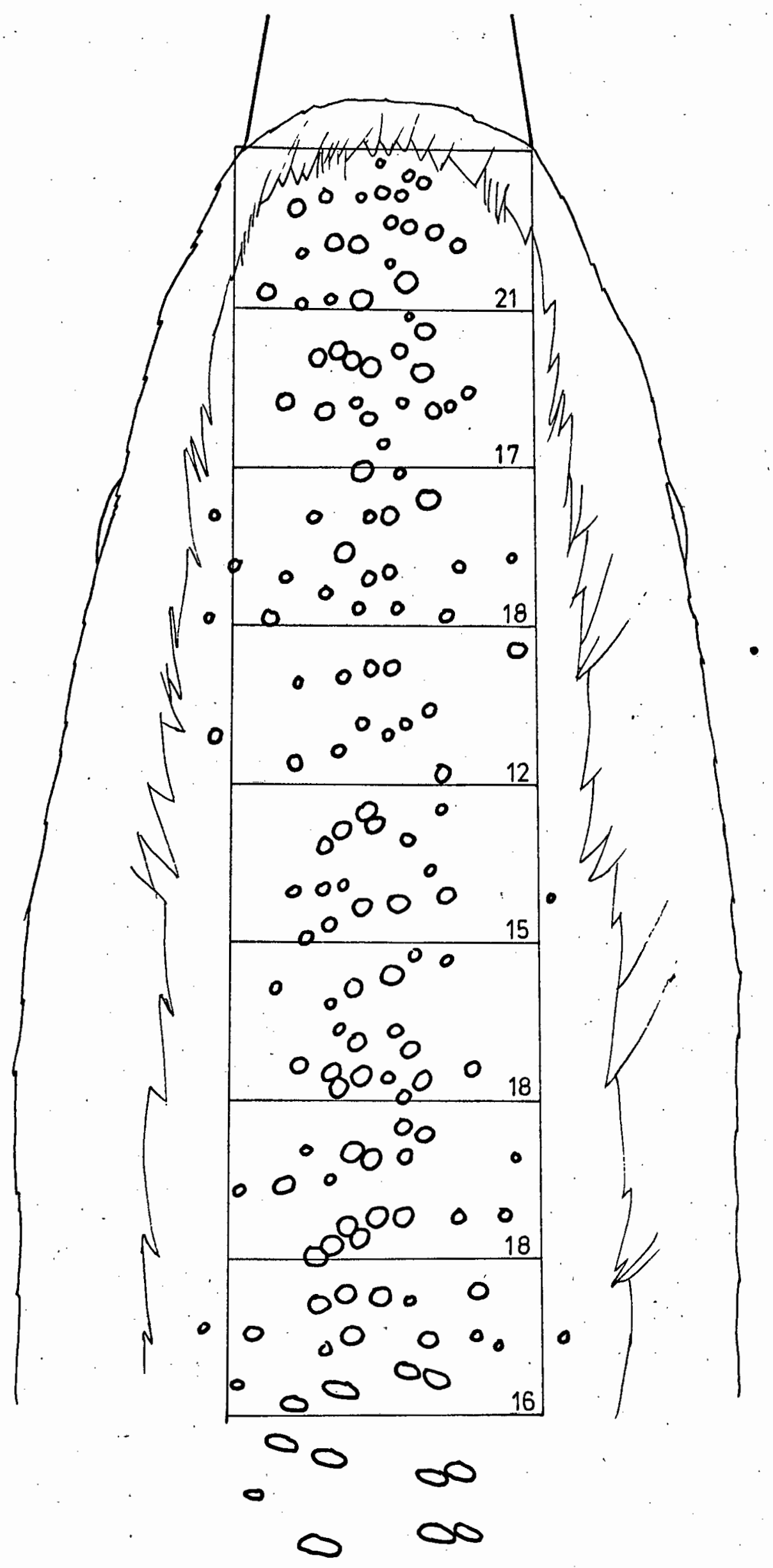


aggressive tendencies.

Position 6 is characteristic of territory-defending birds which have just threatened in return: fear moderate, aggression high.

Position 7 occurs in birds highly conscious of an approachhuman or predator and usually precedes flight: fear high, aggression low or absent.

Position 8 occurs in birds which are remaining on their sites despite the proximity of a predator, and in non territoryholding birds which remain on a perch despite high-intensity threat by another bird: fear high, aggression moderate.

Position 9 is characteristic of birds involved in highintensity mutual threatening causing balanced conflicts in which neither bird retreats or advances for several seconds: fear and aggression high.

It is clear from the illustration that the pilomotor muscles of the anterior and posterior regions of the crest are differently motivated, with erection of the anterior feathers inhibited by fear tendencies and erection of the posterior crest caused by moderate to high aggressive tendencies. Fear tendencies have little effect on the posterior crest and aggressive tendencies have little effect on the anterior erest. This is similar to the situation in the Stickleback Gasterosteus where erection of the dorsal spines is associated with attacking and erection of the ventral spine with the fleeing (van Iersel 1953). The dichotomous nature of the crest movements are reflected to some extent in the anterior-posterior distribution of the scalp feathers, shown in figure 4. Meyerriecks states that "there is great variation in crest-elevation" but did not notice any functional and motivational differences between the anterior and posterior regions.

Most agonistic displays are orientated towards another individual and this is a major cause of variation, for the form of a display depends on. whether the other bird is on a higher or lower plane, near or far, on its nest or not. Selection for "typical intensity" or display distinctness in A. ibis' agonistic displays: appears not to have been strong, possibly because the species is not highly aggressive and fighting is uncommon.

Figure 5 illustrates 6 skeletal attitudes in agonistic displays. For the reasons stated above, any separation of 
FIGURE 5: Agonistic displays.

$$
\begin{gathered}
\text { A - C Forward Displays } \\
\text { D Withdrawn Crouch } \\
\text { E Supplanting Run } \\
\text { F Alert }
\end{gathered}
$$
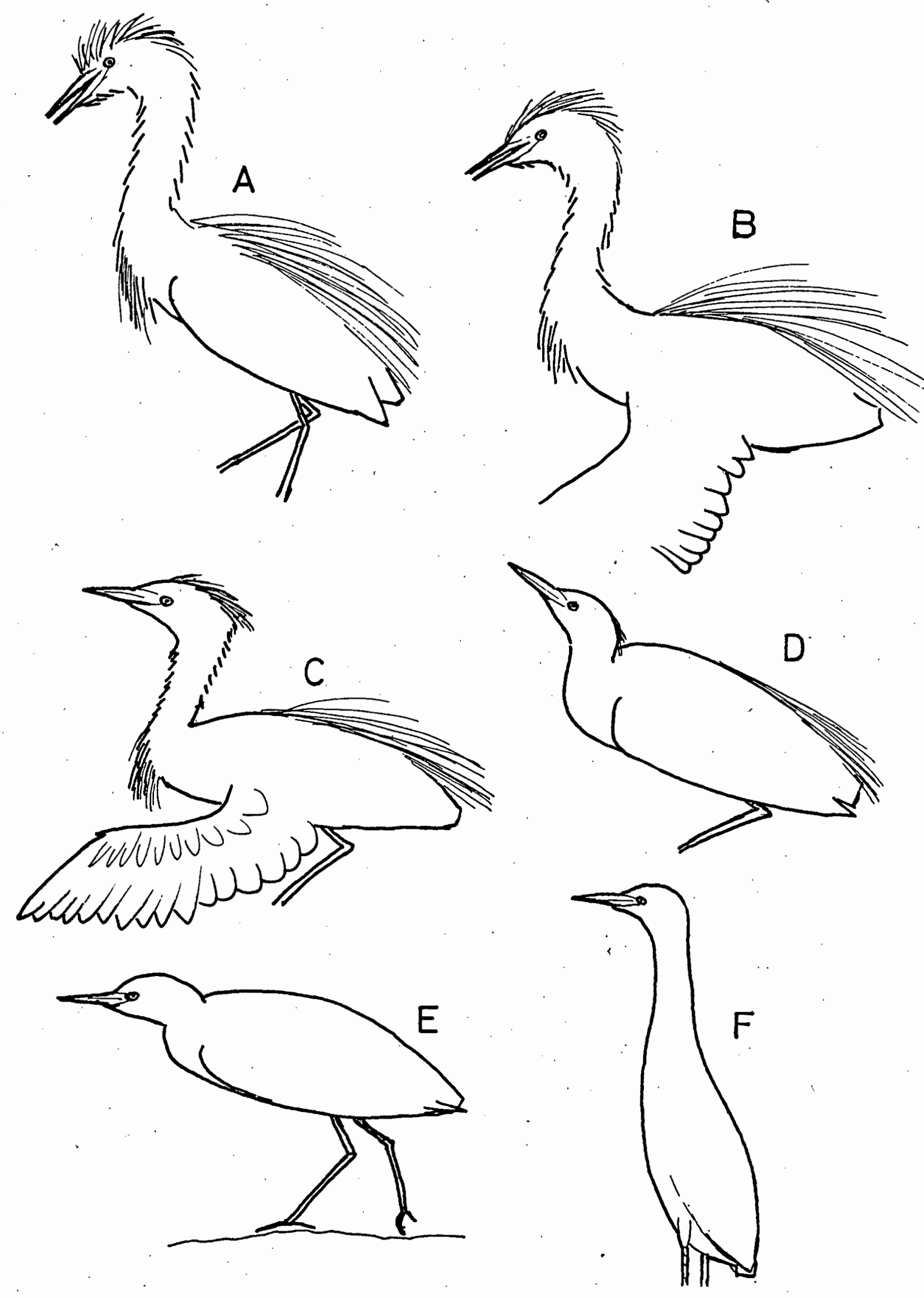
these displays must be arbitrary, but four are distinguished here, chiefly in order to simplify description.

\subsection{Forwerd Display}

Forward Display includes a wide range of skeletal and feather attitudes, all characterised by the fact that they are always directed at another. individual, and have a distanceincreasing effect (Tinbergen 1959). Iittle attention has been paid to Meyerrieck's inconsistent system of agonistic display naming; (for example both figures 8 and 37 (in Meyerriecks) are labelled "Forward Display", yet the illustration in figure 37 is obviously far more similar to figure 35, which is labelled "Aggressive Upright Display", than it is to figure 8).

Forward Display in A. ibis contains the following components:-

1. Crest positions $2,3,6$ or 9 .

2. Neck feathers, pectoral and scapular plumes partly to be fully erected. Sometimes the crest and scapulars are under the same neural control, for on occasions all their up-down movements are exactly synchronous.

3. Neck may be partly retracted into the shoulders (figure 5, position $c$ ), extended anterior and upwards in, an S (position A) or posteriorly of this (position $b$ ). (The more retracted and posterior neck indicate higher fear tendencies.)

4. The beak is directed slightly downwards and opened at higher intensities.

5. The bird may slowly pace stiff-legged towards its opponent (crest position 2 or 3 ).

6. The bird stabs at its opponent with the neck and beak ( $86 \%$ of 116 cases). Contact was made in only $4 \%$ of observed cases, but probably occurs more frequently in unmated males, which sometimes fight over territories. In Forward Displays between birds on adjacent nests (usually crest positions 6 or 9), the birds very rapidly lunge at each other a few (2 to 11 ) times, each bird drawing back as the other stabs and vice versa (figure 6). This is not overt attack but a highly ritualised ceremony, for in very many cases the birds are capable of striking each other if they stabbed simultaneously, but they do not. Skead (1966) also noticed this. (The distance between A. ibig broodpatch, which is in the centre of the nest, and the beak tip is 


$$
\operatorname{lig}^{2}
$$


FIGURE 7. The wing-beat component of Forward Display.

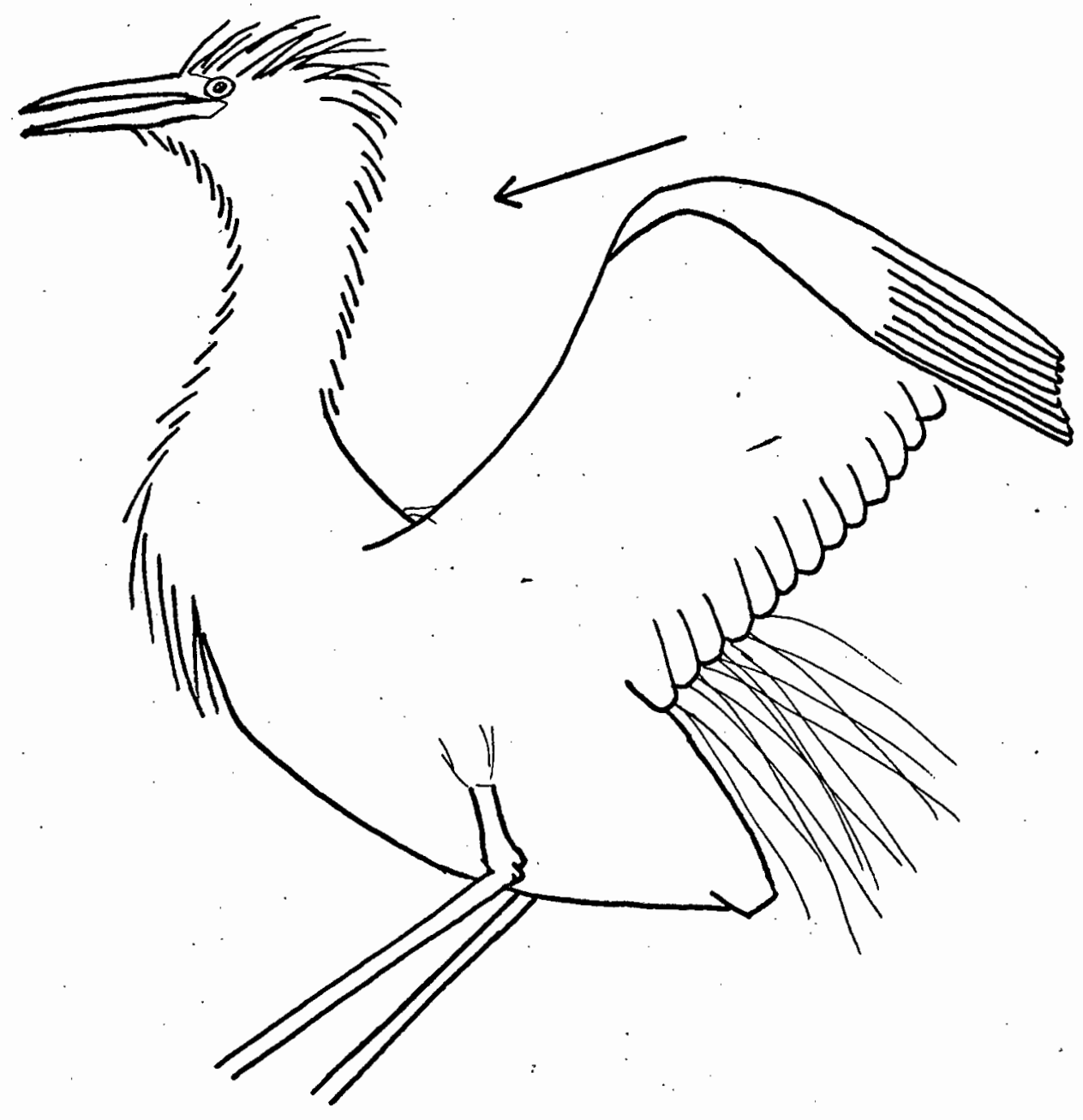


$3^{\circ} 0$ to $40 \mathrm{~cm}$. Many nests have their centres less than $2 \times 30 \mathrm{~cm}$. from their nearest neighbour's, yet only once in many hundreds of observations has one sitting A. ibis been observed to strike another). This ritualised stab-and-counterstab only occurs intraspecifically, for A. ibis and Phalacrocorax africanus on adjacent nests have no such inhibitions towards each other and frequently peck and bite each other's heads.

7. Simultaneously with the stab, the bird gives a brief, harsh "raa" call. During the period when the bird's soft parts are red, (section 5.1) this is replaced by a muffled, gulping "rolk" call, ("thonk", Skead 1966), the:functional significance of which is discussed in section 5.31 .

8. Simultaneously, the wings are partly spread and one forward and downward beat made ( $48 \%$ of 116 cases); (figure 7 ). The wines may be held extended for several seconds. Usually associated with crest position 3 .

9. In a minority of cases, the bird supplants its opponent by flying directly towards it (crest positions 3 or 6). Aerial fighting rarely occurs; one bird flies directly at another which flies up to meet it, the two colliding in mid air; very sinilar in appearance to fighting in Leucophoyx thula (Meyerriecks).

10. Very rarely, but particularly during extra-pair copulation (Section 5.92), two birds will fight in bodily contact with many pecks and wing-beats.

Figure 8 gives the frequency of changes in Forward Display which occur during the course of nesting. Mutual stab-andcounterstab encounters (above) were recorded as single displays by each bird. The histogram shows that males are most aggressive before egg-laying and during the early stages of incubation but that as incubation proceeds their Forward Display frequency declines, rising slightly again after the eggs hatch. Females give relatively few Forward Displays during pair-formation but becone slightly more aggressive towards other birds as incubation proceeds, and from the second half of incubation onwards are more aggressive than their mates. The sharp rise in display frequency after the eggs hatch must reflect a rise in parental tendencies, for there is no corresponding change in the daily attentive pattern (section 5.83). Increased aggressiveness after efg-laying is also found in Kaaah calls.

Although direct comparisions are not possible, A. ibis 


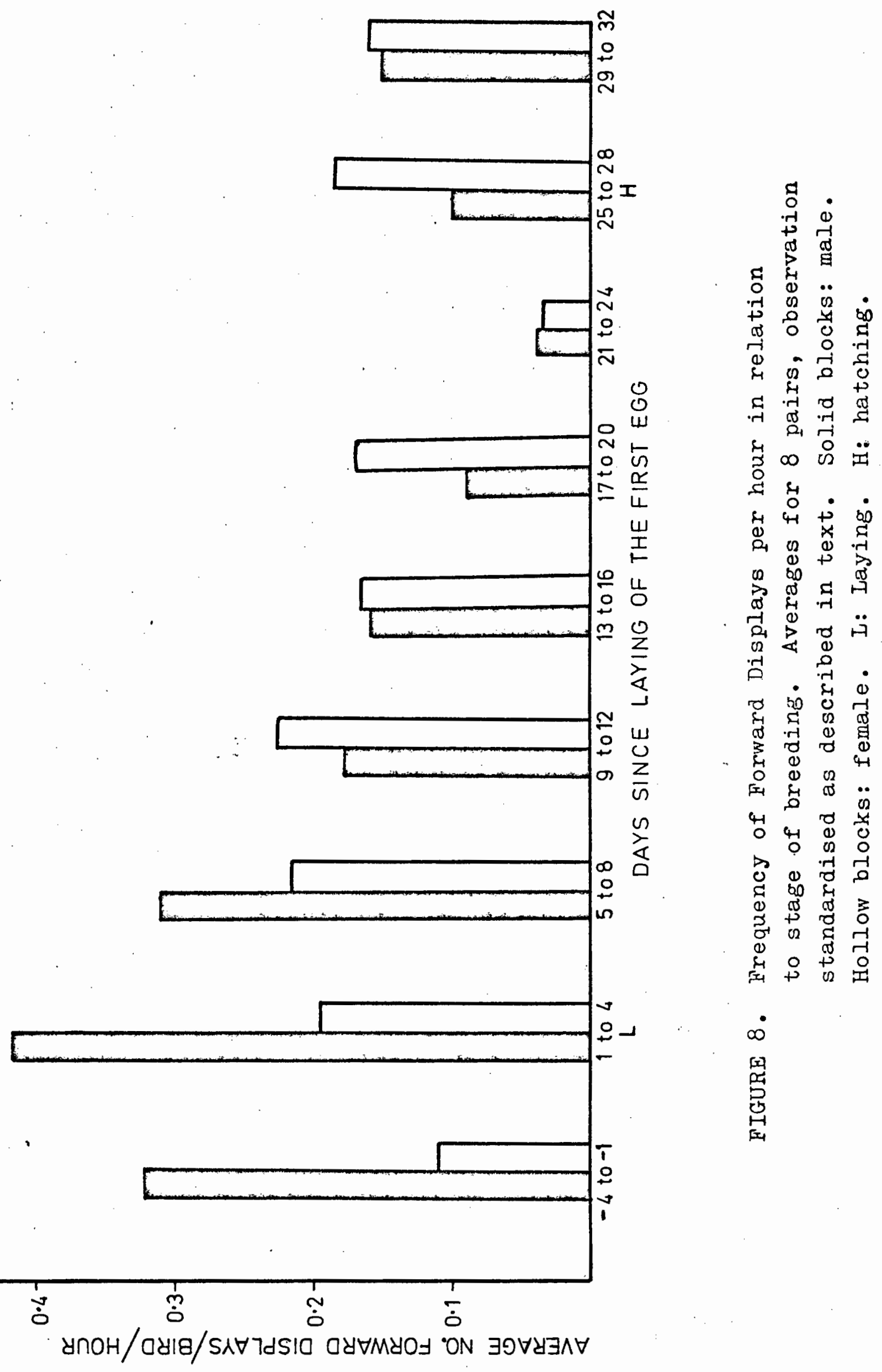




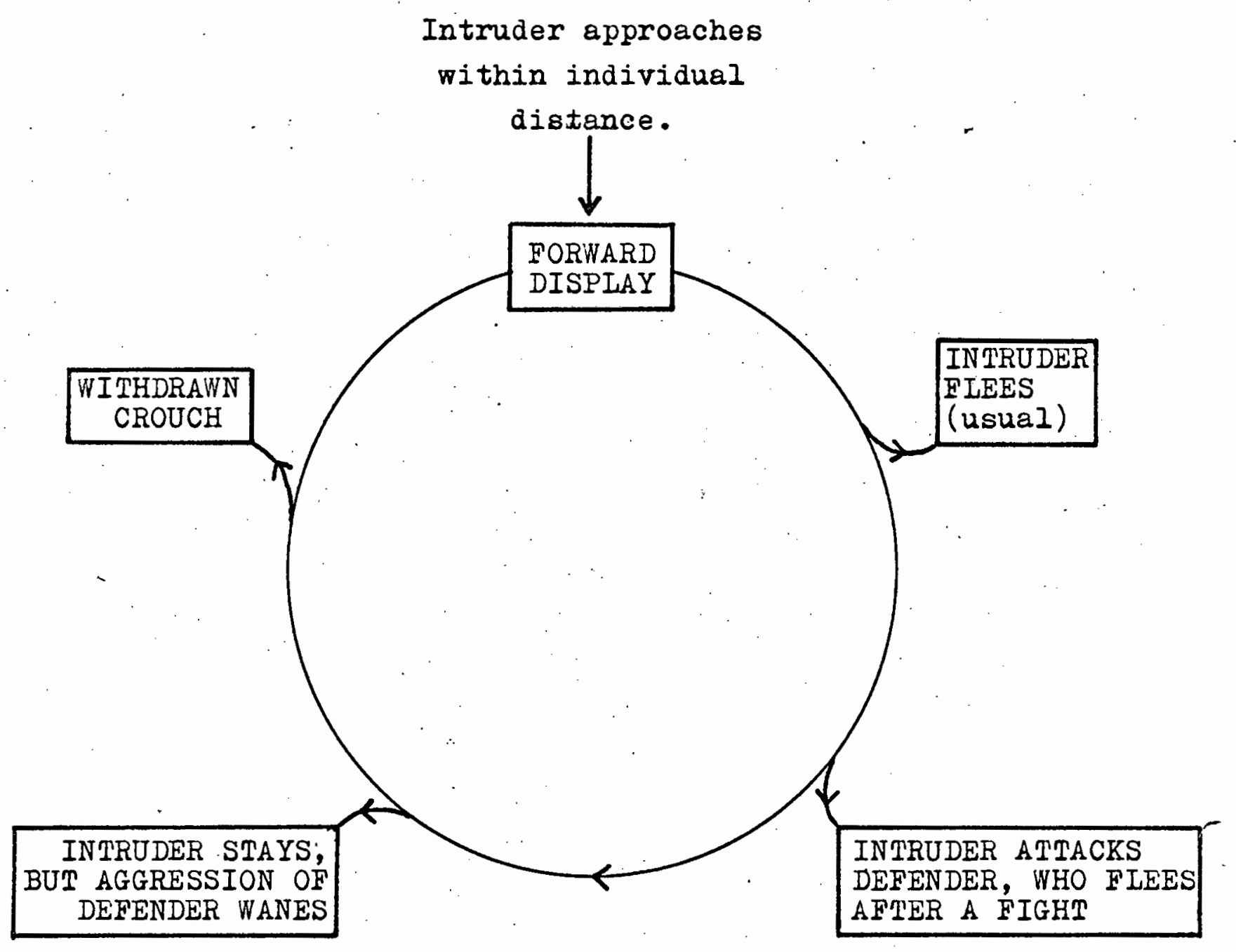

FIGURE 9. Diagram of the relationship between Forward Display and the four chief factors which "switch it off". 
appears to threaten considerably less frequently than do other colonial nesters for which figures are available, such as Sula bassana, which threatens approximately five times as frequently as A.ibis does (Nelson 1965).

Cause. As inferred from the crest positions, the display is characterised by high aggressive tendencies in conflict with fear tendencies of varying strength. The usual external releaser is violation of the bird's individual distance, (Hediger 1950), whether it is defending a nest, a territory prior to mating, or a perch at a communal roost. The incidence of Forward Displays is affected by a number of social factors discussed in sections 5.3 and 5.9, where they have greater relevance. One point of interest is that aggression between paired birds only occurs in exceptional circumstances.

This is one of the few displays where the factors causing cessation are readily apparent. In figure 9 the relationships between the chief releaser and the four chief "switch off" factors are illustrated diaframatically. The point to note is $r$ that appeasement or submissive displays which are admittedly infrequent, rarely "switch off" aggression in an agonistic encounter, and that aggression normally only ceases when the distance between two reacting birds is increased. On one occasion, a male A. ibis threatened a nearby Egretta intermedia with about 18 high-intensity Forward displays in rapid succession ( 5 in succession was the most that had previously been observed in any similar situation), but did not actually approach it. The larger Egretta did not retreat or show any response at all, as an A. ibis would have done, which neatly demonstrates that Forward Displays are normally only "switched off" when one bird retreats.

Situations related to the above can frequently be seen between adult and young A. ibis. Adults almost invariably retreat when threatened by nest-defending chicks, even though they could easily overcome the chicks in a fight. Both the above examples illustrate strikingly that the appropriate response to a display is just as highly ritualised, and just as important in the species' social organisation, as the actual display components are themselves.

Overt fear in a bird (crest position 7), as opposed to its actual retreat, does not inhibit aggression, for on several occasions a bird has been seen to catch a leg or wing in a 
branch while escaping from an asgressive bird which continued to attack and peck it. Head-flick (section 3.7) is of uncertain signigicance as an inhibitor of agoression.

Function. Forward Display is a threat display which serves to maintain individual distances and to prevent nest sites from becoming indefinitely compressible. All feather components serve to increase the bird's apparent size, and most other components $(3,4,5,6,8$ and 9) are intention-movements of:attack (below) which combine to provide unambiguous signals of aggressiveness. As elaborated above, a bird's crest positions usually enable one to predict its behaviour over the ensuing few seconds. If this is the case for human observers, then within the species the crest must function as a highly sensitive tendency-indicator, enabling one bird readily to recognise another's intentions, thus helping prevent.unnecessary conflicts.

Noble and Wurm (1942) found that the lanceolate head plumes of Nycticorax nycticorax serve to strengthen the pair bond, as removal of the male's plumes caused the female to become less responsive. Crest erection in A. ibis has a distance-increasing effect, which illustrates the dangers inherent in assuming that homologous structures have similar functions.

Origin. Most of the skeletal movements of Forward Display can be interpreted as intention movements of packing at or walking or flying towards another bird. The S-shaped neck is a preparatory position for pecking, as is the downward-pointing beak, while the wing-beat component is an intention movement of flight. The last-mentioned is the only skeletal component which is not a functional prerequisite for pecking, so must be the most highly ritualised component.

\subsection{Supplanting Run}

This display is performed only on the ground by resting or feeding birds, where it replaces Forward Display functionally. With neck retracted, body axis about $30^{\circ}$ above the horizontal, and with crest positions 4 to 2 (figure 3 ), the displaying bird runs at another with stiff-legged strides (figure 5, position e). Supplanting runs usually occur when two birds compete for individual food items, with one bird chasing another for one or two metres, and have also been observed in $20-30$ day nestlings on the ground. The display also occurs in 


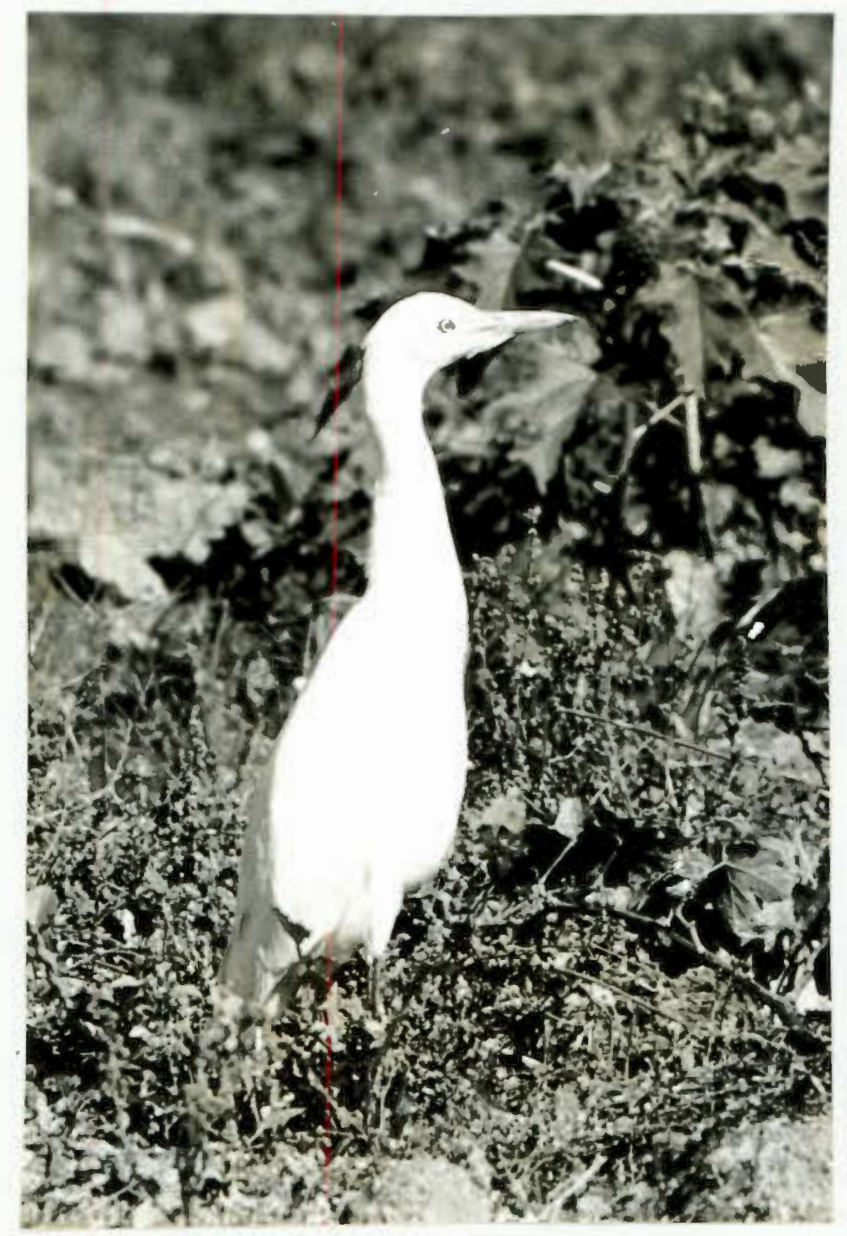

FIGURE 10. Alert. 
gatherings of birds which sometimes occur at midday, when some newly-arrived birds are supplanted in the above manner. However, in all the above contexts the display is unusual, so it plays a minor part in the species! biology; for this reason the degree of ritualisation appears low, with the display little removed from low-intensity overt attack. If a supplanting run does not cause the opponent to retreat, both birds usually fly at each other and clash briefly, giving low-intensity "raa" calls. The incident which Almond (1955) describes was presumably one of these.

\subsection{Witharawn Crouch}

This is very similar in appearance to the display of the same name which Meyerriecks describes in Butorides virescens. The bird suddenly crouches (figure 5, position d), with body horizontal, fully flexed legs, slightly raised scapulars, neck retracted and crest positions 7,8 , or 9 . Withdrawn Crouch is only given in response to Forward Display by another bird immediately above it. The crest positions indicated high fear tendencies in conflict with low to high aggressive tendencies. The display probably functions as an attack-inbibitor but occurs so infrequently that it must be of minor importance in this respect. Several components of Withdrawn Crouch are very similar to the downward movement of Stretch (section 3.3): flexed legs; retracted neck, beak pointing up and crest erect so it is very likely that the display serves as a "reversed movement" of Forward Display as Stretch does. A full discussion of the subject appears in section 3.3 .

\subsection{Alert}

Alert represents one end of the spectrum of agonistic displays. It is characterised by upright stance, erect neck; fully sleeked plumage and bill tilted above the horizontal (figure 5, position $f$, and figure 10). It is elicited by the presence of humans or predators, is very common in feeding birds, and seldom occurs in interspecific encounters. The crest position indicates high fear and very low aggressive tendencies. Birds will often respond to Alert in one of their numbers by following suit, so that the attitude acts as an alarm signal. It also has the obvious non-signal function of making the bird taller, thus enabling it to see further. 


\subsection{Stretch Display}

Stretch Display occurs in unmated males, is absent in unmated females, and is performed by both sexes for the duration of the pair bond. Typically, Stretch Display contains the following components, illustrated in figure 11.

1. The bird stretches head and neck vertically upwards, with legs about one-quarter flexed and body at an angle of about $45^{\circ}$ to horizontal (positions $1 a, 2 a, 3 a$ in figure 11 ).

2. Crest is fully depressed, scapulars partly depressed.

3. After half a second or less in this position, the bird suddenly retracts its neck slightly and flexes its legs, so that the body is tilted forwards and downwards in one smooth movement into positions $2 \mathrm{~b}, 2 \mathrm{~b}$ or $3 \mathrm{~b}$. Simultaneously, the scapular plumes are fully raised. The beak remains at an angle above the horizontal.

4. The call which accompanies the display is variable. Unmated males typically give one ir more soft "ow" notes at the apex of the stretch movement, each note being accompanied by a slight downward bob, then a soft crooning "rooo" call on the main downward movement. Either the "ow" or the "rooo" notes may be weak or absent and $37 \%$ of 27 Stretch Displays in unmated males were inaudible.

Females seldom perform the fully intensity. Stretch described above. Normaily, a paired female performs a brief downwardsquatting movement with neck retracted (position $3 a$ and $b$, in figure 1I), head and beak pointing upward, crest flattened, scapulars raised, and rarely with a "rooo" call. For the duration of the pair bond, Stretch components are present in greeting ceremonies, described in detail in section 3.01 . Once again, the components of squatting, upward pointing beak, flattened crest and raised scapular plumes are present. Approximately at the stage when the birds' beaks fade from oringe to yellow, the call accompanying stretch gradually changes from the soft "ow - rooo" to a harsh "aah", similar in tone to the harsh "raa" of Forward Display but more drawn out and occurring a quite different situation.

The displaying bird seldom re-orientates its body towards its mate as Meyerriecks found to be the case in Butorides virescens. In A. ibis, Stretch never contains swaying components as 

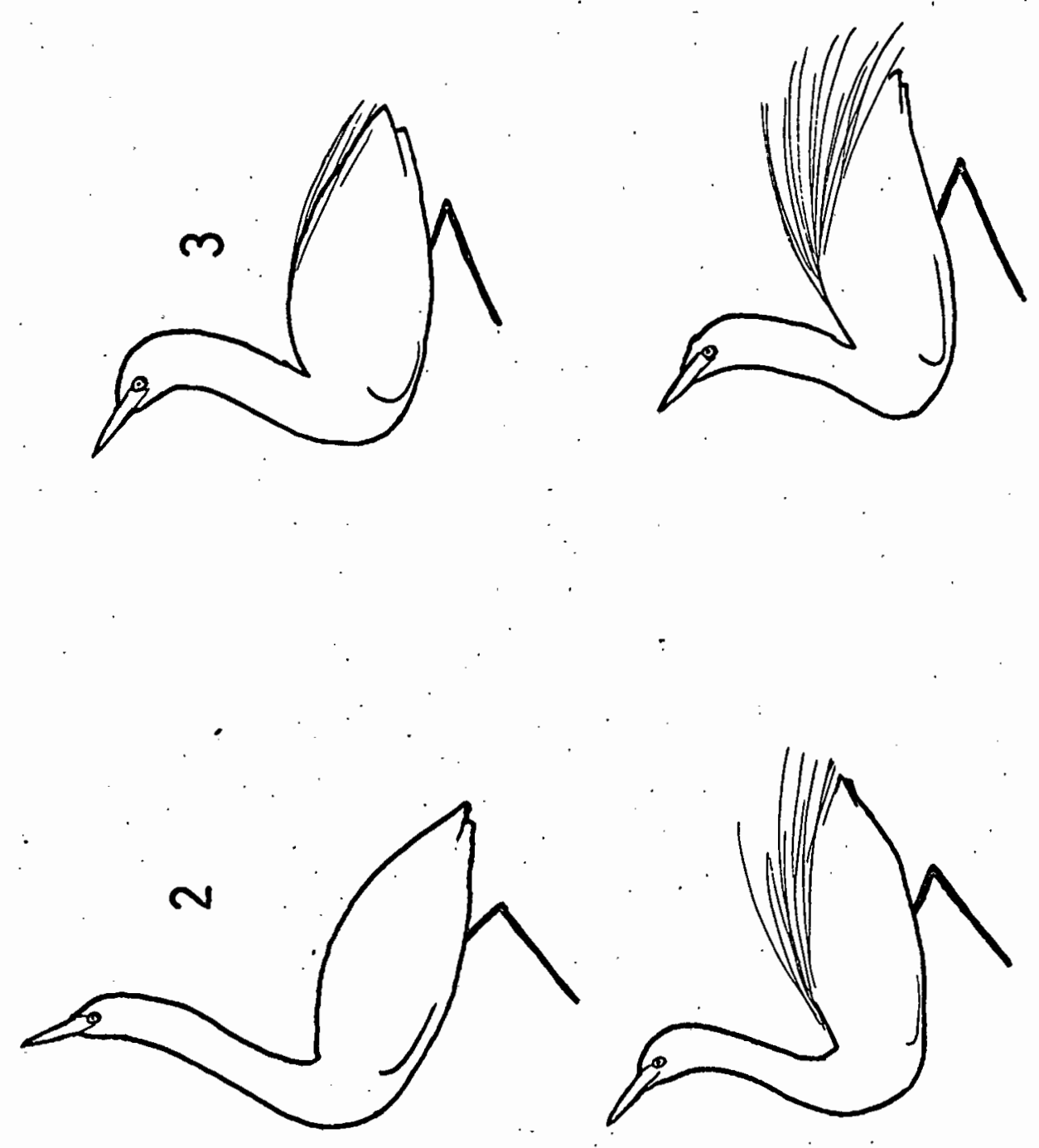

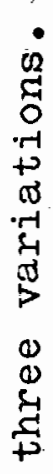
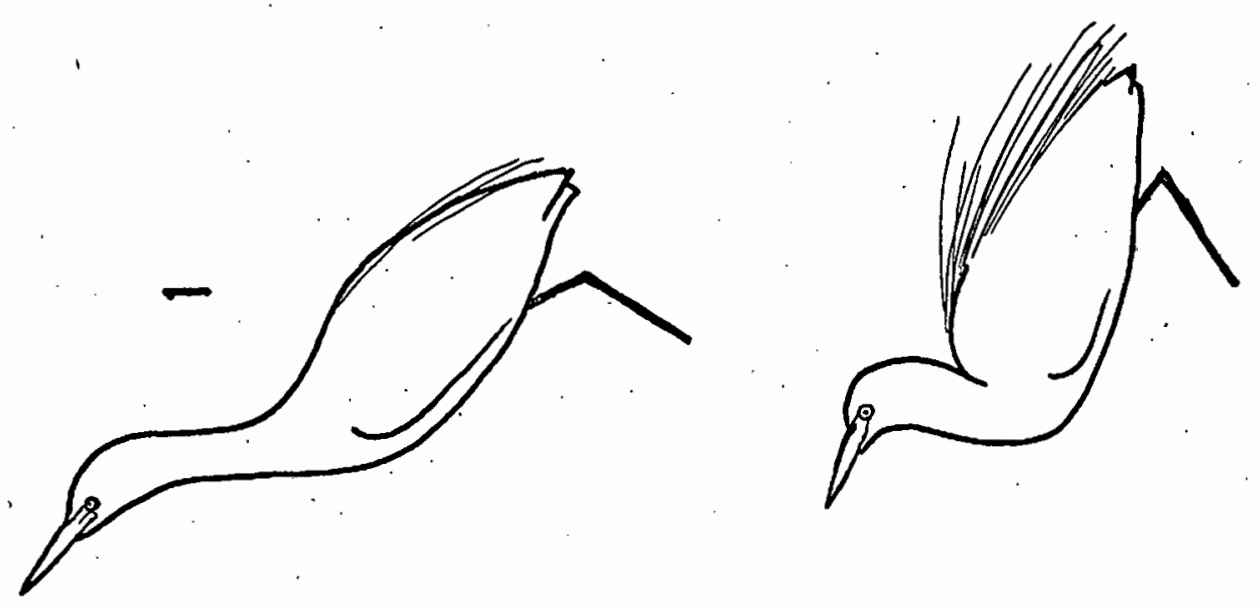

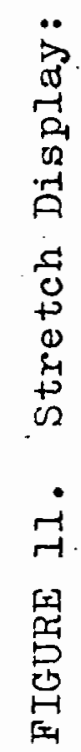

$\varangle$ 
in B. viresceus, although the swaying movements which are very common in unmated male A. ibis (section 5.31) may be homologous. The displays' frequency is hichly variable, so the figures given in table $I$ are of doubtful significance. In paired birds female stretch far more frequently than males do.

Cause. The majority (67\% of 49 in females, all 3 in males) of recorded Stretch Displays in paired birds are released by the mate's approach, whether it had been perched only one metre distant, or returning to the nest after a prolonged absence. The two situations are so similar that they have not been treated separately. In contrast to paired birds, the majority of Stretches in unmated males seem spontaneous and are not given in response to any environmental change apparent to the observer. In addition, table 1 shows that the display is far more frequent when there are no unmated females nearby, situations which are characterised by relative inactivity on the part of males.

Stretch is never accompanied by advance or withdrawal on the part of the displaying bird, and the display is never elicited by threat by the male or any other bird so fear and aggressiveness tendencies are presumably low or absent. If agonistic tendencies play a minor role, then it is axiomatic that sexual tendencies are present. This is supported by evidence in section 5.4, for stretch by the female is frequently immediately followed by copulation and (apart from Rickrack), Stretch is the only display which ever occurs immediately before copulation.

Function. Since it is inconspicuous in comparison with other common displays in unmated males (Chatter, Wingspread, Flap Flight Display), Stretch cannot have an important advertising function. In paired females, it often acts as a soliciting display leading to copulation, as shown by the figures and description in section 5.4. On these grounds, Stretch can be classified as the chief distance-reducing display (Tinbergen 1959) in A. ibis.

Several authors (Darwin 1872, Hinde 1952, Morris 1954, Marler 1956) have mentioned that displays which contrast greatly in appearance usually encode completely different signals. Stretch and Forward Display in A. ibis provide a classical illustration of this principle; the former is sexual and 


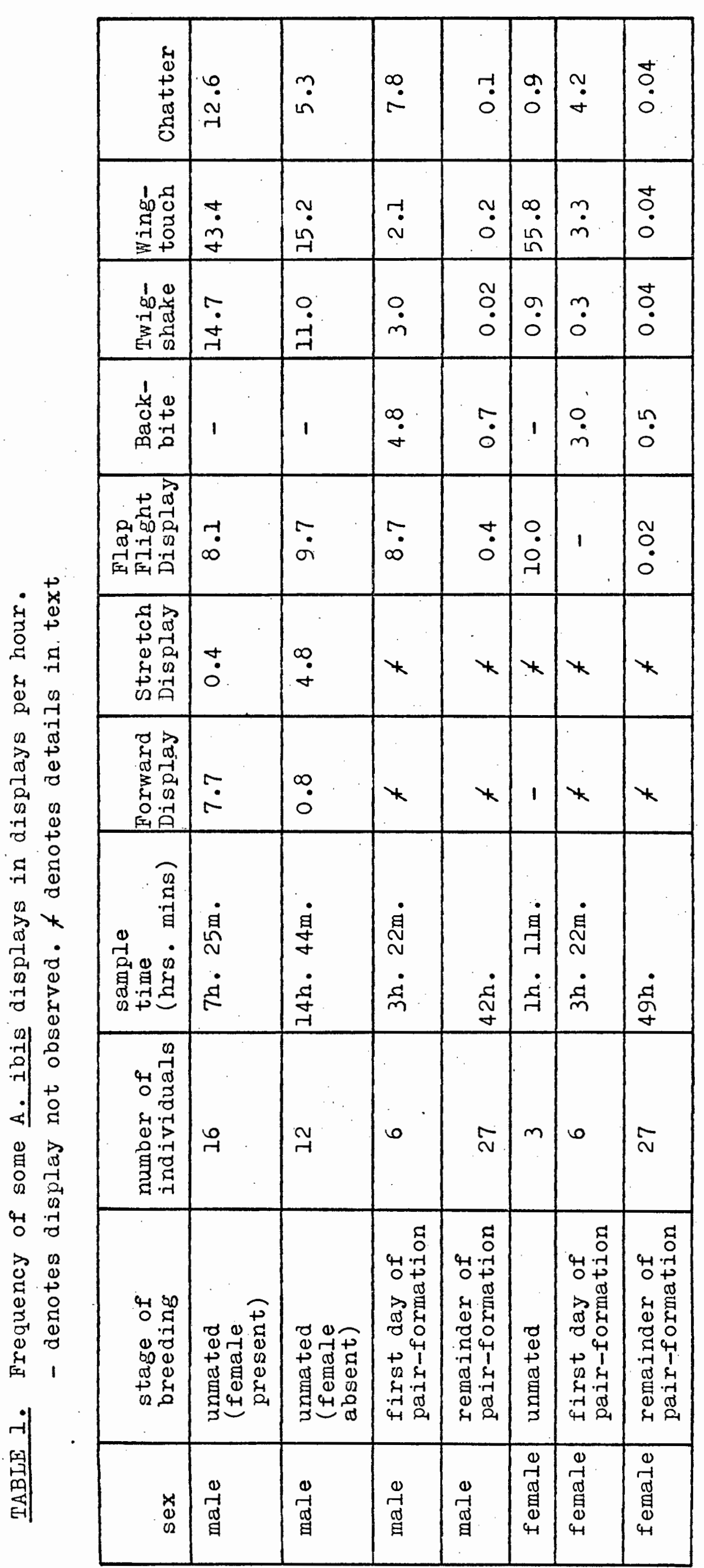


TABIE 2. Comparison of the components of stretch and Forward Displays

\section{Forward Display}

1. crest partly to fully raised

2. head and beak point downwards

3. loud, harsh call

4. scapular and pectoral plumes raised

5. neck feathers raised

6. forward lunging head and neck movements

7. displays always idirected towards another bird

8. bird approaches opponent

9. wings spread

\section{Stretch Display}

crest fully

depressed

head and beak

point upwards

soft, quiet call

scapular and pectoral

plumes raised

neck feathers flattened

head and neck first

extended vertically, then

retracted

no marked orientation in relation to another bird

bird crouches

wings closed 
distance-reducing, the latter is overtly aggressive and distanceincreasing. (An exception which proves the rule is discussed in section 5.31). Table 2 compares individual components of the two displays, showing that in almost every case the attitude or movement of one display is the complete opposite of the corresponding component of the other. Stretch is therefore said to consist of reversed movements of Forward Display (e.g. Marler 1956).

Origin. Stretch Displays, all clearly homologous to one another, have been described in all Ardeidae which have been studied in detail so far; Ardea cinerea (Verwey 1930), Butorides virescens, Dichromanassa rufescens, Egretta thula, Ardea herodias (Neyerriecks), Florida caerulea (Meanley 1955), Ardeola ibis, Egretta garzetta, E. intermedia, (this study), Ardea purpurea, (pers. obs.), A. melanocephala (North 1963). It is therefore safe to assume that the display occurs in all Ardeidae. If Stretch is universal in the Ardeidae, it must have evolved very early in the families' history. (Neyerriecks describes stationary and Extended Stretch Displays but, in Ardeola ibis at least, these forms just represent points in the display's range of variation, so separation is not justified.)

Daanje (195I), referring specifically to Ardea cinerea, states that Stretch is derived from the intention movements of takeoff. This is supported by slow motion films taken of Ardeola ibis. The downward-crouching phase ("bow") of a takeoff sequence (figure I) is similar to the crouching movement of Stretch in many respects; strongly flexed legs, forepart of the body tilted downwards, head and neck partly retracted. Daanje's explanation is therefore almost certainly correct, but deals only with the downward-crouchins movement of stretch and not with the more characteristic upward-stretching movement. The behaviour of Egretta garzetta and E. intermedia (section 7) during greeting ceremonies indicates the origin of the upwardstretching movement(s) of Stretch. In both these species, the bird on the nest never performs a Stretch. Display as in A. ibis, but stands and extends its neck up towards its mate, with bill and head pointing upwards, always re-orientating to face its arriving mate. It is quite clear that these neck-extended and head-up movements give the bird binocular vision of its mate. In addition, identical movements were frequentiy noticed in tame young A. ibis when they stared intently at some novel 

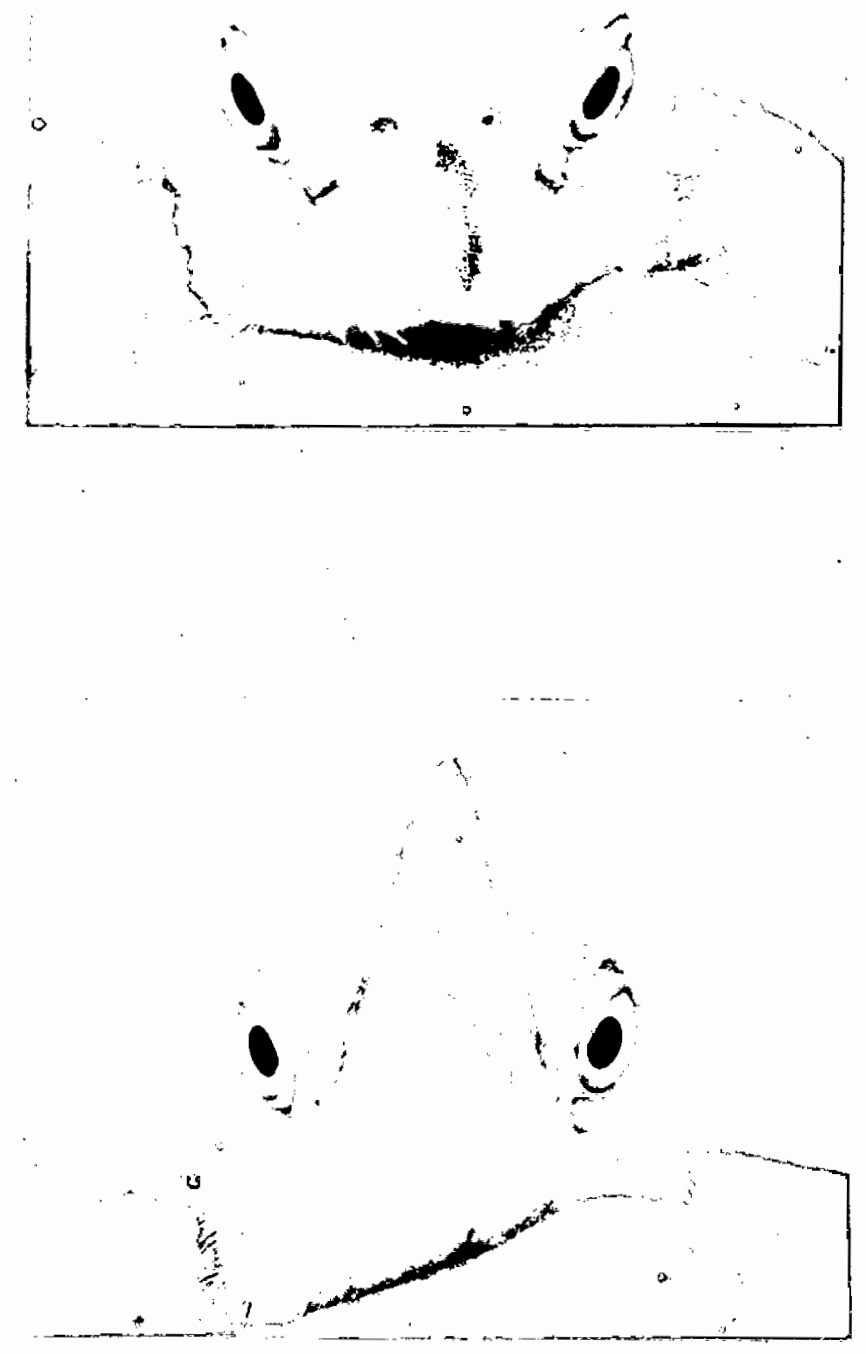

FIGURE 12. Illustrations of the ventral positions of the eyes and the field of binocular vision in $\mathrm{A}$. ibis 
object or source of disturbance.

The eyes of all Ardeidae are situated slightly ventrally and consequently, as figure 12 shows, the field of binocular vision lies below a horizontal plane passing through the head. It is therefore likely that the beak-up and upward-stretching components originally evolved as movements whereby the bird watched its mate closely. The movements of greeting still serve this purpose in Egretta, but in A. ibis Stretch is seldom orientated towards its mate.

The present author is in full agreement with Daanje's (195I) opinion that the downward-crouch of stretch is derived from the intention movements of flight. "It is concluded that the "bow" of takeoff became ritualised because it provided an unambiguous signal of non-aggression in situations where this is required (greeting and copulation). Additional components such as the flattened crest and soft call evolved for the same reason. To summarise, Stretch probably originated as the attitude necessary for binocular vision, immediately followed by appeasement crouching, derived from the intention movements of flight. These same principles can be used to explain a display in young birds (described and illustrated in section 6 ) in which nestlings rayidly extend and retract their neck several times when greeting each other.

\subsection{Flap Flight Display}

Flap Flight Display in A. ibis is almost identical in appearance to the homologous display in Butorides virescens (Meyerriecks). F.F.D. is a slow display flight of 2 to 10 metres, usually given only in the immediate vicinity of the territory. During the two days immediately before pairing the great majority of flights by both sexes in the colony have F.F.D. components. The following components occur.

1. Deep, exaggerated wing beats which produce loud "thud" sounds, the display's most characteristic feature. The thids are not caused by the wings clapping together.

2. As shown in figure 13 the body axis is at about $45^{\circ}$ to the horizontal and partly extended neck at about $60^{\circ}$.

3. Legs dangle.

4. The beak was open in $40 \%$ of 60 observed ones, but there is no call as in B. virescens. 
FIGURE 13. Flap-Flight Display

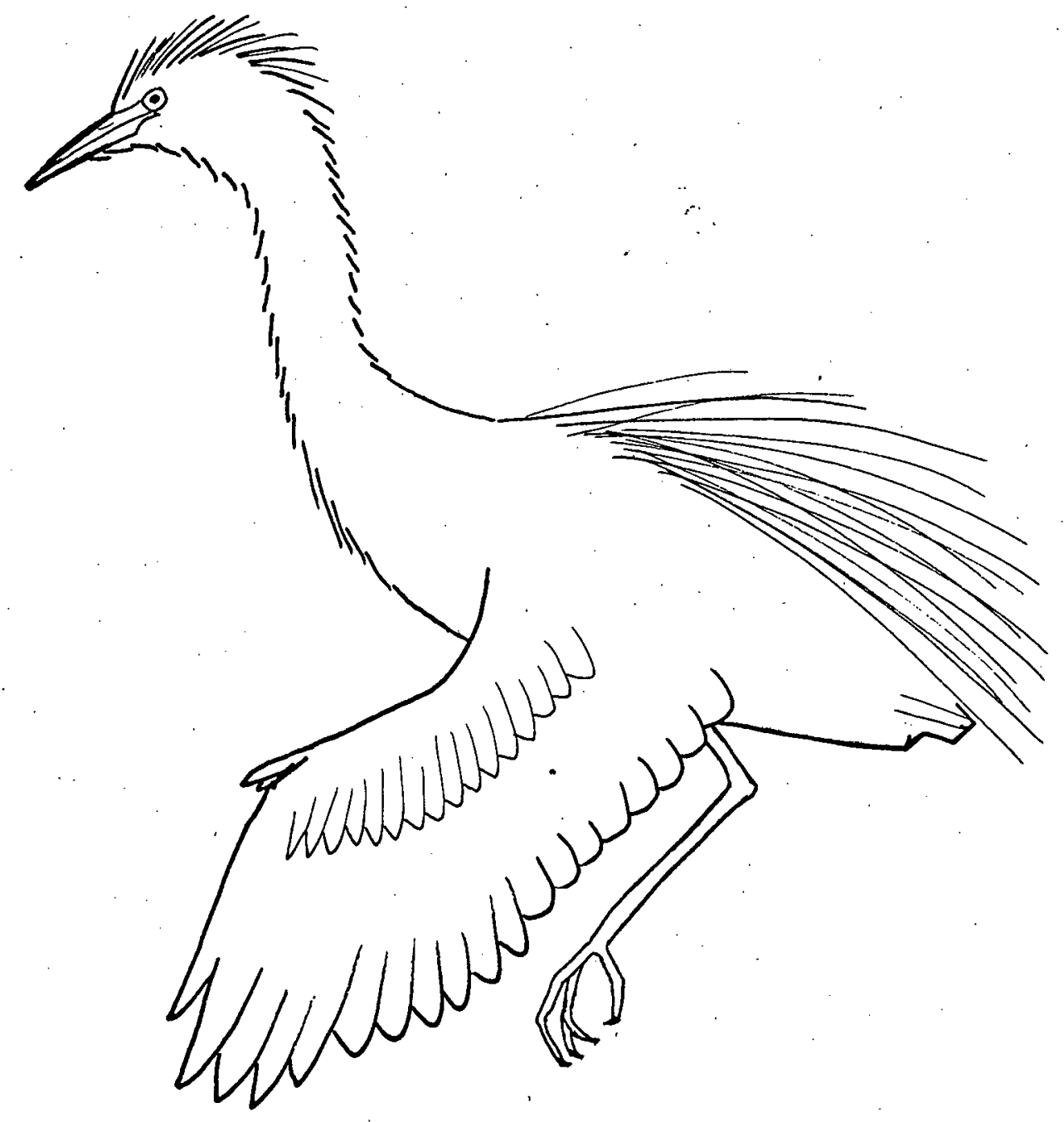


FIGURE 14. Crest positions during Flap Flight Display. 31 displays observed in males.

13 displays observed in females.
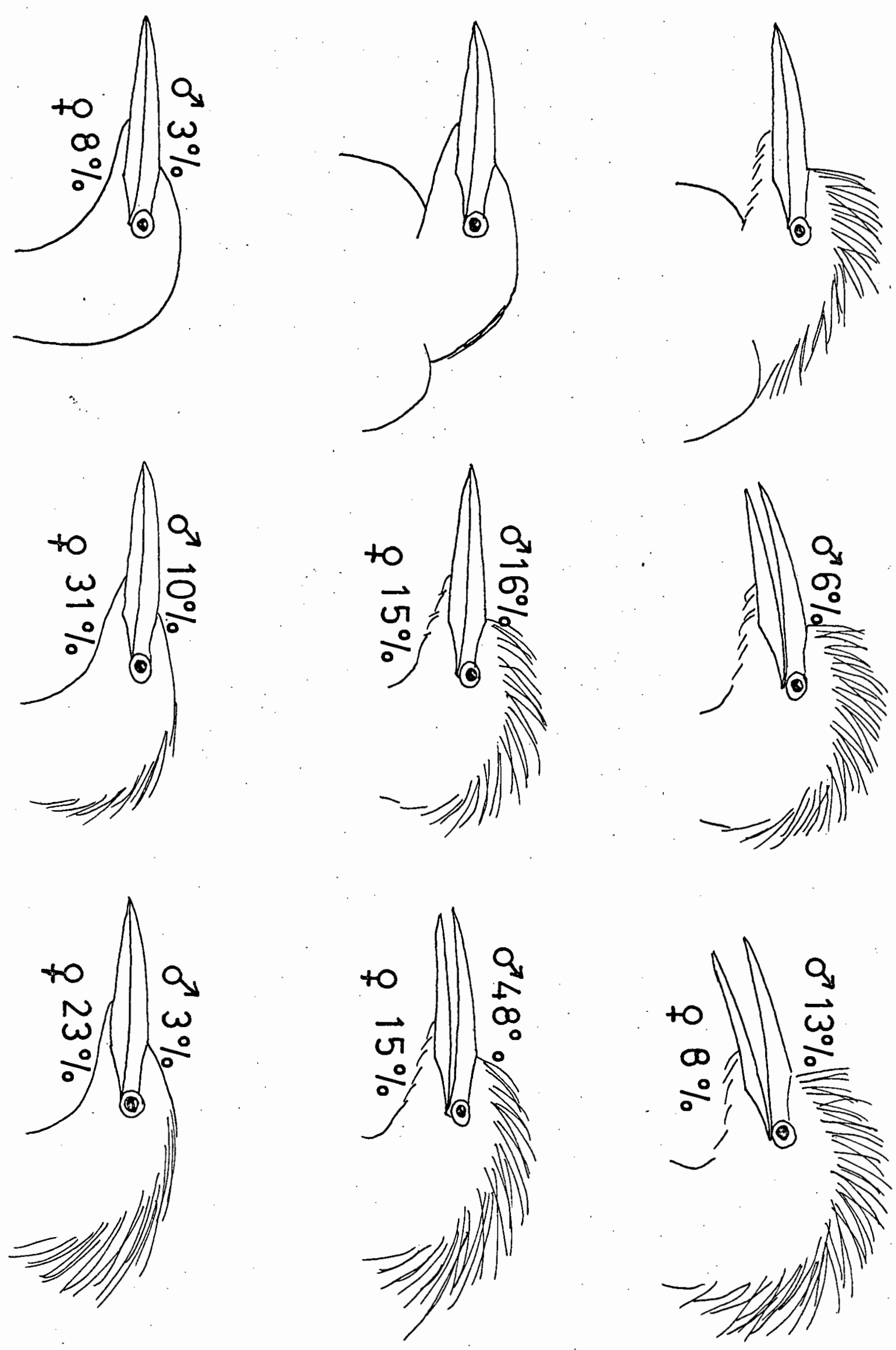
5. The beak is usual].y directed slightly downwarảs, unlike Meyerriecks illustration.

6. Feather positions are variable. Normally the crest (details below), pectoral and scapular plumes are partly raised in flight. All plumes are raised further on the instant of landing and subside gradually over the following 4 to 6 seconds. Crest positions: in flight were recorded in detail in 46 displays and the results are presented in figure 14, which shows that aggressive tendencies predominate in males, where the commonest crest position is 6 and that fear tendencies predominate in females, where the commonest crest position is 8 .

Table 1 shows that F.F.D. is commonest in unmated birds of both sexes. In females the display is inhibited almost completely from the moment of pairing, while males continue to display frequently for a few inore hours. F.F.D. only occurs in red-beaked birds.

Cause. F.F.D. occurs in several contexts, listed in table 3. The majority (63\% of 35) of female displays occur when the bird is approaching an unpaired male, with $47 \%$ of these flights occurring in immediate response to a male's F.F.D. By contrast, only $17 \%$ of 185 F.F.D's in unmated males are directed towards anothex individual ( $14 \%$ supplanting) and are never given in response to F.F.D. in other birds. The fear-aggression motivation of this display emerges from figure 14, discussed above. It is clear from the crest positions that conflict is involved, with aggression stronger in males. In addition, $14 \%$ of male F.F.D's are part of supplanting attacks and the open down-pointing beaks are both aggressive components. Without giving any evidence, Meyerriẹs states that "sexual tendencies prevail". This is unlikely, since there is no trace of the components which suggest sexual tendencies in stretch Display, namely crouching, upwara-pointing beak and flat crest. Site-ownership tendencies are also present since on several occasions: males have been seen to fly towards their nests with normaliy, changing over to F.F.D. for the last few wing-beats before landing.

Function. Because it is both noisy and conspicuous, one would expect F.F.D. to be the chief advertising display in A. ibis. Table 3 shows that a number of approaches by females occur in immediate response to a male's F.F.D. In addition, a female often pays visibly greater attentions to a nearby male when the 
TABLE 3. Situational analysis of Flap Flight Display in Ardeola ibis.

\begin{tabular}{|c|c|c|}
\hline \multirow[t]{3}{*}{$\begin{array}{l}\text { unpaired males } \\
\text { ( } 185 \text { displays) }\end{array}$} & $\begin{array}{l}\text { display not orientated in } \\
\text { relation to any other bird }\end{array}$ & $83 \%$ \\
\hline & $\begin{array}{l}\text { supplants or threatens a } \\
\text { female on landing }\end{array}$ & $14 \%$ \\
\hline & $\begin{array}{l}\text { flying towards a female, but } \\
\text { not supplanting }\end{array}$ & $3 \%$ \\
\hline \multirow[t]{4}{*}{$\begin{array}{l}\text { unpaired females } \\
\text { (35 displays) }\end{array}$} & $\begin{array}{l}\text { not orientated in relation } \\
\text { to any other individual }\end{array}$ & $11 \%$ \\
\hline & flying towards a male & $4.3 \%$ \\
\hline & $\begin{array}{l}\text { ditto, immediately after } \\
\text { a male F.F.D. }\end{array}$ & $20 \%$ \\
\hline & $\begin{array}{l}\text { after having been supplanted } \\
\text { by a male }\end{array}$ & $26 \%$ \\
\hline \multirow[t]{2}{*}{$\begin{array}{l}\text { paired males } \\
\text { (36 displays) }\end{array}$} & $\begin{array}{l}\text { flight away from nest } \\
\text { and mate }\end{array}$ & $50 \%$ \\
\hline & $\begin{array}{l}\text { flight towards nest and } \\
\text { mate }\end{array}$ & $50 \%$ \\
\hline
\end{tabular}


latter performs a F.F.D., changing from a relaxed to a peering attitude, so the display must also have a sexual stimulatory function. F.F.D. cannot have an advertising function in unpaired ferales, which actively search for mates among the relatively stationary males, so the display's function must be purely stimulatory in the case of females.

Origin. The skeletal components of this display differ little from those of unritualised short-distance flights; namely neck partly extended, body not horizontal and feet dangling. In fact, the only positive proof of ritualisation lies in the display's loud wing beats, which are not unavoidable, as A. ibis can fly almost vertically upwards with very little wing-noise. The aisplay can only have originated as short, territory-based flights (which must have been motivated by stronger territorial tendencies than exist in those Ardeid species which have long display. flights).* One functional by-product of these short flights, which may have been supplanting attacks, would have been wing-noises which eventually became exaggerated and ritualised. Aggressive tendencies are strong in unmated A. ibis, so aggressive components (beak and crest) have become part of the ritualised display. Body size has clearly been a factor in the display's evolution. Full intensity F.F.D. has only been recorded in A. ibis (wing length $240 \mathrm{~mm}$.) and Butorides virescens ( $180 \mathrm{~mm}$. ), although weak thudding sounds are sometimes produced by a form of FED in Egretta garzetta $(270 \mathrm{~mm}$.) and E. intermedia (305 mm.).

\section{$\underline{3.5}$ Back-biting.}

Back-biting is a common display in both sexes during the earlier stages of pair relationships. Normally, the displaying bird pushes up against or stands beside its mate and rapidly. runs its partly open beak to-and-fro through its mate's back and/or neck feathers, with sideways head-shaking movements. Quivering open-close beak movements are always present, although the maxilla and mandible never actually clatter together. At higher intensities the mate's neck may actually be grasped and shaken. The display is almost always accompanied by Soft Chatter (section 3.022), which should therefore not perhaps be regarded as a separate display, but as a component of Back-biting. When the level of aggressive motivation is very high, particularly during the first few minutes of pair-formation, the display's 
FIGURE 15. Back-biting: two common positions. (A viewed from above.)
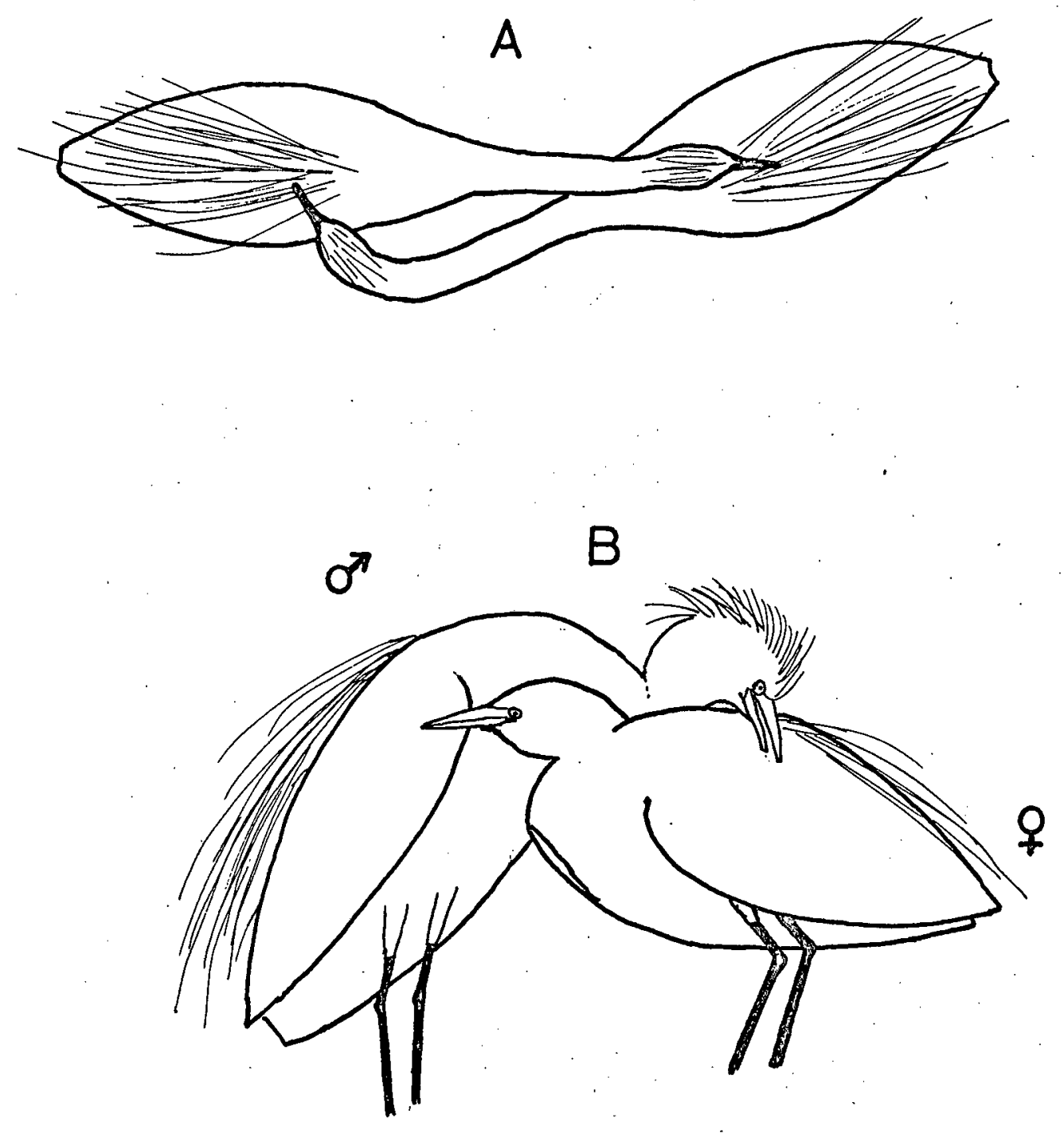
movements are barely distinguishable from overt attack. At low intensities the displaying bird puts its neck across its mate's back or neck and briefly nibbles the feathers on the opposite side. Occasionally these biting movements are performed several centimetres above its mate's back. The usual skeletal attitudes during Back-biting of both members of the pair are illustrated in figure 15, and the commonest crest positions are $2,3,5$ and 6 .

Hudson (1965) and Meyerriecks mention "feather nibbling" in several species and Harrison (1965), partly on this basis, states that allopreening (i.e. mutual preening) occurs in all Ardeidae. These statements refer to Back-biting or homologous displays and since this display is almost certainly derived from inhibitedbiting movements (see below), allopreening probably does not occur in any Ardeidae. A great drop in the frequency of Backbiting occurs after the first few hours of pair-formation; (section 5.32) a drop which coincides with a decline in aggressiveness in both sexes. Throughout pair-formation the display is more frequent in males than in females.

Cause. Table 4 indicates that the majority of Back-bites are either spontaneous or else given in response to the mate's biting. The only situation in which the display is predictable is greeting ceremonies, and during the earlier stages of the pair relationship, greeting ceremonies invariably include Backbiting. The crest positions (usually $2,3,5,6$, ) indicate that aggressive tendencies predominate.

Function. Excluding greeting ceremonies, which in any event frequently contain Back-biting components, this display is the only one which involves physical contact between paired birds, and as such provides strong tactile stimuli, whereas all other displays are visual and/or auditory. The display may therefore have been selected to reinforce the pair bond because it provides a maximum amount of sensory stimuli: tactile, visual and auditory. As regards the display's immediate effects, table 4 shows that females occassionally (19\%), but males never, respond to their mate's Back-bites by crouching submissively.

Origin. Back-biting in A. ibis is clearly related to Bill-clappering displays which have been described for a number of species. In Ixobrychus exilis, both sexes produce a beak "rattling" when greeting, and occassionally "necks may be crossed and the bill occassionally touches the back of the neck". (Mel- 
TABLE 4. Situational analysis of Back-biting.

\begin{tabular}{|c|c|c|}
\hline Situation in which the display occurs. & $\begin{array}{l}\text { male } \\
116 \text { displays }\end{array}$ & $\begin{array}{l}\text { female } \\
75 \text { displays }\end{array}$ \\
\hline $\begin{array}{l}\text { spontaneous } \\
\text { return of mate to nest } \\
\text { Back-biting by mate }\end{array}$ & $\begin{array}{l}71 \% \\
15 \\
15\end{array}$ & $\begin{array}{c}39 \% \\
3 \\
59\end{array}$ \\
\hline Responses to mate's Back-biting & $\begin{array}{l}\text { male } \\
29 \text { displays }\end{array}$ & $\begin{array}{l}\text { female } \\
8 I \text { displays }\end{array}$ \\
\hline $\begin{array}{l}\text { none } \\
\text { also Back-bites mate } \\
\text { crouches submissively }\end{array}$ & $\begin{array}{l}42 \% \\
58 \\
0\end{array}$ & $\begin{array}{l}42 \% \\
40 \\
\quad 19\end{array}$ \\
\hline
\end{tabular}


ler 1961). In Nyctanassa violacea, "the herons stood side by side and exchanged stroking gestures in which each passed the side of the beak downwards against the primaries of the other's folded wing" (Harford 1951). In Ardea melonocephala "head and neck wound over the back of mate and bill ran up and down back and opposite flank of mate with clashing of bill" (Symmes 1951). In Florida caemlea "Both birds crossed their outstretched necks and kept up a continuous rattling chatter for several minutes, biting at each other's plumes at the posterior part of the body" (Meanley 1955). In Egretta, the displaying bird usually performs rapid rattling mandible movements while standing next to its mate, but feather-touching seldom occurs (this study). The most detailed description is for Ardea cinerea (Hudson 1965). The above displays can be arranged in a series: back-feather biting (A. ibis); feather-biting with rattling beak sounds and usually with necks crossed (most other species); beak-rattling sounds alone (Egretta). In addition there is a form of Backbiting in A. ibis in which the displaying bird does not actually toush its mate but performs biting movements a few centimetres above its back. All the above strongly suggests that Ardeola Back-biting and Egretta Bill-clappering are homologous, and that there is a full range of intermediates in other species. In adition, all the displays characteristically occur in greeting ceremonies.

Hudson (1965) concludes that Bill-clappering in Ardea cinerea is derived from allopreening, but the above evidence suggests that one form of the display series, i.e. Back-biting in Ardeola ibis, is derived from bitins movements.

(a) the beak is opened more widely than in preening, and the movements are far more similar to biting than to nibbling preening. At high intensities, the beak actually encloses the mate's neck.

(b) the sideways head movements, also noticed in Ardea cinerea, are characteristic of inhibited biting.

(c) Back-biting is commonest duxing the start of pair-formation in A. ibis, when agoressive tendencies are highest.

All the above suggests that Back-biting in A. ibis is derived from inhibited biting movements, although one cannot entirely reject the possibility that the display was derived from allopreening which has been modified by aggressive tendencies. Since the two displays are homologous, Bill-clappering 
must also be derived from inhibited biting.

\subsection{Twig Shake}

The perched bird stretches out its neck almost to full extension, grasps a leaf or twig in its beak and shakes it with sideways head movements for 1 to 3 seconds. The grasping may be directed upwards (49\% of 199 observations), in front of the bird $(43 \%)$ or downwards $(8 \%)$. Figure 16 illustrates these three positions, the divisions between which are necessarily arbitrary. Back-and-forth jerking movements of the head are also present while the twig is being shaken and in a few cases (estimated 10\%), may predominate over the sideways components. Scapulars and pectoral plumes are partly erected; while the crest may be in positions 2, 3, 5 or 6 (fieure 3 ). In $31 \%$ of 148 observations, the display was accompanied by Nasal Chatter (section 4.5); in the remainder, the bird was silent. In unmated males, swaying movements often occur after Twig Shake (section 5.31). The display is distinct from nest-biting movements (described in section 3.01) given during greeting ceremonies.

Table 1, which gives the display frequencies yer hour for both sexes, both when unmated and during the pair-formation, shows that the display is one of the commonest.in unpaired males, and that there is a $99 \%$ fall in display frequency after the males have paired.

Cause. It is clear from the crest positions (2, 3, 5, and 6 in figure 3 ) that aggressive tendencies predominate in this display. In addition, Twie Shake, performed by a male immediately after it has threatened a female (details in section 5.3)' are noticeably more violent than average. Nest-building tendencies also exist, since at lower intensities Twid Shake is indistinguishable f'rom trembling and push-pull nest-building movements, described in section 5.5).

Function. It is unlikely that the display has a longrange advertising function, for the actual movements involved are inconspicuous, and except when accompanied by Nasal Chatter, it is silent. Twig shake by an unpaired male probably has a stimulatory effect on a female which is already attending, (details in section 5.31). The display has no nest-building effect, although leaves are occassionally pulled off in the course of the display. 
FIGURE 16. Twig Shake, giving the relative frequencies of three positions.
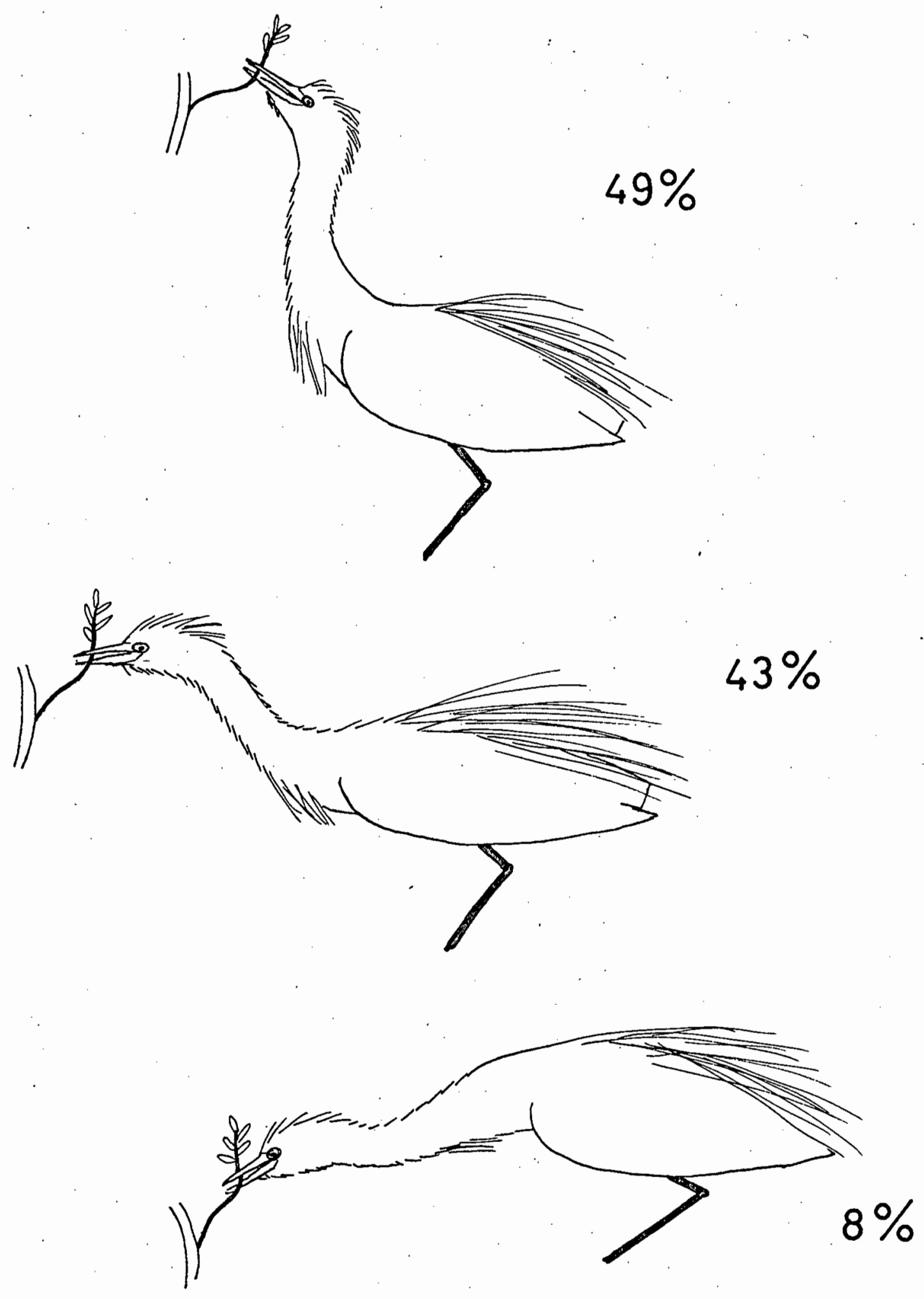
Origin. The "down" position of Twig Shake is similar to the Snap Displays of Butorides virescens and Ardea herodias (Meyerriecks) and A. cinerea (Baerends anả van der Cingel 1962), but there are 5 points of aifference.

(a) Snap Displays (Meyerrieck, Baerends and van der Cingel. 1962, Verwey 1930 and pers obs.) invariably have a form similär to the "down" position in figure 16, while only $8 \%$ of Twig Shake are directed downwards, (b) Snap Displays have a legflexing component (the "bob") which is absent in Twig Shake, (c) Snap Displays are not accompanied by any call, (d) twiggrasping is not usual in Snap, but invariable in Twig shake, (e) Egretta intermedia, which performs typical Snap Displays, also performs Twig Shake, yet not intermediates between the two types of performance have been noticed. The displays therefore differ sufficiently to warrant different names.

In a detailed analysis of the phylogenetic origin of Snay Display, Baerends and van der Cingel (1962) conclude that it is derived from a combination of ambivalent movements and redirected attack (Bastock, Morris and Moyniham 1953). Redirected attack components must be present in Twig. Shake a.lso, for when the aggressive tendency is strongest (see under "Cause" above), the movements resemble biting movements.

Nest building components are also present in Twig Shake, and it is possible that they became incorporated into Twig Shake via a process similar to the one found by Tinbergen (i95.9. 1960) in Iarus argentatus. In this species, redirected attack takes the form of grass pulling. When it has torn out a tuft of grass, the gull usually terminates the action with a sideways flick of the head, very similar to a nest-building movement. Tinbergen's interpretation of this is that the sideways flick is stimulated by the "hest material" that the bird finds in its mouth following an attack on the ground. Grass pulline is therefore described as redirected attack followed by displaced nest-builaing movements. The situation in A. ibis is probably analagous.

Twig Shake almost certainly originated as redirected attack, very similar in motivation and appearance to snap Display. Nest-building tendencies bacame super-imposed on the aggressive tendencies, via a process similar to that suggested by Tinbergen (op. cit.), and the movements became ritualised. Finally the presence of nest-building tendencies caused the 
display's grasping movements to be directed at the nearest twig or leaf, instead of invariably downwards, as in Snap Display.

\subsection{Head Flick.}

This display consists of rapid, small-amplitude head movements in a horizontal plane, given with the beak closed, and may be performed singly or as rapidly as twice per second. It is superimposed most commonly on $5,6,8$ and 9 (figure 3 ), and is commonly associated with low-intensity Withdrawn Crouch. The display occurs in adults in the following. wide range of contexts listed in the approximate order of Head Flick intensity, although it is of course not invariable in any of these contexts.

1. Predator near nest.

2. Immediately following threat by another bird.

3. Immediately after threatening but failing to dislodge a trespasser.

4. In male after attempting to mount an unreceptive female.

5. In unreceptive male, following soliciting by female.

6. Trespassing in absence of nest-owner.

7. Watching, as opposed to participating in, a fight.

Cause. It is obvious that the display is associated with strong conflict situations. In the display's commonest contexts (1, 2 and 3$)$, at least two tendencies are present: flee from the unpleasant stimulus, and remain on the nest. Aggressive tendencies are clearly present in contexts 1 and 3 , for both predators and conspecific trespassers are threatened, so a fight - flee - stay conflict exists. Situation 4 represents thwarted sexual drive, situation 5 a stimulus stronger than the bird!'s tendency to act.

The exact motivation of the display is clear from observations on Head Flick in young birds. Alnestling younger than about 10 days will sometimes threaten and peck at a hand or any other large foreign object held immediately obove the nest. If the object is moved closer and the nestling struck lightly a few times, the threats become weaker, head-flicks more frequent and the nestling usually crouches submissively. A similar effect was seen many times in tame fledglings 20 to 50 days old. When a predator. (domestic cat or crude predator-model) was 
presented to a bird it would give high-intensity threats and peck at the predator. When the latter was moved closer, threats became weaker and less frequent, many Head-flicks would be given and the bird eventually retreated or crouched submissively with Head-flicks. It is clear that when the aggressive tendency began to wane rapidly, and the fear-tendency to increase, Head-flicks was elicited. In concise terms, Headflick is usually produced by intimidation.

Function. In its common contexts ( 2 and 3 ) of agonistic encounters between neighbours, performance of Head-flick by one or both birds marks the end of the aggressive outburst. A trespasser which gives the display only does so while retreating. Both these points suggest that the signal function of Head-flick is "I am unwilling to fight", (Antropomorphisin is not implied, cf. Tinbergen 1959, 25). The occurrence of the display in non-agonistic situations (copulation, 4, 5, nest-building, 8), suggests a wider signal value of "I am unwilling to continue. this activity". As a displacement activity (below), the display presumably has the effect of relieving conflict.

Origin. The components of Head-flick are similar to the movements with which A. ibis will "spit out" noxious animals (e.g. miliipedes) and also discard dried faeces from the nest. There is, however, little rotivational similarity between these two activities and Head-flick, so the most probable conclusion is that the display originated as irrelevant (displacement). spitting-out.

\subsection{Wing-Touch}

Wing-touch is a very common activity in both sexes for the two days preceding pairing. Possibly because the activity is not ritualised and could easily be overlooked as an incidental preening movement, it is not mentioned by Meyerriecks. The following components occur.

1. The bird turns its head to one side and mans its slightly opened beak downwards along the leading edge of the folded wing. (Figure 17). Less often, the secondaries or wingcoverts are toluched. In 223 observations, 118 movements were made to the right and 105 to the left (difference not significant)

2. The carpal joint is always held slightly away from the body while the wing is being touched. 
FIGURE 17. Wing-touch

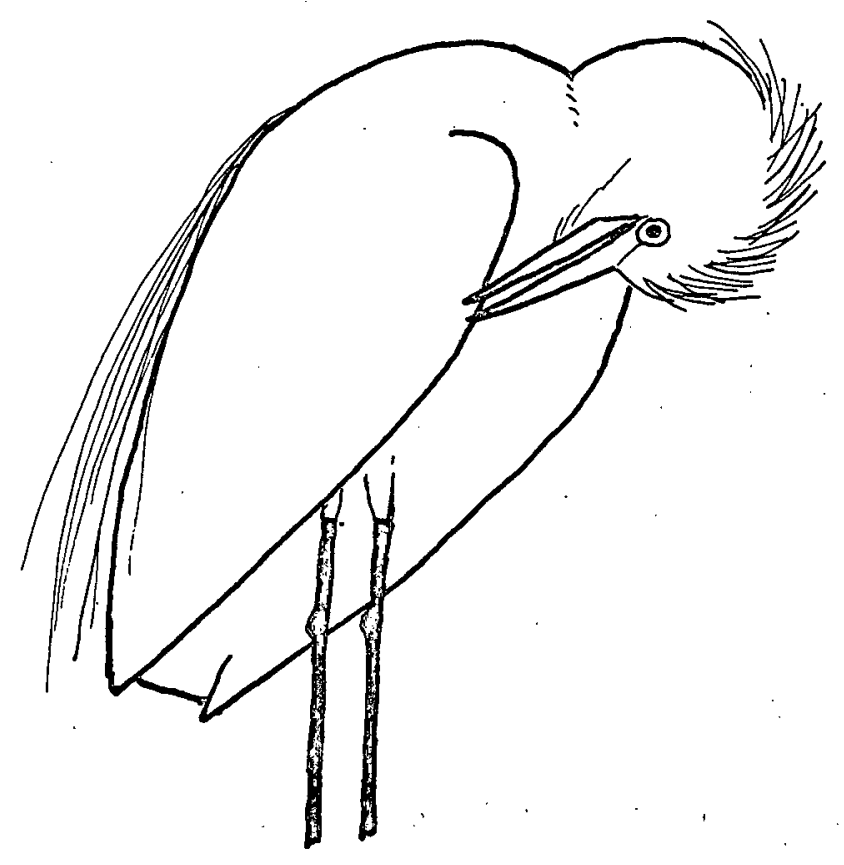

FIGURE 18. Wing Spread

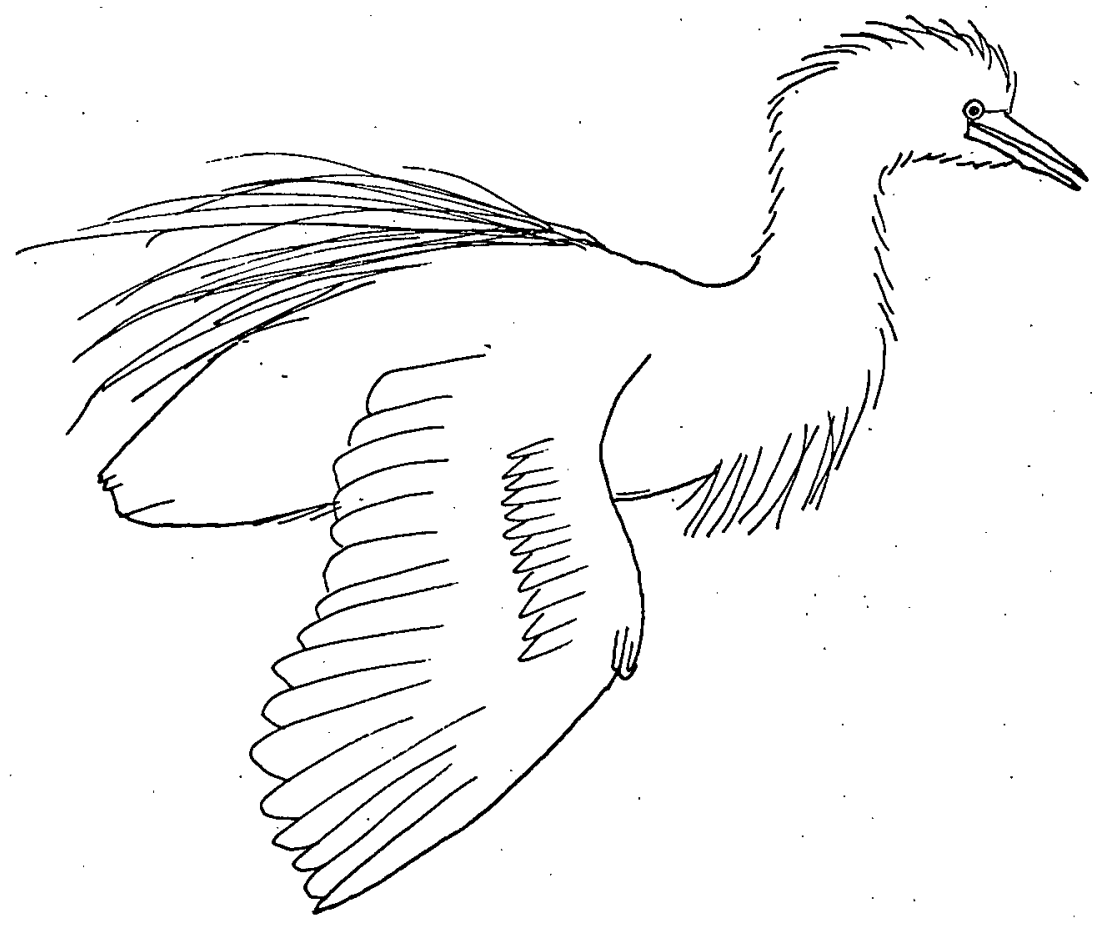


3. Crest position 2 (Figure 3) occurred in $60 \%$, position 3 in $24 \%$ and position 5 in $16 \%$ of 25 wing-touches.

Table 1 shows that the display is very cormon in unmated males and fernales, becoming relatively rare as soon as the birds are mated. Wing-touch only occrs during the red-beaked phase, but an identical movement has been seen several times in a three-month old tame bird in mild conflict situations.

Cause. Table 1 shows that in males the display is strongly stimulated by the presence of a female. In males, only $21 \%$ of 210 Wing-touches were directly associated with another activity, either by the performer or any other individual, so that the display usually appeared spontaneous. The commonest context in males was immediately following threat or supplanting of a female, and in females the corresponding figure was $25 \%$ ( 79 displays), the commonest context being imnediately after supplanting by a nale. Crest positions show the display to be motivated by moderate aggressive tendencies, with little fear involoved. In males; site-attraction tendencies must also exist, otherwise the bird.would repeatediy be making supplanting attacks on birds nearby. In unpaired females, which do not own territories, the conflict is probably between aggression, fear and the sexual attractiveness of the male.

Function. Wing-touch never has any visible effect on any other individual. While the display must obviously have some function, it cannot be very important, which would account for its insignificant movements and low degree of ritualisation compared with most displays.

Origin. Wing-touch is obviously a preening movement, since no other activity involves contact between beak and wing. The display contains all the characteristics of a displacement activity, namely an irrelevant movement occurring when two or more conflicting tendencies are activated.

\subsection{Wing spread.}

Wing Spread is not obviously ritualised but has a definite signal value, being one of the most characteristic behaviour patterns of unpaired males. It also occurs less frequently in paired birds of both sexes during the red-beaked phase, and very occasionally in yellow-beaked birds (section 5.1). The display, illustrated in Figure 18, characteristically contains 
the following components.

1. Wings almost fully spread for 1 to 5 seconds, usually: with rocking movements exactly as if balancing the walking bird.

2. All plumes partiy to fully erect, crest position corresponding to 2 and 3 (Figure 3 ).

3. Neck never more than half extended, beak always pointing downward, the bird standing or walking in the peculiar "hunched" attitude characteristic of unmated males (details in section 5.31 ).

Cause. The commonest situations are: immediately following a Flap Flight Display; while walking towards another individual prior to threatening it; while walking down to the nest immediately after landing in the vicinity. Aggressive tendencies are strong, as indicated by the down-pointing beak, crest positions, and possibling also the wings-spread component itself.

Function. Wing spread almost certainly still serves its original function as a balancing action, since it only occurs while the bird is moving, but it has become one of the most characteristic activities of unpaired males, so presumably has an advertising function.

Origin. Wing Suread is little removed from the normal wing-balancing movements often given after landing, for immediately after alighting or while walking along a shaky perch; a bird will momentarily extend one or both wings to maintain its balance. These movements have undergone little exaggeration, but have instead become very common in some situations as a result. of lowered threshold, one of the four processes which Daanje (1951) considers are involved in display ritualisation.

\subsection{Greeting Ceremony}

There is always ritualised displaying when a paired bird returns to its mate on the nest. No single activity is involved; instead, components from a number of displays usually occur in rapid alternations. During brief absences, usually when the male has fetched nesting material, the displaying consists only of a few Rick-racks by both sexes when the incoming bird walks onto the nest. After long intervals of separation displaying is more prolonged and intense. Normally the incoming bira begins to Rick-rack while still 2-10 metres away and 
FIGURE 19. Characteristic positions during a greeting ceremony in A. ibis.

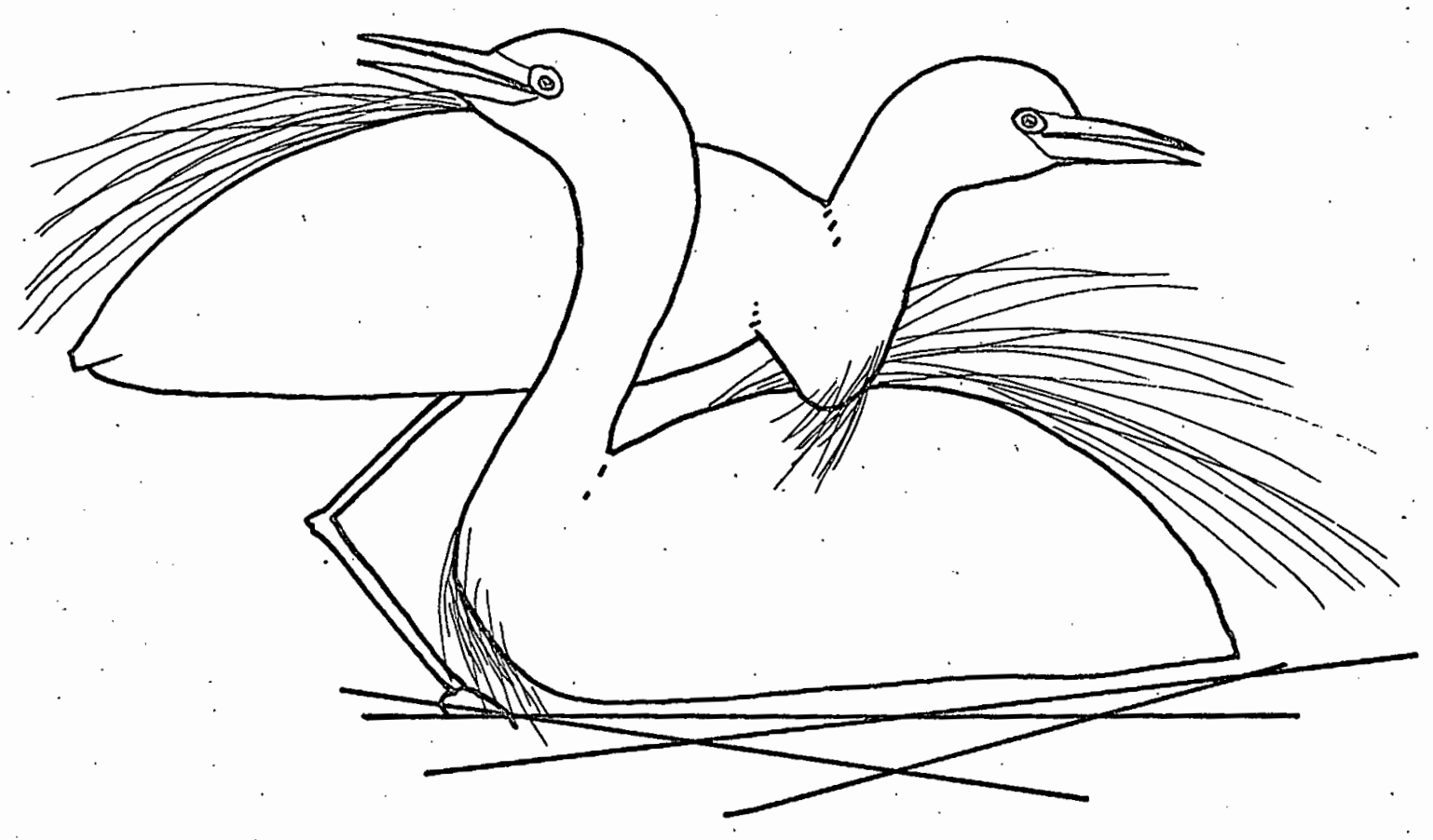


flying towards the nest. The sitting bird looks up, begins to Rick-rack as its mate walks towards the nest, points its head and neck upwards in a low intensity stretch movement then squats downwards, simultaneously giving the harsh "roo" call. "Roo" calls were given in $73 \%$ of 85 greeting ceremonies, and in $11 \%$ of the 85 cases the incoming bird also performed a low intensity Stretch Display with call. The incoming bird then stands, with flexed legs, beside its mate, usually with their necks partly crossed as in figure 19. Particularly during the earlier stages of the pair relationship, mutual Back-biting occurs. (Backbiting has only once been observed in a pair with younf). After a few seconds of this behaviour, loud Rick-rack is replaced by Harsh Chatter ("kakakak") in both birds. The crests of both birds are always completely flat throughout greeting, and their scapular plumes partly to fully raised.

At some stage during the greeting ceremony the sitting bird usually grasps a part of the nest framework in its beak and shakes it briefly with "tremble-shove" nest-building movements (section 5.5), quite different from the movements of Twig Shake. This presumably represents unritualised displacement nest-building. During the first few days of the pair relationship, when both bird's beaks are red, all calls are muffled and husky; (Soft Chatter, quiet "roo" and hoarse Rick-rack), Back-biting predominates, and Stretch is given infrequently.

After the calling and displaying has abated, the departing bird slowly walks away from the nest and usually perches in the treetop for a few minutes before flying off. The incoming bird usually tremble-shoves the nest material for a while and may turn the eggs before sitting. Greeting ceremonies always follow the same pattern regardless of the sex of the incoming bird.

In 5 greeting ceremonies timed during incubation the calling lasted an average of 15.3 seconds, 7 timed for pairs with chicks 1-10 days old lasted an average of 14.5 seconds, and 8 timed for pairs with chicks 11-20 days old 18.5 seconds. The differences are not significant. As in Ardea cinerea (Verwey 1930), adult Ardeola ibis do not exchange greeting calls once the young have left the nest and it may be assumed that the pair-bond is then dissolved.

Greeting ceremonies have been described in several Ardeidae; in greatest detail for Ardea cinerea (Verwey 1930: a major 
paper which the present author was unfortunately unable to consult in detail, as it is in German). In Greeting in colonial-nesting A. melanocephala (North 1963), the incoming bird gives loud "kow-owk" alighting calls, and the bird on the nest gives a brief silent Stretch display. In Florida caerulea (colonial), "elaborate greetings were exchanged. The pair would repeatedly call "quip-a-quee" to each other, peck at one another's plumes and cross outstretched necks" (Meanley 1955). Meyerriecks mentions greeting ceremonies in Butorides virescens (semi-social, cxyptic), only stating "Stretch Displays appear during nest relief". In Ixobrychus exilis (solitary nester, highly cryptic) "The bird on the nest calls a low gra-a-a". Slisht rattling bill-noises are made (Weller 1961). Observations on the above four species, Ardeola ibis and Esretta garzetta (this study) indicates that greeting is noisy and conspicuous in colonial Ardeidee, and very quiet in solitary, cryptic species. The "noisiness" of greeting in colonial species probably reflects removal of the selective effects of predation.

The fully flattened crest which is characteristic of greeting in A. ibis is significant in two respects. Firstly, it provides a good illustration of the principle of "reversed movements" which was developed in section 3.2. Crest-erection, particularly of the anterior region, indicates aggressive tendencies so complete inhibition of crest erection presumably indicates non-aggression or appeasement. Secondly, in all other Ardeidae in which the point has been recorded, the crest of both sexes are partly to fully erected during greeting: Ardea melanocenhala (North 1963), Ixobrvchus exilis (Weller 1961), Egretta intermedia (no crest, but crown feathers raised) and E. garzetta (both in this study). Information is at present too scanty for conclusions to be drawn, but the above may indicate that Ardeola is quite distinct from the rest of the tribe Ardeini: (Bock 1956). Greeting ceremonies and all other displays which serve to maintain the pair bond are held to be particularly useful indicators of phylogenetic relationships (Johnsgard 1965).

Scapular plume erection occurs in most displays: Forwara, Stretch, wing-touch and Wing Spread, and in the greeting ceremonies. It was originally assumed that there plumes have some stimulatory effect, but their removal in incubating birds (one of each sex, different pairs) did not have the slightest observable effect on the mate's behaviour. 
3.021 Rick-rack

A. ibis' commonest call has been named Rick-rack by Skead (1966). Normally, it is a harsh double croak with the first syllable louder and higher-pitched than the second, but during the red-beaked phase the call is quieter, muffled and hoarse, giving a low-pitched "Ruk-rok" sound, (presumably the "Kungkung" call Valentine 1958 mentions). This variant of Rick-rack is extremely common in the earlier stages of a colony's development. Rick-rack is given throughout the year at roosts and nesting colonies, but is seldom heard among feeding birds. The call j.s variable and overlaps with "Kok".

Cause. The call occurs in too many contexts to make a list meaningful, but it is always a component of greeting ceremonies (details in section 3:01). Rick-rack is invariably given by a bira returning to its nest, even after an absence of as little as 10 seconds, regardless of whether its mate is present or not, so site-ownership tendencies must be involved. Iittle else can be said regarding notivation, as the display is not associated with any particular behaviour pattern, but the call is absent in birds with strong fear tendencies.

Function. One definite function of the display lies in individual recognition, for a sitting bird which has not noticed its mate arrive will look up when the mate calls and Rick-rack in reply.

Very occasionally, short croaks are given by individuals flying away from the colony, and less often by gatherings of A. ibjs on their feeding grounds, when the calls may be associated with the arrival of a bird. These croaking calls appear to be a low-intensity form of Rick-rack.

\subsection{Chatter}

Three tonally similar calls, none of them previously described, are grouped under this heading. Nasal Chatter, which occurs only in unmated males, consists of a 1-2 second phrase of 5-12 flat, nasal notes, descending in volume and frequency In Soft Chatter, restricted to recently-mated red-beaked biràs of both sexes, the call is quieter and does not descend. Harsh Chatter, occurring in yellow-beaked mated individuals, differs from soft chatter in beinf loud, harsh, slower and longer 
approximately "kakakakakak".

Table 1, which gives the frequencies of Nasal and Soft Chatters, shows that the presence of a female stimulates Nasal Chatter in unmated males. Harsh Chatter occurs only in greeting ceremonies.

Cause. Table 5 lists the contexts in which these two calls occur: $42 \%$ of Nasal Chatters are associated with iwig Shake and $31 \%$ with landing on or walking back to the territory. These figures only inform one of the general situations in which the display occurs and are not intended to imply that the call is actually caused by landing on the territory. As in most activities occurring in males during the rea-beaked phase, afgressive tendencies predominate, as shown by the crest which is usually in positions 2 or 3 (Figure 3 ). The great majority (87\%) of Soft Chatters occur simultaneously with Back-biting.

Function. Nasal chatter is a very characteristic call of active unmated males and can be heard at distances of 40 metres; so is vresumably an important advertising display. The switch from Nasal to Soft Chatter following acceptance of a female must be correlated with a change in function, probably from advertising to sexual stimulation.

\section{$3.023 \mathrm{Kok}$}

A. ibis has two alarm calls, the conmonest of which is a low-pitched "kok" note, given singly to as rapidly as three per second for minutes at a time. Sound production is accompanied by a slight bulging at either side of the neck.

Calase. Kok is always associated with crest and neck positions 4 or 7 , indicating a moderate to high fear tendency. The call is commonest at the nesting colony but also occurs at stick-collecting sites (section 5.5) and roosts, and is readily elicited by the presence of a predator or human intruder near the nest.

Function. By means of the call, information on the presence of a predator is rapidly spread throughout a colony, for if one bird begins a rapid series of koks, all individuals in the vicinity begin to call and walk away from their nests.

Origin. Very similar alarm calls occur in Ardea cinerea (Witherby et al 1940), A. herodias (Cottrjlle 1958) and A. melanocephala (North 1963). In the case of many passerines, 
interspecific similarities between alarm notes are due to the fact that they tend to make the location of the caller difficult (Narler 1956a), so that similarities are presumably due to convergence. Iocation of Ardeidae, particularly white ones, is an easy task for any predator that hunts by sight, so it is highly unlikely that the nature of the alarm call could affect the vinerability of the caller. In this case the alarm calls of the four species are probably homologous and since Ardea and Ardeola are not closely. related the call must be a primitive one.

\subsection{Ka.eah}

A much rarer alarm call than Kok is a harsh, drawn-out "Kazah" note, which differs from the normal call of Forward Display only in being much louder and longer. The associated motor patterns at full intensity are: flattened anterior crest, partly raised posterior crest, fully erected neck feathers and rarely, stabbing beak movements and weak wing beats, similar to those in Forward Display. The neck may be either retracted or: extended.

Cause. Kaaah is usually elicited by the presence of a predator such as a bird of prey or domestic cat (experimental) very near the nest. This call is motivated by conflict between fear and much stronger aggressive, parental and/or site attachment tendencies than occur in Kok. High-intensity fear-aggression conflict is evident from the crest position ( 8 and 9 in figure 3) and the wing-waving component also indicates a degree of aggressiveness absent in Kok. Parental tendencies also play a part, since the tendency to give this display becomes emphatical.ly greater shortly after the eggs hatch. A similar effect is seen in figure 8 which shows a slight increase in the frequency of Forward Displays at this stage.

Function. The display's intra-specific warning effect is similar to that of Kok, only more marked. Kaaah also has an inter-specific signal function, referred to in section 2.4,and in a field test with a domestic cat the noise (mostly Kaaah) of nearby nest-owners clearly had a distracting effect on the cat, reaucing its tendency to explore.

Origin. The cail and its associated components are clearly derived from Forward Display which has been modified by fear and possibly also by parental tendencies. The call itself,'crest positions, and in some cases head movements and partly spread 
wings, all have easily recogniseable counterparts in Forward Display. The high intensity "Kaaah" alarm call which North (ig63) mentions in Ardea melanocephala is probably homologous. 


\subsection{Daily routines and roosting behaviour}

The flock movements and daily activity cycles of A. ibis were not studied in detail, as these aspects were covered by another long-term study in the same area at the same time.

Throughout the year A. ibis is gregarious, sleeping in roosts which vary in size from about 20 to over 5,000 birds. Niost roosts known to the author are in trees or reedbeds surrounded by or in immediate proximity. to water. Every morning the roosting birds disperse to their feeding areas, leaving the colony singly or in small straggling groups which later split up. The greatest distance feeding A. ibis have been observed from the nearest roost is $22 \mathrm{~km}$., which roughly agrees with the distances of "about 12 miles" given by Skead (1966) and Crauford (1966).

There is very little behavioural interaction among feeding birds although Supplanting Runs (section 3.22) and flightchasing occur when two individuals compete for a single food item. Flock movements are not highly synchronised and there are no flight calls. Individuals move to different feeding areas many times in the course of a day, so that the size of flocks varies greatly from one hour to the next. When a member of a gregarious species becomes separated from its flock, it shows signs of restlessness. Solitary Parus major, for instance, hop restlessly, give flocking calls, and peer around (Hinde 1952), but solitary A. ibis show no such behaviour.

The average size of flights can be used as an index of the degree of gregariousness of a bird species, for there is no possibility that the birds are simply aggregating, which is often the case in feeding birds. Figures were therefore obtained on the size of flocks (August 1966 to March 1967), observations being restricted to the periods 0900 to 1600 hours to exclude birds moving to and from roosts. During the breeding season, birds flying directiy to or from nesting colonies were also excluded. Single flying birds were observed on 64 occasions, two-bird flights on 22, three-bird on 15, and five-bird on 4 occasions. Once ( 1125 hours on 16.9.66) a feeding flock of 31 birds flew up simultaneously (no disturbance was detected by the observer) and circled once before flying off. This was the largest flock of birds that has been observed, excluding 
evening roost-bound flights, and the only occasion in which a synchronised flight has been observed among feeding birds.

The weak flocking tendency in flying A. ibis shows that the species is only slightly gregarious when not roosting or nesting.

Between 1100 and 1400 hours A. ibis sometimes gather and rest in flocks of up to 200 birds, but this is not invariable. These gatherings usually develop in feeding areas or on the banks of dams, and there is no interaction other than occasional supplanting runs and Rick-racks from arriving birds. As Vincent (1947) points out, these midday rest periods are to a certain extent a reflection of the inactivity of cattle at that time. The present author obtained the strong impression that the birds feed over a wider area and for longer hours in the ary months December - Narch.

About one hour before sunset the birds begin to fly towards the roost; the tendency to form flocks is far stronger in birds flying towards roosts or nesting colonies than in those flying away. Crauford (1966) found that all birds fed within 35 mins. flying time of a roost in Sierra Leone. Birds seldom fly direct from their feeding areas to the roost but usually congregate at gathering points one to ten kilometres from the roost, up to 300 birds accumulating before the whole lot straggle onwards, coalescing with other flocks en route. Gathering-points may be in tall trees or open grassland, and are to a large extent traditional but the birds often alternate gathering points from one day to the next. Vincent (1947) and skead (1966) also observed this system of traditional roosts and pre-roosting congregations whose positions change. A similar system exists in some Corvidae (Burns 1957). No function for the habit is suggested.

Crauford (1966) states that roost-bound flights of A. ibis tend to follow water-courses. The present author is not convinced of this but it is clear that A. ibis avoid flying over high ground. In the Paarl area the birds never flew over hills more than about 100 metres above the surrounding plain, even though the detour entailed a flight of up to $8 \mathrm{~km}$. extra for some biras. Unless strong winds interfere, the great majority of roost-bound birds fly between 10 and 100 metres altitude.

Most birds arriving at the roost fly direct to a perch but some pause nearby to arink. There is little aggression among roosting birds; if one individual lands near another's perch it 


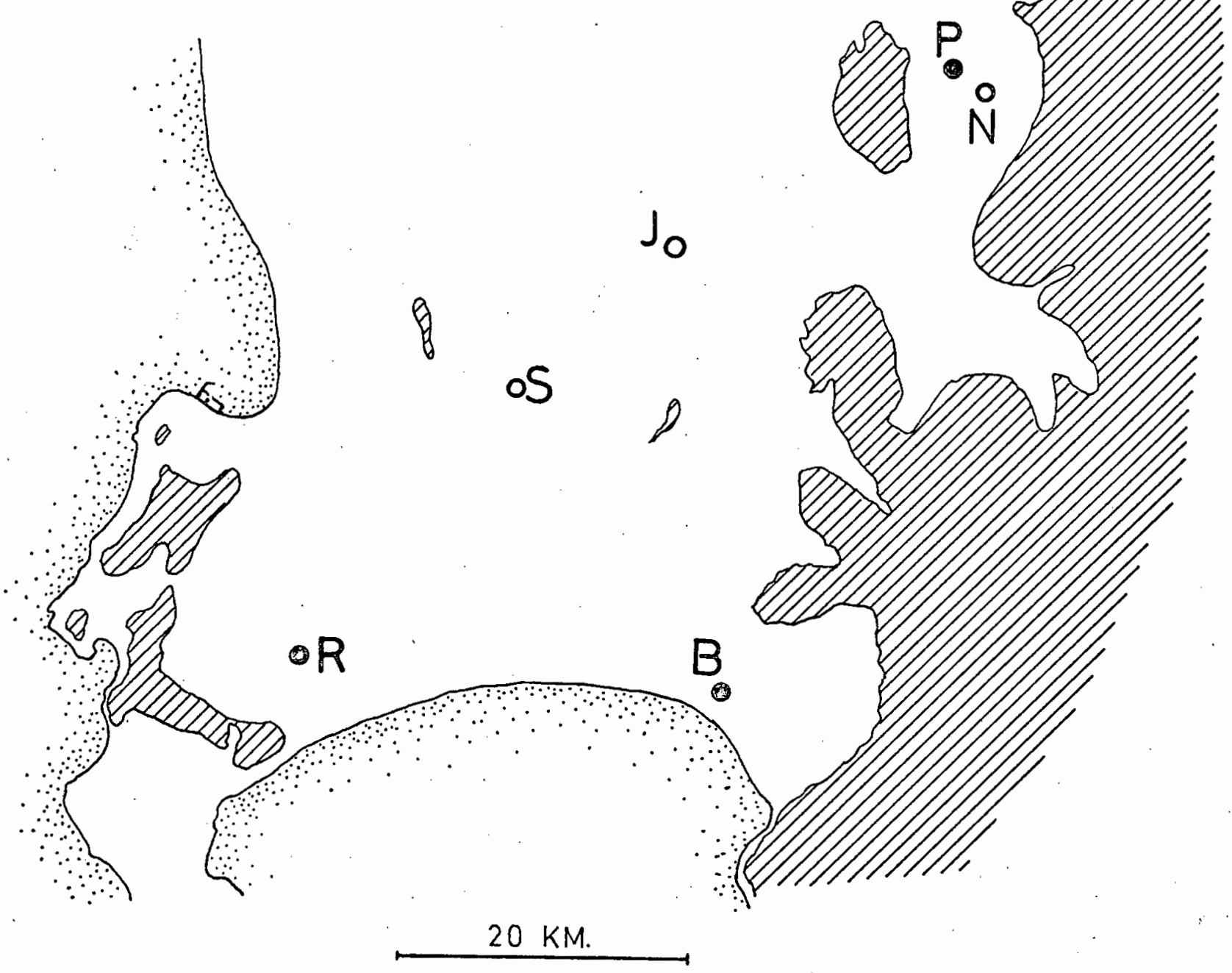

FIGURE 20. Map of the study area, indicating the six A. ibis roosts at de Beer's (B), Joostenberg ( $J$ ), Nederburg $(N)$, Paarl (P), Rondeviei $(R)$ and Stikland $(S)$. Roosts also used as colonies indicated by solid circles. Hatched areas: regions over $330 \mathrm{~m}$. altitude. 
is supplanted but fighting is rare. Arrival of each group of birds in the roosting trees is accompanied by many "raa" calls of Forward Displays by birds defending perches but the disturbance soon dies down. Rick-rack is common among roosting birds and there is a contimuous background of low "gobbling" notes which could not be identified with certainty, but are probably subdued Rick-racks. There is little calling and movement at night, but once (at full moon) the author observed a group of about 30 birds flying away from a roost.

Figure 20 gives a map of the study area (approx. 2000 square km.) and names the six roosts in existence in August 1966: at de Beers, Joostenberg, Nederburg, Paarl, Rondevlei and Stikland. The feeding ranges of birds from adjacent roosts are to a large extent mutually exclusive, but birds can frequently be observed crossing "boundaries" at any time of day or year, so some inter-roost movenents probably occur, as in Corvus frugilegus (Burns 1957).

During the breeding seasons there are nesting colonies at the de Beers, Paarl and Rondevlei sites and in each case the initial nucleus of the nests is 2 - 400 metres from the roosting trees. From August onwards the numbers of birds at Joostenberg, Nederburg and Stikland begin to decline steadily as the birds moved to the nesting areas, but it is not known for certain whether these roosts are ever vacated entirely at any stage, although only $15 \mathrm{~A}$. ibis were present at the Nederburg roost on 17.10.66. From mid-November onwards the numbers at Joostenbery; Nederburg and Stikland roosts increase with the arrival of young birds and the return of adults which have completed breeding. With the complete desertion of some roosts during the breeding season, the feeding ranges covered by the birds roosting and breeding at de Beers, Paarl and Rondevlei increases.

\subsection{Flock sizes and host relationships}

A. ibis is best known for its habit of associating with large herbivores, which has led to the name Cattle Egret or its equivalent in many languages. Heatwole (1965) has convincingly demonstrated the biological advantages of A. ibis' associating with cattle. Birds feeding within one metre of a grazing beast obtain $1 \frac{1}{4}$ to $1 \frac{1}{2}$ times as much food and make $\frac{2}{3}$ as many steps getting it as do non-associated egrets, chiefly because grazing cattle disturb large numbers of insects which 


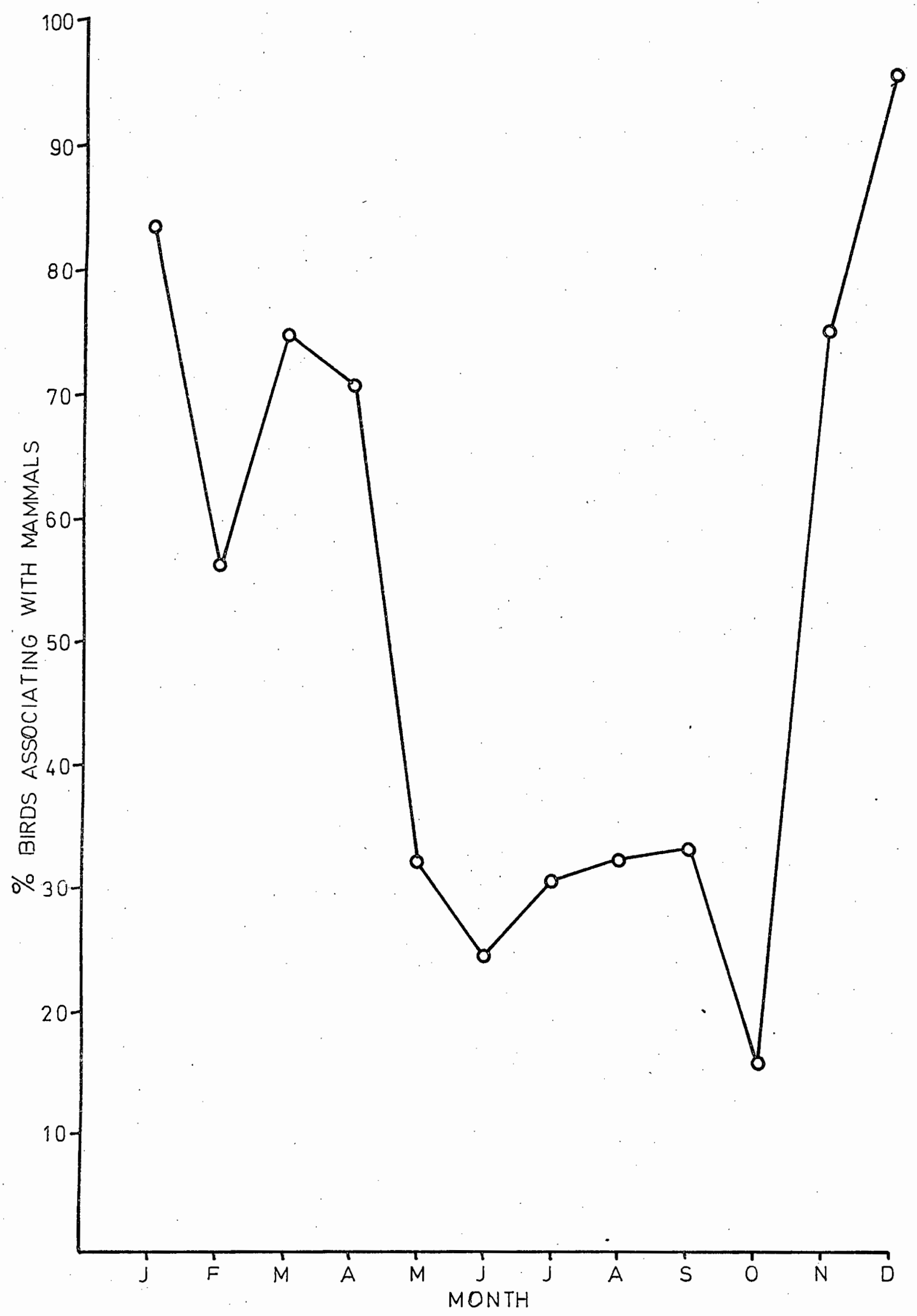

FIGURE 21. Monthly variations in percentage of birds associating with mammals. 


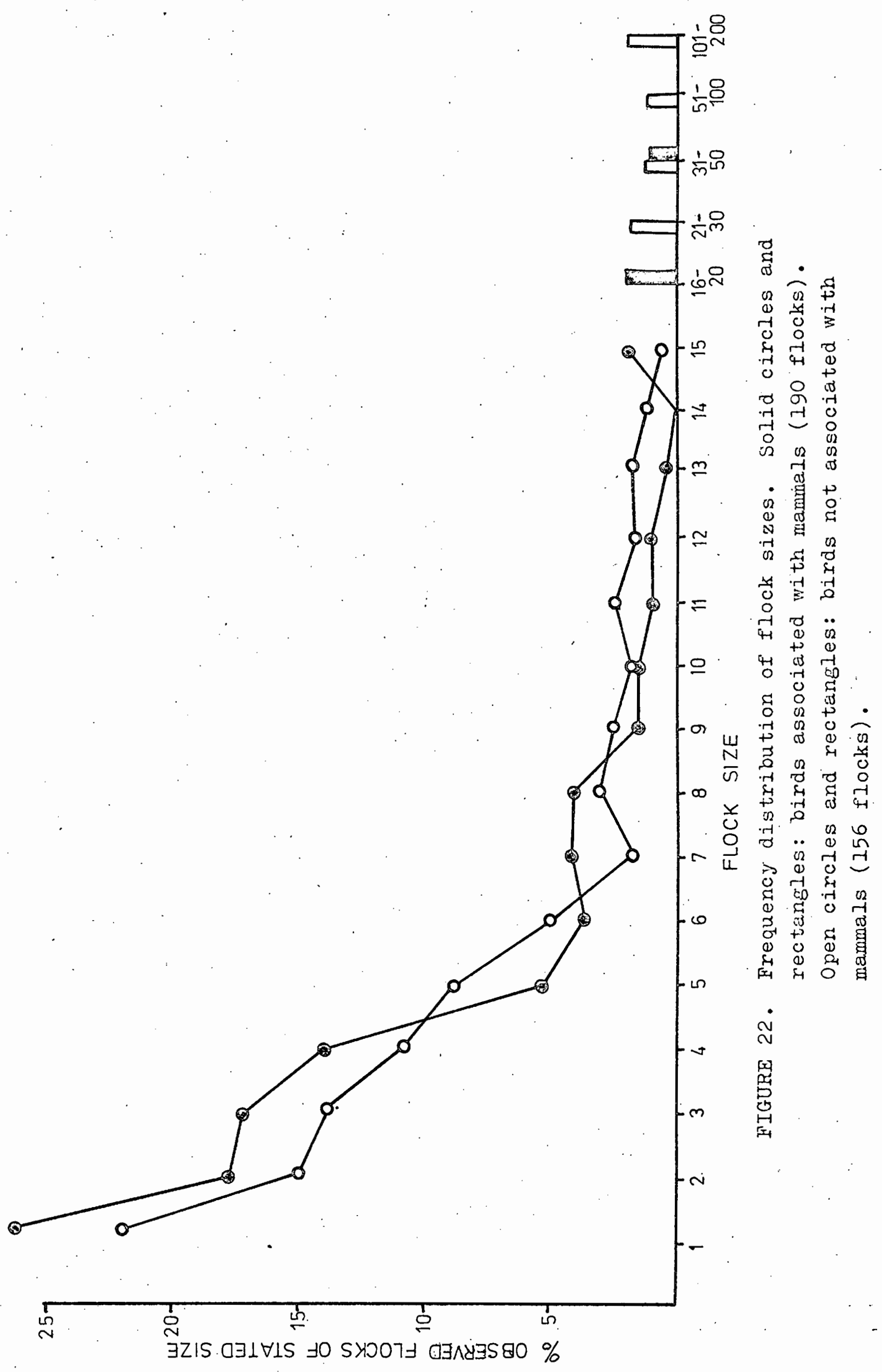


the birds would otherwise not have seen.

During travels in 1965-1967 all feeding A. ibis (total 2052), cattle, sheep and horses were noted; if a bird was feeding within three metres of a beast it was recorded as "associating". (Heatwole's standard of one metre was felt to be too strict). No attempt to standardise the travelled routes was made, so the monthly figures are not directly comparable. Nevertheless, the results (figure 21) show a marked tendency for A. ibis to associate with mammals more frequently in the ary summer months. The percentages associating vary from 16:8 (October) to $96.1 \%$ (December). These values are all certainly too high, owing to the tendency of an observer to look for birds around cattle and to overlook those not feeding in associated, but this is not important since it is relative values which are of interest.

On the basis of Heatwole's (1965) results, figure 21 suggests that food is harder to find and catch during the dry season. During the months June - September the countryside is covered with numerous small, shallow temporary marshes, and $21.2 \%$ of the A. ibis observed in these months were feeding in marshy ground. The species' habit of following ploughs to feed on the exposed insect larvae, etc. has probably been recently acquired (Heatwole 1965). 3.66\% of the 2052 birds observed were feeding in this manner.

Figure 22, derived from the same set of observations as figure 2I, gives the percentage distributions of flock sizes for both associating and non-associating birds. (Flocks are often loosely integrated but the term was extended. to include groups of birds where the minimum distance between individuals was a maximum of 20 metres.) Single birds occurred most frequently; the average flock size for associating birds was 4.24 ( 190 flocks) and 7.99 (156 flocks) for unassociated biras. Speculation on this point of difference is not justified here, for the flock sizes may be influenced by the host animals' behaviour and by ecological factors unknown to the author. Directly comparable figures for other species are not available,

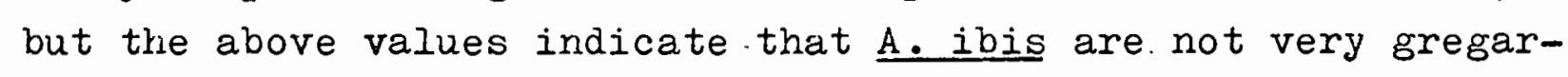
ious while feeding. Close flocks would be disadvantageous to an insectivorous bird, because the detection and capture of insects is likely to be inhibited by the prescence of other individuals. For instance, Crook (1964) found that seed-eating Ploceidae have much denser and closer co-ordinated flocks than insect-eaters do. Figure 23 shows that there is no great 


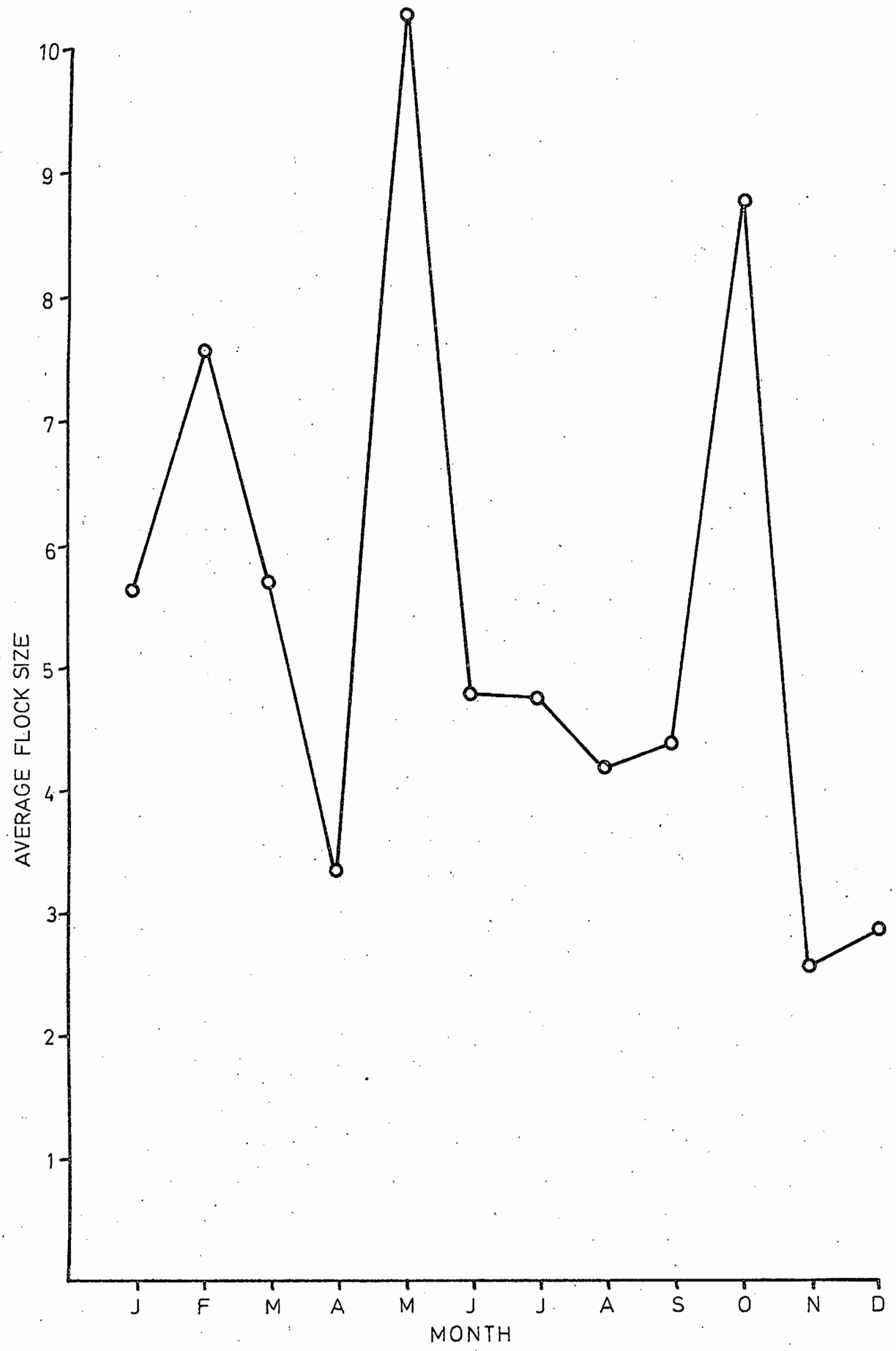

FIGURE 23. Monthly variations in average flock size. 
seasonal variation in average flock size. The May peak was chiefly caused by two very large flocks observed. in that month, and the October peak is probably an artefact resulting from a very small sample.

A. ibis has definite preferences for certain types of animals. The same set of observations used for figure 21 showed that an average of 0.183 A. ibis attended each cow seen ( 3378 beasts), 0.285 attended each horse and donkey (267 beasts) and 0.0084 attended each sheep ( 4344 beasts). (The differences are all statistically significant.) This preference for large: ungulates is also exhibited towards wild animals. Out of 8 publications consulted which mention A. ibis - wild animal relationsbips in Africa, there are 6 references to buffalo, 5 to elephant, 2 to rhino, and one each to "big game", hippo, giraffe, eland, zebra, waterbuck and "larger antelope". This indicates that the original hosts of A. ibis were pachyderms and larger Artiodactyls, particularly buffalo. (The present author can add Ostriches to the above Iist, and Jenkins and Ford (1960) record pigs, poultry and kangaroos in Australia). The habit of perching on cow's backs is farless common than the literature leads one to believe, and only $0.2 \%$ of all A. ibis observed were on a beast's back. Elephant and hippo are apparently much favoured however.

Several factors; the preference for buffalo among wild animals and for cattle among domestic stock, and Heatwole's (1965) demonstration of the biological significance of the bird mammal: relationship, all add up to suggest that the distribution of cattle may be a major factor determining the distribution of A.ibis. The possible influence of cattle on the species' spread in America has been investigated by Blaker (1967).

Briefly, A. ibis is capable of crossing the Atlantic unaided (from east to west: it is assisted by the N.E. Trades), so the surprising feature of the species' recent history is not that it reached America at all, but that its spread has been so recent (first South American record 1880). Ecologically suitable habitats have always existed in northern South America, so recent habitat changes in these areas cannot have played a dominant role in the species' initial establishment.

In pre-colonial Africa there were huge numbers of herbivores of over 100 species (Allen 1939), while South America possessed only 20 wild ungulates, mostly forest inhabitants (Cabrera 1960). The figure for North America was 22 (Niller and Kellogg 
1955). There is an approximate correlation between recent increases of cattle numbers and of A. ibis in Venezuela and Colombia; since 1900 there has been a fivefold increase in cattle to a total of about 25 million, in lands which were 400 years ago almost devoid of ungulates in open country. When other aspects of the species' biology (such as its preferences for cattle/buffalo, and the advantages of associating with them) are taken into account, the above correlation becomes significant. One could therefore perhaps conclude that the development of the cattle industry has promoted the spread of A. ibis in America.

Commensalism with large ungulates has recently become common in Egretta thula (Rice 1954) and has been reported for Casmerodius albus (Caldwell 1956), Florida caerulea (Howell in Palmer 1962) and Ardea cinerea (Curry-Lindahl 1956), so the potential may be widespread in the Ardeidae. Whether this is the case or not, Africa provides optimal conditions for evolution of the habit of associating with mammals. Post-glacial mammalian radiation reached its climax in Africa where millions of Bovidae once roamed. Apart from the Oxpeckers Buphagus (Attwell 1966) and vultures, no other birds have "used" the huge numbers of African herbivores to any extent, which suggests that A. ibis was in some way pre-adapted for commensalism. 


\section{BREEDING BEHAVIOUR}

\subsection{Colour- and piumage-changes}

Normally, A. ibis' beak, lores and iris are yellow to pale yellow, but for 10 - 20 days (approx.) before egglaying each year, these parts change colour in both sexes; the beak and iris to red and the lores to ruby-magenta. The legs, normally dark green, turn a dusky red. These colour-changes are associated with numerous behavioural changes, detailed in sections 3 and 5, all changes presumably having a common hormonal basis. The "breeding colours" of all three species studied are illustrated in the colour plate.

One to three days before an individual acquires a mate, the colours begin to fade. Firstly, the tip of the beak (in A. ibis) becomes yellow and a yellow ring appears around the pupil. Over a period of 5 - 10 days the redness recedes towards the base of the beak, and in the case of the eyes towards the perimeter of the iris. The legs slowly become darker again. In all observed pairs, the redness disappeared completely one to four days before the first egg was laid.

The most striking aspects are (a) the high degree of synchronisation between the sexes, and (b) the constancy of the colour-changes in relation to the day of pairing and the day of egg-laying. With regard to (a) and (b), there is however no way of knowing whether the changes follow a fixed hormonal cycle, or whether the redness-fading is determined by social factors, with mate-acquisition "switching off" the hormones which control redness.

Soft-part colour-changes associated with the early stages of the breeding cycle in Ardeidae, first recorded in the thirteenth century (Meiklejohn 1952), have so far been observed in at least 18 species. The only other Non-Passerine group in which soft-part colour-changes have been recorded is the Plataleidae (Blaker, in press).

It is mentioned above that redness always begins to fade shortly before a mate is acquired, and to a human observer an individual's situation in the breeding cycle can be quite accurately inferred from the degree of redness of its beak. It is therefore possible that the system of colour-changes provides a method whereby a bird can recognise whether a member of the oppo- 


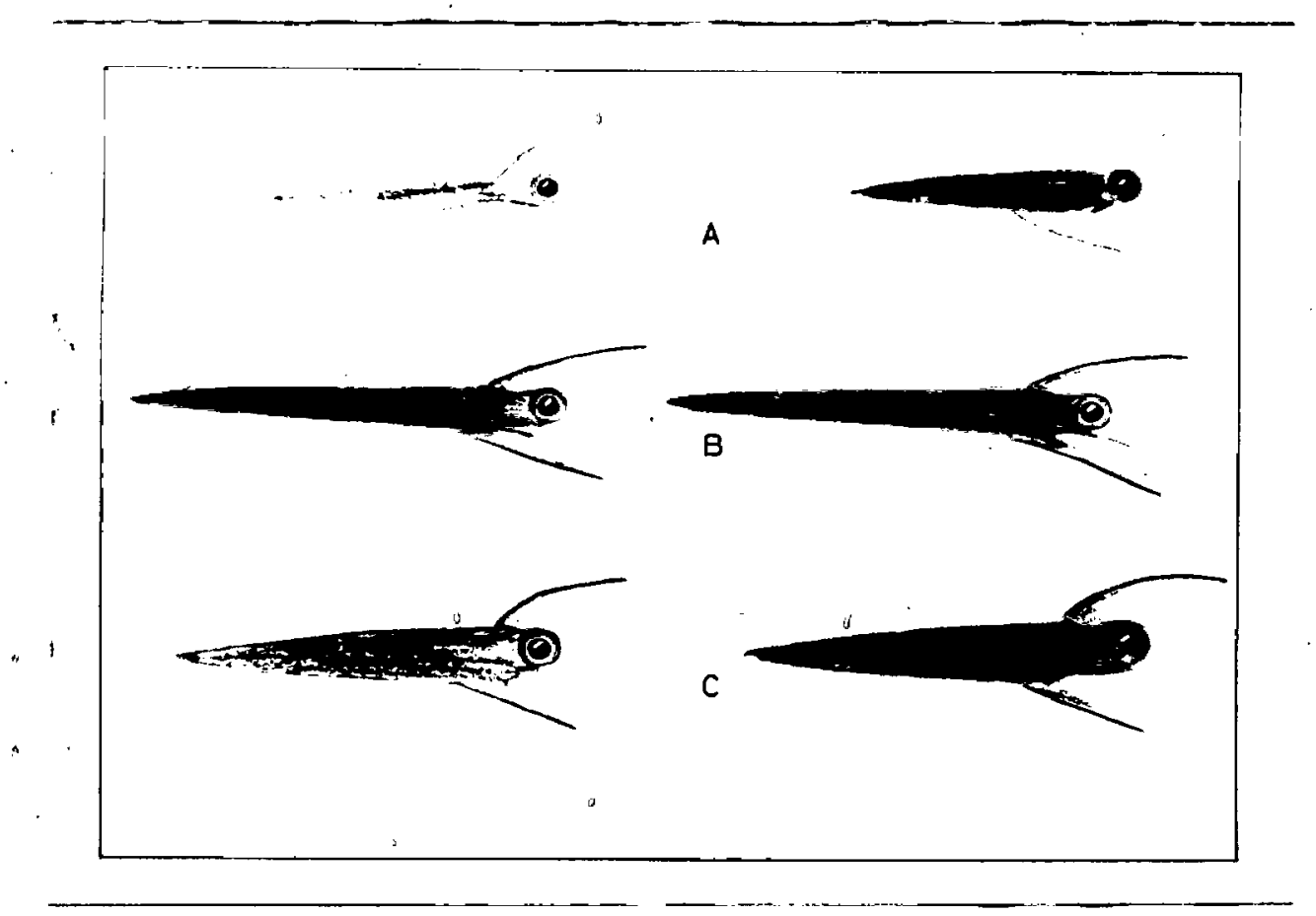

Colour-changes of beak, lores and iris in Ardeola ibis, Egretta garzetta and E. intermedia. Left, "non-breeding" colours. Right, "breeding" colours. 
site sex is ready to pair or not. In most species, sexual receptiveness is signalled by behavioural changes, but there is no reason why colour-changes should not be involved.

During the breeding season all adult A. ibis develop buffcoloured filamentous crest, pectoral and scapular plumes. The movements of the crest plumes have considerable importance in agonistic displays, as described in section 3.2. Erection of the pectoral and scapular plumes occurs in most displays. Complete rejoveal of the scapular plumes in one pair (both sexes) during the incubation period did not affect either bird's responses in the slightest; greeting cerenonies continued as normal. Scapular plumes are possibly therefore only functional during mate-selection and pair-formation.

\subsection{Colony Development}

The onset of the breeding season is marked by the arrival of a small group of males at the future nesting area at about $1500^{\circ}$ to 1700 hours one afternoon. In each of the two colonies whose development was followed in detail the initial nucleus was a group of trees several hundred metres from the nearest roosting area. These birds, which have fully developed plumes and red beaks, spend a few hours perched quietly in the treetops, returning to the roost at sunset. Incipient nest-building and stick-collecting movements are the only activities. The following afternoon, birds arrived earlier and in greater numbers and on the third afternoon or later, the phase described in section 5.3 begins and the birds remain overnight. Flap Flight Display, Twig-shake, Chatter and Forward Display, become common activities as each male defends its display site against females. Within 10 days, hundreds of red-beaked birds are arriving at the nesting area every afternoon, a large proportion of them non-vocal and inactive. It is possible that many of these individuals roost in the colony for a few nights before they reach a state of physiological readiness and become involved in the mate-selection process. Information on the numbers, age and occupation of non-breeders was not collected as this overlapped with another study. At the Paarl colony, Egretta garzetta, E. intermedia and Nycticorax nycticorax began breeding at the nesting area several days before the first A. ibis arrived. 
TABIE 5. Dates of commencement and completion of different breeding stages at Paarl colony, 1966.

\begin{tabular}{|l|c|}
\hline \multicolumn{1}{|c|}{ event } & date \\
\hline First A. ibis leaves roost and arrives & 20 or 21 August \\
at nesting area. & \\
First pair formed and first & 23 or 24 August \\
copulation & 30 or 31 August \\
First egg laid & 22 to 25 Sept. \\
First egg hatched & to 15 Nov. \\
Last egg laid & to 7 Dec. . \\
Last egg hatched & \\
\hline
\end{tabular}


Until nest-building has commenced, the birds at the colony are highly sensitive to interference and will desert the area, sometimes permanently, if there: is much human activity in the vicinity. Once the eggs have been laid, however, little short of nest-destruction will cause the birds to leave. It is highly unlikely that this change is due to habituation, but is probably an adaptive mechanism which helps ensure the reproductive success of the colony as a whole. If the first few birds were to tolerate the presence of predators in the area, heavy mortality could result at the egg and nesting stages, so tolerance has been strongly selected against.

Restlessness and group movements sometimes precede the onset of breeding. Skead (1966) mentions this, and to give one example, at the Rondevlei roost at 1805 on 20 August, 1966, about $80 \%$ of the A. ibis present flew up in a sudden "dread" or collective upflight (Iind 1963), circling the area twice in a co-ordinated group before settling again. Synchronised flights are rare in this species and may indicate periods of peak social awareness.

Table 5 lists the dates of commencement and completion of some of the breeding-cycle phases at the Paarl colony in 1966. At the Paarl, de Beers and Rondevlei colonies in 1965 and 1966 the dates of first egg-laying all occurred between the 12 th and 25th of August. Compared with many other gregarious species, the egg-laying period of 68 to 78 days in A. ibis (present study only) is very long.

Unlike many colonial birds, notably ground-nesting marine species, (1963) A. ibis nests are not built a more or less fixed distance from the nearest neighbour's, resulting in a uniformly dense colony. Instead, the inter-nest distance (between nest centres) at the time of building varies from 50 $\mathrm{cm}$. to 6 metres, with some new nests continuing to fill in the larger gaps for the entire duration of nest commencement. This contrasts strongly with the situation in, for example, Sula bassana, where $72 \%$ of inter-nest distances lie between 2 and 2.5 feet apart, and attempts to "squeeze in" new nests are accompanied by severe fighting (Nelson 1966a). The absence (in A. ibis) of this strong tendency to acquire a site as near as possible to others probably indicates relatively weak gregarious tendencies, as compared to Sula bassana 
FIGURE 24. Map of the Paarl nesting colony area.

$A-F$ : six stands of trees containing

A. ibis nests. I - 6: nest-material collecting areas.

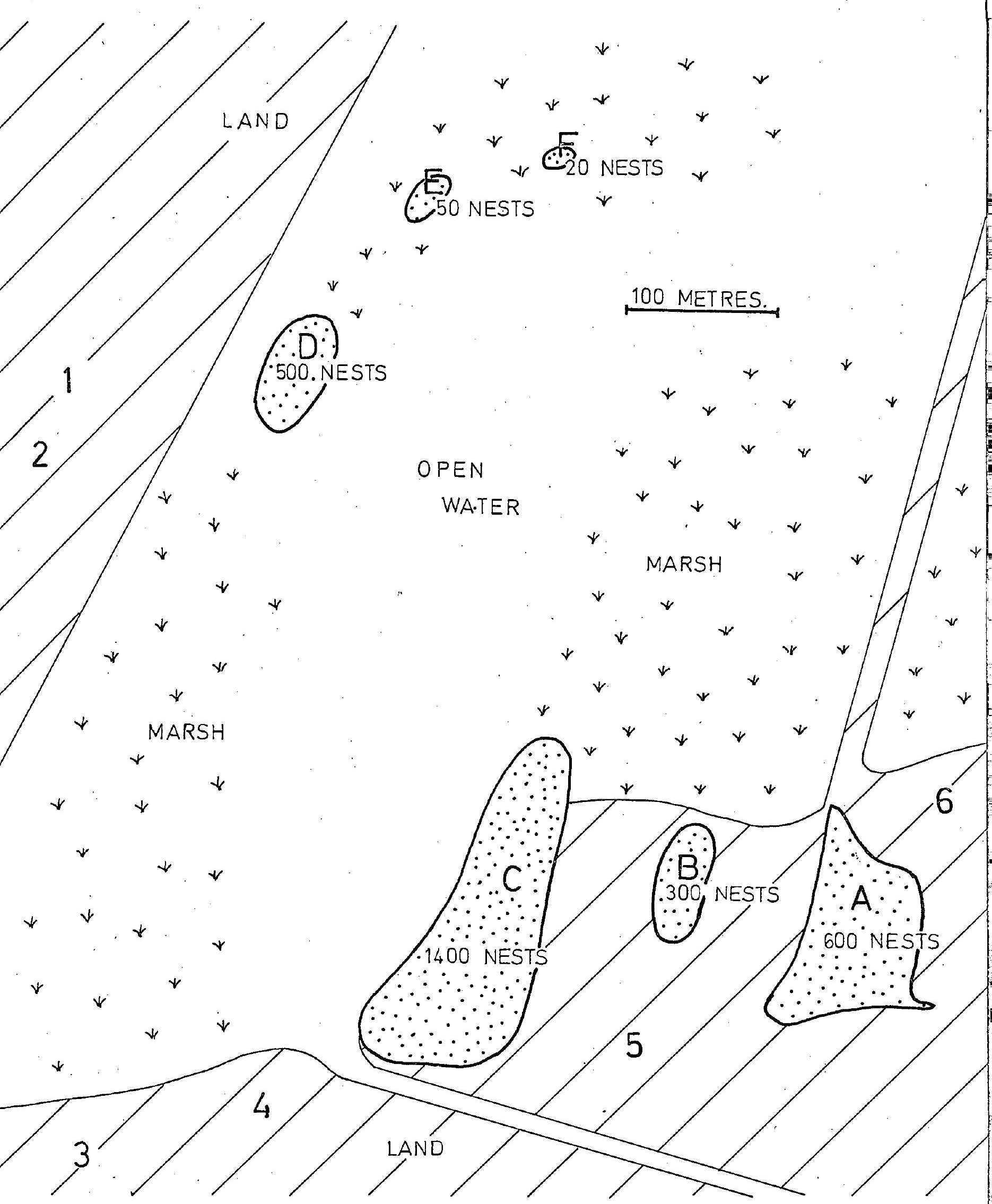


Development of the Paarl colony was complicated by the fact that the nests, ultimately 2,800 to 3,000 in number, were spread over six isolated groves of trees, separated by water and marshy ground. Figure 24 shows the distribution of the 6 nest areas, and the number of nests each contained. In 1966, the first A. ibis nest at $D$ was commenced on the 23 rd or 24 th August, at $C$ on 9 th September, at $A$ and $B$ on 12 th September, at $E$ on 12 th to 15th September, and at $F$ on lst to 4 th October. In 1964 and 1965 only areas A, B and C were used by the birds, and it is possible that human interference in these years caused the first-arrivals to choose area $D$ (an island) in 1966. Nonbreeding birds roosted in the northern half of area C until 20th August, 1966, when their numbers began to decline as birds moved to $D$ and became recruited into the breeding population. By late september few non-breeding birds still roosted in the trees at $C$ every night and nest-building began there. From early October onwards all non-breeding birds, those which had yet to breed and later, those which had completed the cycle, roosted at areas $A ; B, C$ and $D$.

A. ibis commonly breeds in mixed heronries with other Ardeidae; in the case of the Paarl colony, Egretta garzetta, (27 ne:ts) E. intermedia (14 nests) and Nycticorax nycticorax (approx. 40 nests), with Ardea cinerea, A. melanocephala and A. purpurea in the vicinity. The existence of mixed heronries is partly due to the fact that related species have similar habitat requirements, but the mutual attraction of nesting Ardeidae may serve a very definite function in some situations. A. ibis has never been found to nest in colonies of less than 7 nests in South Africa and colonies of less than 50 nests are unusual (Siegfried in litt.), which suggests that it, in common with many other gregarious species, is physiologically incapable of reproducing when present in very small numbers. It is easy to see that this could be a limiting factor at the edge of the species range, where densities are very low, by preventing new colonies from developing. It is: therefore: significant that at one extreme limit of A. ibis' rapidly-expanding range in North America, two pairs successfully bred in a mixed heron colony in Ontario. (Buerkle and Mansell 1963). Presumably, the birds' nesting-density requirements were satisfied by a form of stimulus-generalisation. The stimulus involved could not have been "white birds" as there were none present at the Canadian colony, so must have been "heron-like" birds. 


\subsection{Interactions within the pair}

The social behaviour of $A$. ibis at nesting colonies will be described separately in terms of intra- and extra-pair relationships, partly to simplify the classification of their behaviour. Following Johnsgard (1965), courtship has been divided into mate-selection (the interval between arrival at the colony and the moment of pairing) and pair-formation stages (the interval preceding laying of the first egg). Actual use of the word courtship' is avoided here because of its anthropomorphic overtones.

\subsection{Nate-selection stage}

The first males to arrive at the colony are relatively inactive for a few days, so it is assumed that throughout the breeding season each male visits the colony for a few days before it begins active displaying and attracts females. Although it is impossible to follow individuals for long, the continuous movements of many birds in a "new" colony presumably corresponds to the "flying around" phase in Butorides virescens males (Neyerriecks). Owing to the shyness of unmated males, it was possible to mark only three, so that where their movements are concerned it has been necessary to rely largely on impressions. The first individual paired 15 hours after it was marked, while the second was still unpaired after 25 hours and the third still unpaired after 49 hours. My overall impression was that the interval between adopting a territory and securing a mate is 3 or 4 days (also in skead 1966).

For these few days before pairing, each male occupies a small territory in the colony, displaying on it and vigorously defending against intruders an area of two or three metres radius. It is usual (section 5.32) for this territory to be abandoned and another nest used for nesting after a mate has been acquired. Only an estimated 10\% of unmated males adopted a disused nest as a territory, in contrast to Butorides virescens where the male always uses an old nest if one is available (Meyerriecks). For the reason stated above, figures on the movements of unmated males are too. scanty to be meaningful but they definitely do not roam around the colony as much as do unmated male Egretta garzetta and E. intermedia (this study). When they are not interacting, and particularly during the early stages of pair formation, both sexes are inactive for most of 

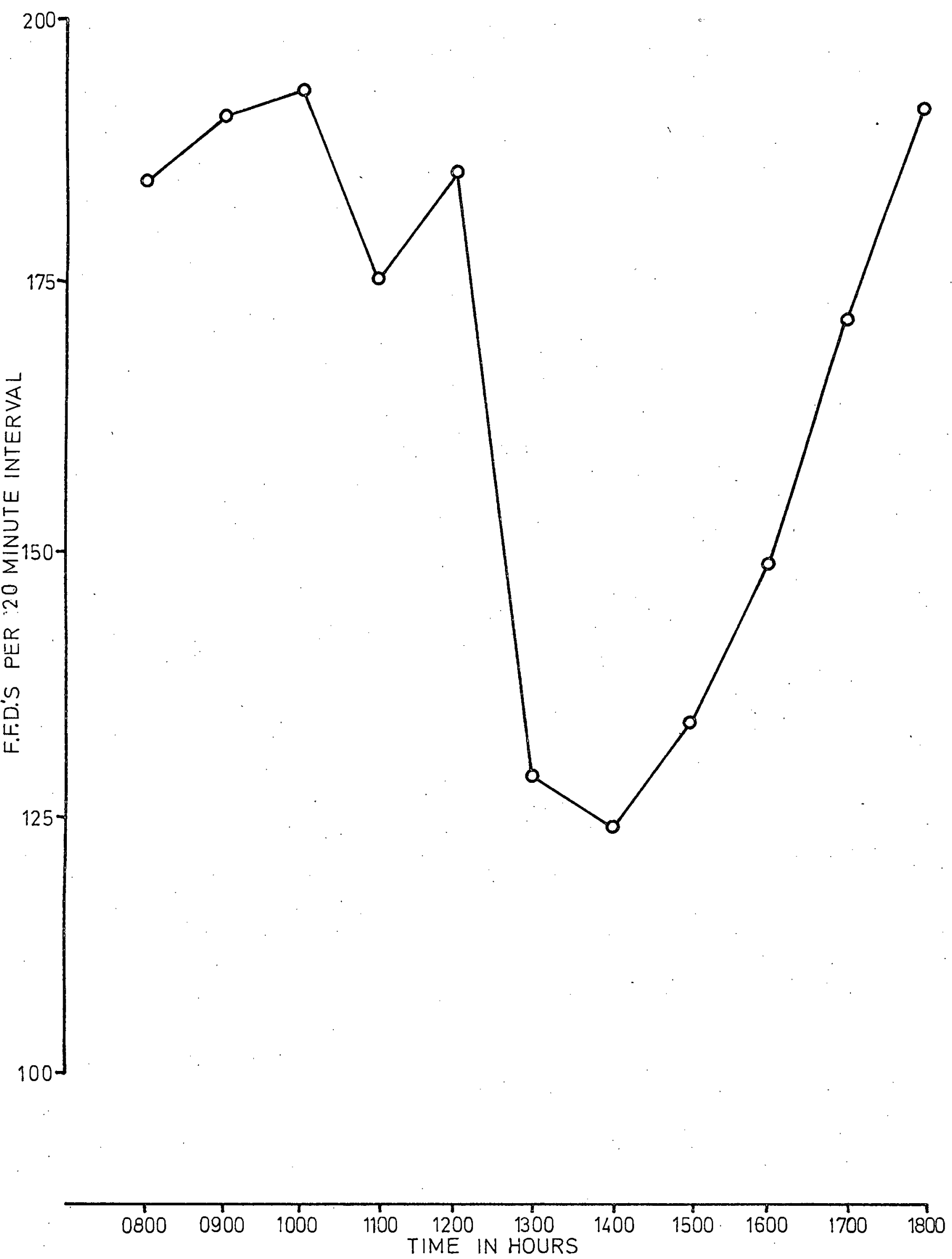

FIGURE 25. Hourly frequencies of Flap Flight Display. ixplanation in text. 
the time, preening, resting or sleeping.

As a conspicuous activity characteristic of unpaired birds, Flap Flight Display provides a useful index of the hourly and daily activity levels of these birds. Accordingly, the number of these displays heard per 20 continuous minutes each hour between 0800 and 1700 were recorded one day (18.9.66) in area $c$, which contained at the time about 300 birds, mostly unmated. In order to reduce the effect of weather as a variabie a warm, windless, cloudless day was chosen. Figure 25 shows that the morning level of activity is followed by a slight lull at 1300 - 1400 hours, with a definite peak in the late afternoon. Incidental to this, figure indicates a display frequency of about 1.6 F.F.D. per bird per hour, considerably lower than the values in table 1 , mainly because not all the birds are active at any one time. IHy overall impression was that there is little activity before 0800 hours in the morning, a slight peak at 1000 - 1100, with peak activity among unmated birds occurring between one and two hours: before sunset.

The commonest male displays are hoarse Rick-rack; Nasal Chatier, usually associated with Twig Shake; Flap Flight Display and wing spread. In addition to these displays, already described in detail, the males perform a number of very characteristic activities which appear little ritualised and cannot therefore be classes as displays, despite the arbitrariness of the distinction (introduction to section 3). The usual attitude of unmated males is hunchea, sometimes almost horizontal (figure 26) with neck retracted and beak well below the horizontel, so that the bird appears to be looking downwards. (This is a component of Forward Display). There is marked overall feather erection, the scapular plumes being particularly conspicuous as a result of the bird's hunched attitude. Normally, the wings droop very slightly; this may be an aggressive component (cf. Forward. Display). A very common movement is sideways swaying, the bird rhythrically shifting its weight slichtly from one foot to the other. The movement commonly occurs in association with Twis̆ Shake, is probably too inconspicuovs to have an important signalifunction. The male spencs much time slowly walking up and down and turning around. on its perch, frequently with Wing Spread and hoarse Rick-rack, all movements having a slow, deliberate appearance. To the humen eye, the foregoing movements are more characteristic of unmated males than any of their hi fhly ritualised activities are, 
so probably constitute the chief sex-recognition feature for A. ibis females.

Females are strongly attracted to males behaving in this manner, and one to six may be perched within a 5 metre radius of an actively displaying male. (This does not indicate an unbalanced sex ratio, but reflects the fact that all males are not equally active at all times). Not yet confined to a territory, the females fly from one male to another, spending a few minutes to several hours near each one before flyirig to another which is displaying more conspicuously. Characteristically, females perch on a level one or two metres above the male. Owing to the female's high mobility, it was possible to mark only one, an individual which appeared intermittently near the same male for 15 hours before the two became mated. Females appeared to spend less of their time at the colony than did males.

The behaviour of unmated females is much simpler than that of males. While watching or "attending" males, females have a highly characteristic long-necked. "peering" attitude (figure 26) with crest feathers partly erected and neck almost fully extended, even when it is horizontal. Their skeletal attitude is very similar to Alert, usually associated with strong fear tendencies, while the crest positions (usually 5, figure 3) indicate moderate fear and aggressive tendencies. In view of the birds' strong attraction to males and their subsequent behaviour, the crest position seems to be the more reliable teridency indicatcr. These females, which are completely non-vocal, continuously crane and peer at active males, giving an appearance of intense curiosity. (This anthropomorphic interpretation may in fact be correct for tame fledglings examining unfamiliar objects behave very similarly). They are clearly attracted by the male and not by its site, for they will follow a male if it moves its position. A male's response to its attending female(s) is invariably aggressive for peering females are repeatedly threatened with full intensity Forward Displays having the "rolk" call characteristic of the red-beaked phase. The threshold for Forward Display is very low at this stage for it is sometimes given in the complete absence of any trespassers.

A few interesting features emerge when comparisons with other Ardeidae are made as this stage. Firstly, A. ibis males have no activity corresponding to the croaking advertising calls 
of most other species studied so far, namely Ardea cinerea (Verwey 1930), Butorides virescens, Dichromanassa rufescens, Egretta thula (Meyerriecks) and E. garzetta (this study). Flap Flight Display and Nasal Chatter are probably the chief advertising displays in A. ibis. No reason is suggested for this difference, which presumably indicates no close evolutionary relationship between A. ibis and the above species. Stretch, although highly ritualised, is unimportant as an advertising display, for it is inconspicuous and quiet. Secondly, A. ibis males never ferform the long Circuit Flights or Circle Flight Displays (Mieyerriecks) recorded for Butorides virescens, Dichromanassa rufescens (Neyerriecks), Egretta garzetta and E. intermedia (this study). This must be due to relatively stronger site-attachment tendencies in A. ibis.

Although numerical proof is lacking, it appeared obvious $\therefore$ at the male's aggressiveness stimulates the female's interest, and that a male's threats and supplanting attacks very rarely drive a female away completely (also in Skead 1966); the converse * ine normal situation. It is very remarkable that Forward isplay's effect should switch from distance-increasing to distance-reducing (Tinbergen 1959) in some situations, so a digression is in order. The "rolk" call note is soft and mufflis and were it not for the movements which accompany it, would not be recognised as a form of the harsh "raa" of normal Forward Display. In the description of Stretch Display the r. rivile that displays with contrasting appearance have con-

sing functions was developed. It is very likely that this principle also applies to Forward Display, and that selection originally favoured males with modified "raa" notes by making the display less intensely distance-increasing, thus enabling the birds to pair more rapidly. The predominance of aggressive tendencies in the first place is of course another problem, and is dealt with below. Incidentally, the subject provides an excellent example of how the cause and effect of a single activity may be completely opposed in some circumstances.

For the 24-hour period before a male finally accepts a mate, the attraction of the females for the male increases markediy and they repeatedly fly (with Flap Flight Display) towards the male, only to be immediately threatened and repelled. The motivation of these females is interesting. During and -Lidiately before an approach the female's crest and neck feathers are fully erected and the beak slightly opened, indicating a 
sudden upsurge of aggressive tendencies.

This is borne out by the bird's subsequent behaviour, for when it eventually succeeds in landing on the male's back, with crest still fully erect, it performs a few high-intensity Back Bites before it is ariven off by the male with wild wing-flapping. by both birds. The female's behaviour cannot be a reversed copulatory attitude representing strong sexual tendencies, for the above points indicate that while on the male's back its motivation is predominantly aggressive.

A very noticeable feature of these interactions is the almost complete lack of intra-sexual aggression. Only twice has an unpaired female been seen to supplant another which had been peering at the same male. In both cases the birds concerned were about two metres apart, well outside each other's normal individual distances, so the supplanting female may have recognised the other as a potential competitor. Unpaired males clearly behave more aggressively towards unpaired females than to each other, (Skead (1966) emphasises the same point), and there is no doubt that male A. ibis do not aggressively compete among each other for females. Some form of sexual selection must, of course, operate in males.

For several hours before a male iinally pairs, interactions between it and the surrounding female(s) become more frequent and intense. There is also a change in the nature of these encounters, for the female begins to remain for longer periods on the male's back, eventually up to 20 seconds before it is chased from the territory, although the situation is always highly unstable and a sudden move by either bird results in the female being attacked. The male responds by crouching, but its crest and neck feathers are fully erect, so that the crouch cannot represent submissiveness or sexual tendencies. These changes in the male's behaviour are probably due to both cycliaal hormonal changes and to a summation effect of the female's approaches. Eventually one female is not attacked when it approaches and the birds are said to be paired. The description continues in section 5.22 .

The most outstanding features of interactions between the unpaired A. ibis are firstly the absence of sexual chasing males, and secondly the contrast between the territoricil agrressiveness of males towards females as compared with the persistent attempts of the females to approach potential mates as closely as possible. It is cormon for aggressive tendencies to predominate at the beginning of courtship (Tinbergen i952) but in A. ibis the male 
shows sustained aggressiveness in the face of female receptivity. This is the direct opposite to the situation in the great majority of animals. In most groups, the male actively pursues potential mates and is characterised by overt sexual and aggressive behaviour, while the initial response of the female is to flee (e.g. Mayr 1966, p. 95, p. 101). This type of situation is so familiar in both birds and mammals that it is unnecessary to quote evidence.

Forwardness in unpaired females and territorial aggressiveness in unpaired males occurs in many of the species which have been studied in any detail so far: Ardea cinerea (Verwey 1930), A. herodias (Cottrille and Cottrille 1958), Dichromanassa rufescens, Eoretta thula (Meyerriecks) Ardea melanocephala (North 1963), Florida caerulea (Meanly 1955), Ardeola ibis, Egretta garzetta and E. intermedia (this study). A search of the literature produced only one other species which has a mateselection system similar to that described above, the stork, Ieptoptilos crumeniferus (Kahl 1966).

It is necessary to explain (a) why overt aggressive behaviour has been selected as the normal method of advertising in some Ardeidae, and (b) why females are more receptive than males. Verwey (1930), referring to Ardea cinerea, suggests that because the male is solitary for most of the year, aggressive tendencies predominate in unmated males. This is almost certainly correct, but does not explain why females are far more sexually motivated and responsive than males are. The most likely explanation is that earlier in the families' evolutionary history, females always reached seasonal sexual maturity before males, which were consequently aggressive and maintained an indivioual distance despite the female's sexual interest in them. In this way, only aggressive behaviour would have been "available" for ritualisation, and selection by the receptive females would have caused overtly aggressive behaviour to acquire advertising and stimulatory functions.

One final point of interest is the apparent purposiveness of females which wander around the colony and congregate around displaying males. This situation is fairly well described by the definition of appetitive behaviour: "the flexible or variable introductory phase of an instinctive behaviour pattern or sequence" (Thorpe $1956 \mathrm{p} .3 \mathrm{I}$ ), the classical example/which is hunting by a predator. 
The first 10 minutes of the pair relationship are characterised by a continued high level of aggressiveness in both birds. As-described in section 5.22 , pair-formation is initiated by the fernale flying (Flap Flight Display) or running ( 1 case out of 6 ) onto the male's back. Both birds have crests fully erect which denotes agoressiveness, although the male crouches slishtly. Back-biting, at first performed by the female only, but after 20 to 60 seconds by both birds, is the commonest activity for the first hour or more. The actual biting movements, directed at head and neck, are little removed from overt attack and the beaks are wide open.

Detailed time-scores for the first 40 minutes of the pair relationship were obtained for two pairs, since considerabie importance is usually attached to the behaviour changes which occur immediately after the moment of pairing. These timescores (figure 27) illustrate the high frequency of Back bite in the first 5 minutes (average 5.8 displays per minute for males, 4.4 for females), which thereafter decreases rapidly in females and less so in males. After 10 to 20 minutes, the male's continued Back Biting begins to elicit signs of conflict in the female, with Head Flick and the crouch component of Stretch appearing. Within 19 minutes in the case of pair $V$ and 10 minutes in pair $S$, the male resurned Flap Flight Display. In pair $V$, the first nesting material was brought (aetails in. section 5.5) at:22 minutes and the first copulation occurred at 53 minutes. In pair $S$ the male attempted to mount the female at 14 minutes but no building or copulation occurred in the first 40 minutes.

After an hour the display frequency has fallen greatly, with the female perched quietly on the site with crest positions 1 or 4 (figure 3) but the male still showing signs of aggressiveness. A few mutual Back Bites occur, but are far lower in intersity than at the start of the pair relationship. While the pair are perching side by side, the displaying bird usually puts its neck across its mate's back and briefly nibbles the wing coverts on the other side.

It is evident from the above description that marked motivational changes occur immediately before and after the moment of pairing, so it is worthwhile to clarify these changes and to speculate on the mechanisms involved. Before the moment of 
FIGURE 27. Time scores for the first 40 minutes of the pair relationship in two pairs (explanation in text)。

$B$ : Back Biting

C: copulation

F: Flap Flight Display

HF: Head-flick

TS: Twig Shake

W: Wing-touch

Pàir S

$\sigma^{7}$

0859

$\frac{}{\text { BBBB BBB B BB B B B B }}$
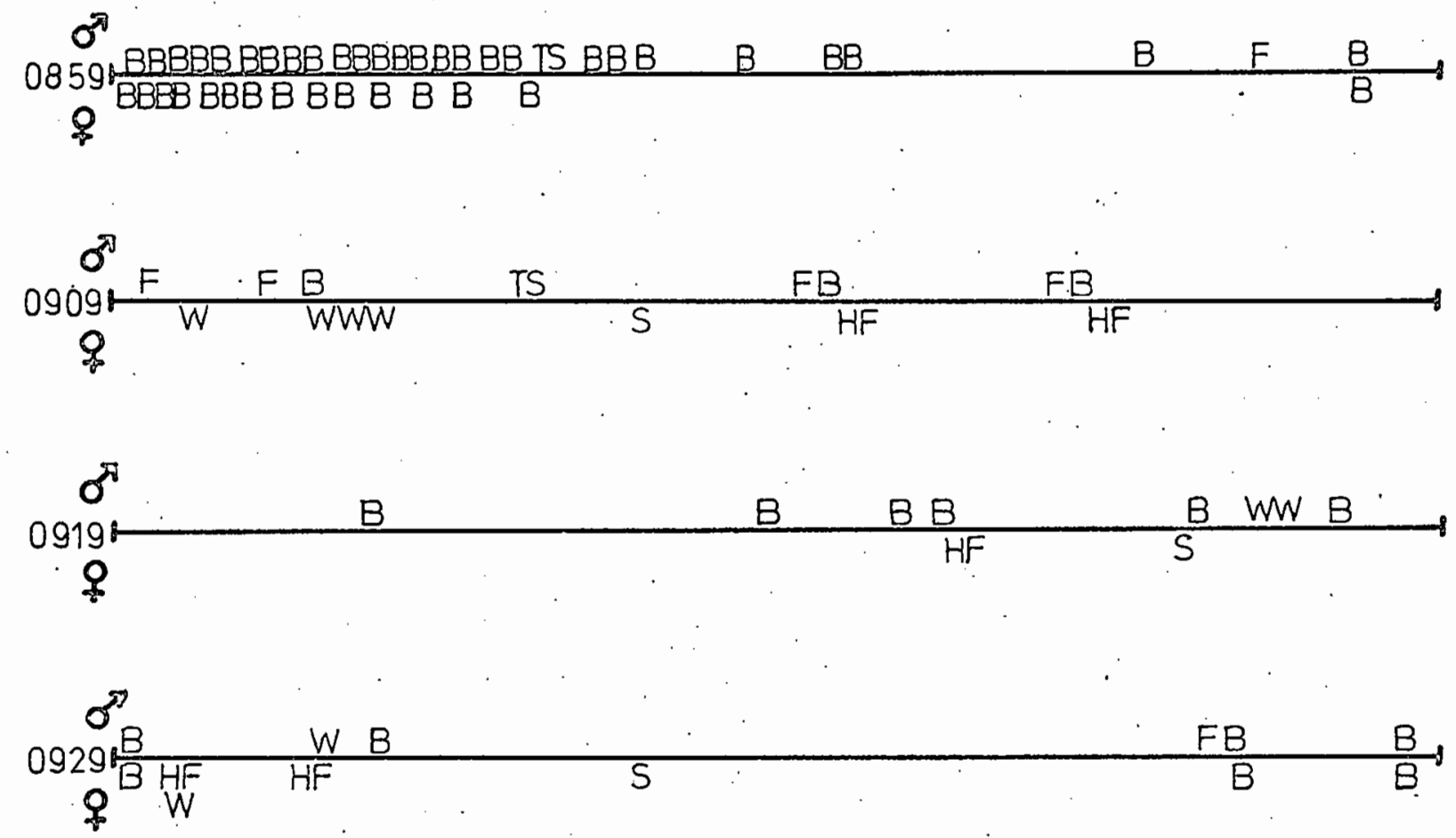

Pair V
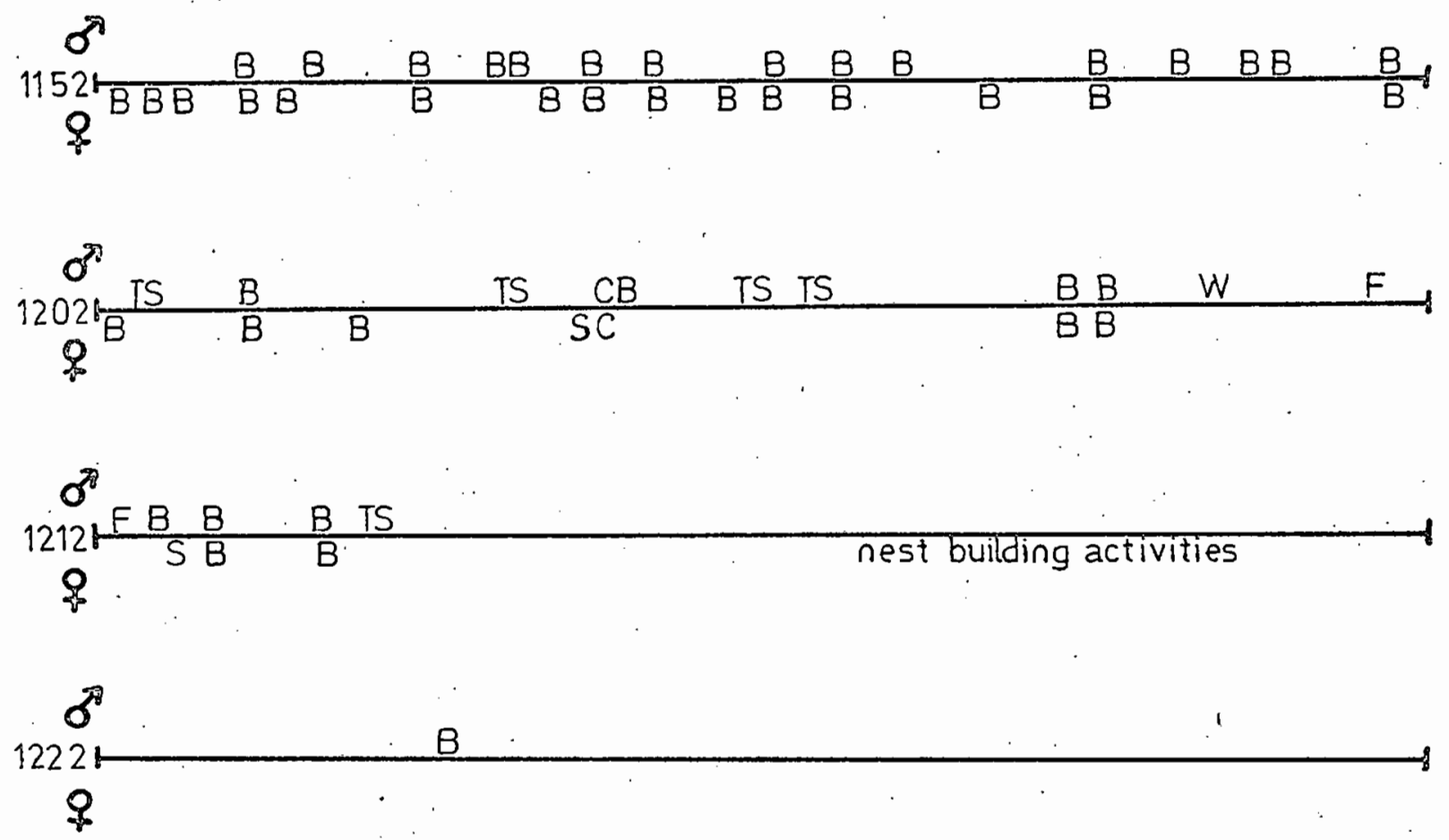


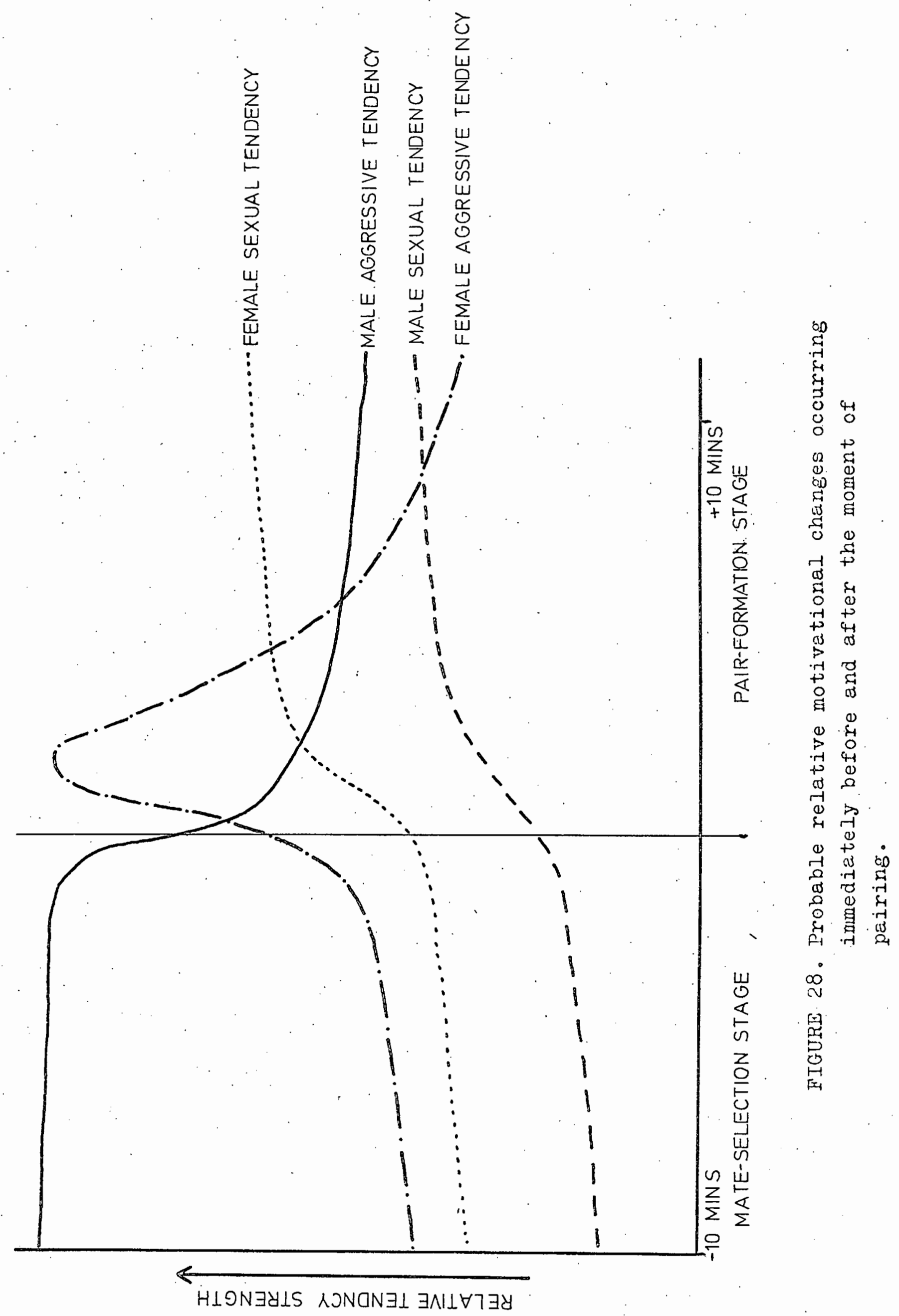


pairing the male is characterised by strong territorial aggressiveness. Females are attracted to these males, but fear tendencies must be present, otherwise they would not hesitate before approaching the male. Commonly stimulated by a male Flap Flight Display, the female approaches the male and lands on his back. (Reasons are given in section 5.21 why this does not represent reversed mounting). Simultaneously, the female's aggressive tendencies increase markediy, the crest, scapulars and neck feathers becoming fully erected. The male responds by crouching slightly and alloweing the female to remain on his back, but his level of aggressiveness is still high and for a few seconds there is delicate balance between overt aggressiveness and acceptance of the female. If the female is allowed to remain, a few minutes of aggressively-motivated Back Biting by both birds follows. Sexual tendencies are presumably present, particularly in females, which begin to give low-intensity stretch displays witrin 20 minutes. A steady decline of agoressiveness in both sexes follows, and copulation occurs within an hour. The most significant change to occur at pairing is the male's sudden and complete conversion from a "distance" to a "contact" animal (Hediger.1950) as regards his mate. This contrasts with the situation in Butorides virescens where the male, although aggressive, typically avoids the female after she has gained admittance to the nest. (Meyerriecks).

Constructed on the basis of all the above evidence, figure 28 illustrates the probable changes in sexual and ackgressive tendencies in both sexes. It is tempting to assume that the sudden decline in male aggression is due to intimiation caused by the female's aggressive approach, but internal changes affecting aggression must take place in the male, because the successful female's behaviour towards the male does not appear to differ in character from the numerous unsuccessful ones which preceded it.

Once a pair have renained together for a few hours the relationship has stabilised, so the phase named here "Pair formation" could in fact be called "Pair consolidation". Most of the bird's time is spent perched on the nest site (figures on attentiveness appear in section 5.8), with little activity other than nest-building activities and greeting ceremonies. Three points are useful. in sex identification. In 132 minutes' close observation on three pairs at this stage, the f'emale's head was on a lower plane than the male's for 120 minutes, which probably 
indicates male dominance, as it is quite common for the more dominant of two animals to hold its head higher than the other's (e.g. Sauer and Sauer 1966). A female: spendsfar more time arranging nest material than does the male. Lastly, feathererection is usually more marked in the male.

Within a few hours of pairing, the birds usually abandon their site, the male initiating the move and flying off, with the female following. In ten out of the eleven pairs in which the moment of pair formation was observed the site was abandoned the following day, although the observer's presence may have been a factor in some of these cases. Less than a day after the arrival of the first birds at area $C$ (section 5.2), several pairs were noticed. The interval between arrival at the colony and obtaining a mate has been estimated to be three or more days, these birds must have paired at area $D$ before moving to area $C$ for nesting. After pairing, the male's defended area becomes noticeably smaller. In 1965, 6 pairs were colour-. ringed in addition to being sprayed with dye. None of these birds was seen in 1966, although an intensive search was made in their 1965 nesting area. Although the sample is small, one can assume on this basis that individuals do not return to the same nest sites in successive years, and as a corollary that they do not retain the same mates from one year to the next.

Nest building begins within a few hours of pairing, although at least a day passes before the nest becomes "noticeable". The interval between commencement of nest building and laying of the first egg averaged 7.3 days in 6 pairs with a range of 5 to 10 days. Commonest displays throughout the pair formation stage are Back Biting (often with Soft Chatter), Stretch (female only) and hoarse Rick-rack. A striking feature of the behaviour of A. ibis pairs at this stage, as compared with the Egretta species (this study), is the much higher incidence of close body contact within A. ibis pairs, which may spend long periods perched side by side. As described in section 3, greeting ceremonies in A. ibis always involve several seconds close body contact, which contrasts noticeably with greeting in Egretta. Functionally, the difference may indicate more efficient pair-bond maintaining mechanisms in A. ibis. The most conspicuous activity during the pair formation phase is nest building, described in detail in section 5.5. $99 \%$ of the nest material is collectea by the male and brought to the female who actually builds the nest. 
After the first day of pair formation, there is normally no evidence of intra-pair aggressiveness, but exceptionally situations arise which cause the male to attack the female. In one such case, female A threatened a trespasser, was threatened in return, and a fight developed. Male A then flew down from a perch in the treetop, drove the trespasser from the site, then momentarily redirected his attack against his mate, but within a second their behaviour switched to high-intensity mutual Back Biting. Incidents such as these are rare and not comparable to the sustained intra-pair aggression which occurs, for example, in the Gannet Sula bassana (Nelson 1965).

\subsection{Copulation}

Copulation: in A. ibis is seldom preceded by displaying by either partner. In the minority (30\%) of cases the male, perched nearby, was stimulated to approach the female when she gave a low-intensity Stretch Display on the nest. The components described in section 3.2 , include downward-squatting movement, upward-pointing beak, flattened crest and usually a soft "roo" call. This comprises female soliciting; the exact significance of the above components are discussed in section 3.2. In the remainder of cases $(70 \%)$ the male, which had been perched inactive near the nest, slowly walked towards the female on the nest, while giving a few Rick-racks. The female responds to the male's approach by crouching on the nest, with wings slightly spread and the angle between tibiotarsus and tarsometatarsus between $20^{\circ}-80^{\circ}$. (The significance of this is discussed in section 7.20). The male then steps onto his mate's back, grasps the humerous region of each wing in his feet, begins to perform rhythmic treading movements which gradually accelerate, then lowers his tail to achieve cloacal contact and simultaneously begins to flap in order to retain balance. In $16 \%$ of 25 copulations the male grasped the female's nape in his beak. After dismounting, they both perform body shakes then preen, usually paying no further attention to each other. Soliciting by the female does not always lead to copulation; sometimes the male shows signs of conflict, giving Head-flicks and lowintensity Back-Bites.

Copulation has only been observed on the nest or within $50 \mathrm{~cm}$. of the rim, but males occasionally mount other females on adjacent nests (section 5.92). The reversed mountings mentioned 


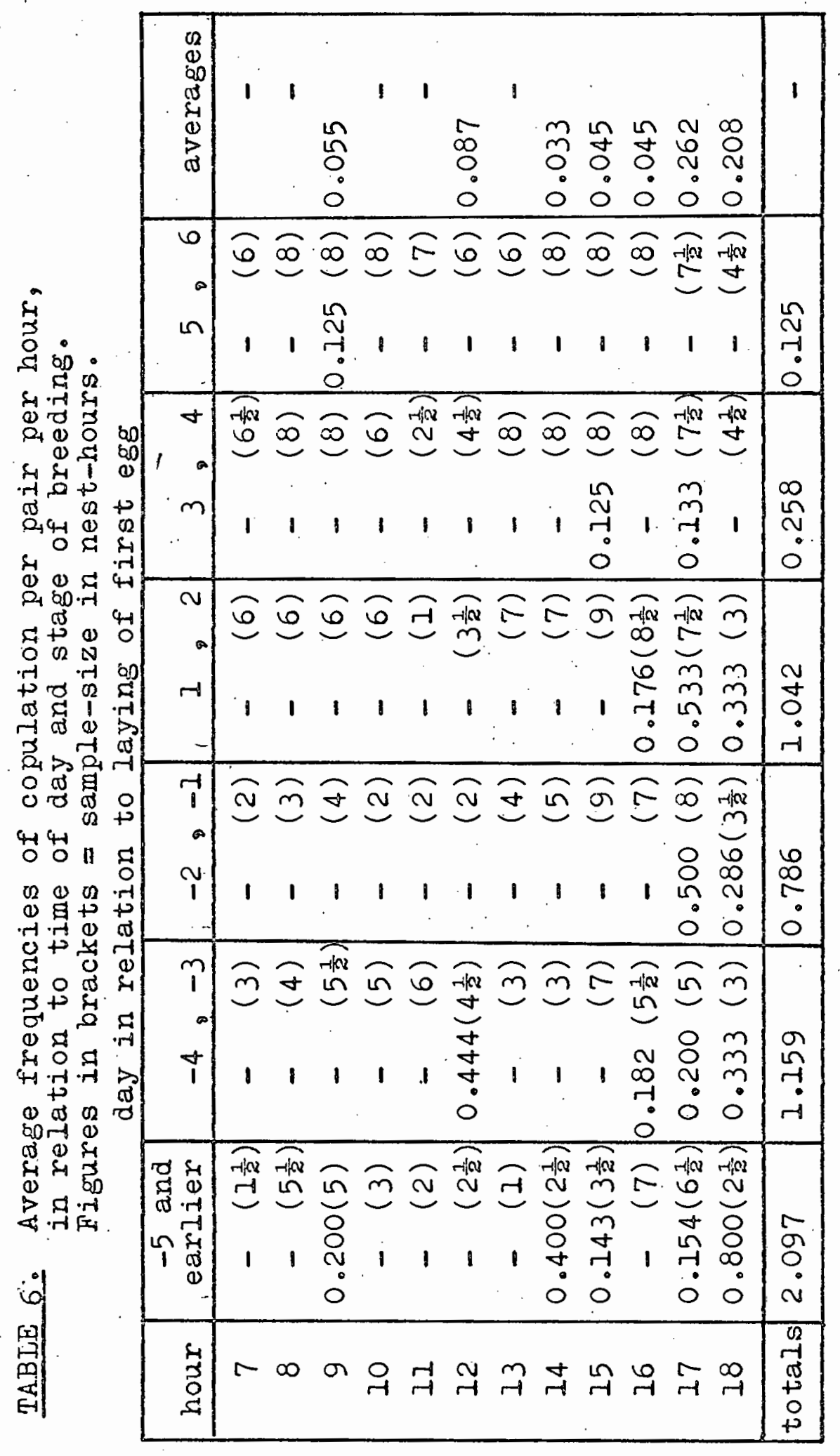


by Skead (1966) are aggressively anà not sexually motivated (section 5.31). The most striking feature of copulation in A. ibis is the low frequency and intensity of accompanying displays, which suggests that mutual inhibitions against body contact are minimal. Frequently a male mounts his mate and treads for a few seconds without achieving cloacal contact (2l incomplete as compared to 25 normal copulations over the same observation period). The majority (62\%) of these incomplete copulations were preceded by females soliciting, implying that sexual motivation is relatively stronger in females. Supporting this contention that females are more receptive are the observations that males never attempt to force copulation on their mates and that males soinetimes show conflict behaviour at their mates soliciting. In addition, males frequently show signs of conflict in response to female soliciting.

Table 6 gives the frequency of copulations per hour in relation to time of day and stage of breeding (observations on 12 nests). The great majority occur between 1630 and 1830, although many may have taken place after formal observation ended at 1830. This high degree of temporal synchronisation must have a mutual sexual stimulatory effect of the type visualised by Darling (1938). Table 6 shows that prior to egg-laying each pair copulates about 0.79 to 1.16 times per day and two or more times on the day they become mated. As some copulation occurs outside of observation hours (0630 1830) these figures are probably 10 to $30 \%$ to low. Even so, each jair of A. ibis probably does not copulate more than a total of 20 times. The daily frequencies fall rapidly after egg laying, and copulation has not been observed later than 6 days after the first egg has been laid. Copulation in A. ibis is considerably less frequent than in other species for which figures and first-hand comparisons are available, such as Ardea melanocephala (Skead 1966 and pers. obs.) or Sula bassana where individual frequencies of up to 4 per 40 minutes are "not unduly high for sea birds" (Nelson 1965). No reason car be seen for the very low rate in A. ibis, particularly as the species is little affected by predators and is therefore not especially vulnerable during copulation. However, no other factor favouring low frequency of copulation can be visualised. 


\subsection{Nest-building}

5.51 Introduction

$99 \%$ of the nest building in A. ibis is carried out by females, while $96 \%$ of nest material is: collected by males (based on 637 observed nest-material collecting trips). This division of labour between the sexes may be widespread in the Ardeidae as it has: älso" been recorded in Hydranassa tricolor (Huxley in Bent 1926) and Florida caerulea (Meanley 1955). This system may have arisen because (in $\underline{A}$. ibis at least). if both sexes collected material the nest would be unguarded at times, which would inevitably result in it being destroyed by other birds (details below). A. ibis nests at Paarl were constructed almost entirely out of dry, brittle sticks and weed-stems, ranging fros about 8 to $90 \mathrm{~cm}$. long; leaves, pliable twigs and grass are not often used. Nest material is always brought to the nest one item at a time. Nest-building begins within an hour of pairing but it is usual (section 5.32) for the first few sticks to be abandoned and for the male to chose a new site. Figure 29 shows that nest-building continues throughout incubation with decreasing frequency, and is inhibited almost completely when the young hatch; an adaptive feature since chicks could easily be damaged by nest-building activities. Three nests taken apart at the time the eggs were due to hatch contained 180,263 and 292 sticks respectively. The interval between pair-formation and laying is 7.3 days (section 5.32 ).

\subsection{Techniques}

Ardeid nests and nest-construction techniques are extremely simple. The basic building movement, "tremble-shove" (Lorenz 1955 in Neyerriecks, original not available), consists of graspins a stick and performing trembling head movements while moving the head towards the feet. A stick nay be grasped at its middle or at an end, and if it does not enter the framework easily it is withdrawn and the movement repeated again and again. The only other type of movement is named here "push-pull"; to remove a branch or reed close to the nest the bird grasps it in its beak and violently jerks it back-and-forth.

The first few sticks of the nest structure are placed haphazardly in the fork of a tree. Many of them slip out of place but are grabbed and placed again. Large numbers (estimated $30 \%$ ) of sticks accidentally fall after having been mani- 
Table 7. Iist of actions and probable releasers used by A. ibis in nest-building

\begin{tabular}{|l|l|l|}
\hline releaser & male action & \multicolumn{1}{c|}{ female action } \\
\hline $\begin{array}{l}\text { nest-site } \\
\text { criss-cross } \\
\text { platform }\end{array}$ & $\begin{array}{l}\text { collecting } \\
\text { sticks }\end{array}$ & $\begin{array}{l}\text { tremble-shoving } \\
\text { sticks, mostly on } \\
\text { a hoŕizontal plane }\end{array}$ \\
\hline saucer-shaped & $\begin{array}{l}\text { sticks } \\
\text { nest }\end{array}$ & $\begin{array}{l}\text { tremble-shoving } \\
\text { sticks at angles } \\
\text { other than horizon- } \\
\text { tal }\end{array}$ \\
\hline & sticks & $\begin{array}{l}\text { tremble-shoving } \\
\text { sticks at angles } \\
\text { other than horizon- } \\
\text { tal }\end{array}$ \\
\hline
\end{tabular}

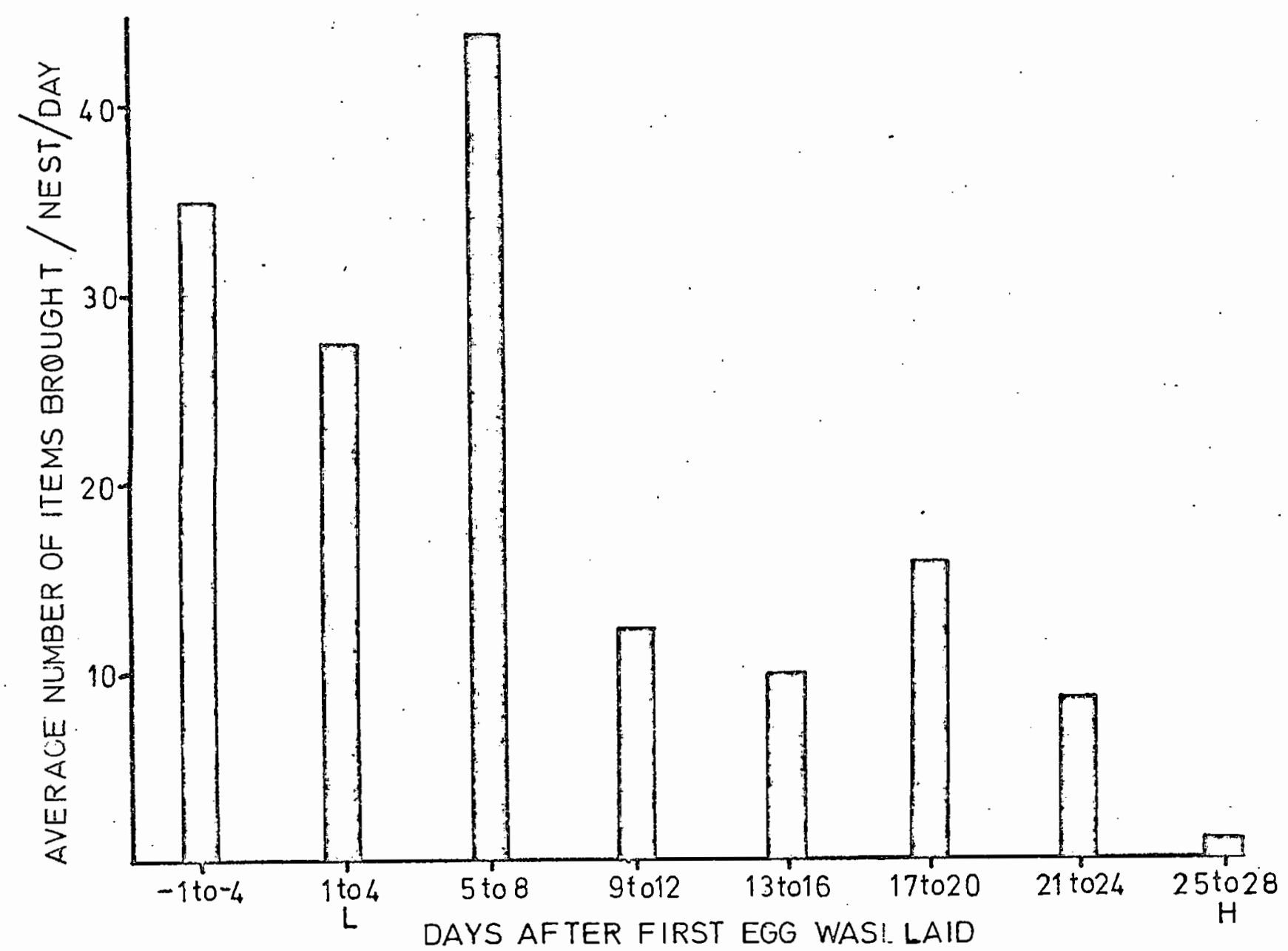

FIGURE 29. Average number of nest-material items brought per day. (one day $=0630$ - 2830 hours, all fizgre averages for 8 nests, observations standardised 0630 - 1230, 1230 - 1830 on alternate days.) $I=$ laying. $H=$ hatching. 
pulated for long periods, but the birds never discard a stick, even if it is impossibly big to be fitted into the structure. Instead, a sort of natural selection of material exists whereby big and awkwardly shaped sticks eventually fall. After about two days of this apparently haphazard trembleshoving of nest material, about 20-40 sticks have accumulated. A nest's shape at its different stages is to a large extent affected by the branches which support it, but a pattern is evident. The first few sticks are in the form of a loose crisscross platform (or bundle if in a fork). Once this horizontal platform is established, many sticks are inserted at angles other than the horizontal, and a shallow bowl is formed. This continues throughout incubation, and even when no new sticks. are available, sitting birds (both sexes) frequently trembleshove parts of the nest framework and loose sticks are either tremble-shoved further or withdrawn and re-inserted.

Compared with the number of complex actions (14) and releasers (18) which linbergen (19539, in Thorpe 1956) lists in the tit Aegithalos caudatus, nest-building in Ardeidae is a very simple process. Table 7, drawn up along the same line as Tinbergen's, shows that there are three basic releasers and two busic actions in nest-building by Ardeola ibis females. The range of nest material is also very narrow. Although no measurements are available, the author obtained the impression that the average length of sticks collected decreased as incubation proceeded

A number of points suggest that nest-building techniques of A. ibis (and presumably other Ardeidae as well) are better suited to reedbed- than to tree-nesting. Firstiy, most nests are extremely flimsy at the time of egg-laying, and figure 29 indicates that at this stage the nest is only roughly one third of its final size. This must be a major factor in egg-loss, for the bifgest single cause of egg-loss is gales which cause the trees to sway violently and eggs to fall, particularly from incomplete nests. This cannot, of course, happen in A. ibis nests built almost at water level in a marsh. "Secondly, for the same reason as above the nests' shallowness appears to be maladaptive in a tree-nesting bird. Finally, the trembleshoving actions prevent any form of nest-weaving taking place. A platform-type nest is presumably less secure in a tree than is a woven nest. Many Ardeidae nest in marshes and reedbeds but others, A. ibis included, prefer trees. The above three 
points suggest that nest-building technique has not become adapted to tree-nesting in those species which have abandoned the ancestral habitat.

\section{5,53 Social aspects}

Since $96 \%$ of nest-material is collected by the male, most nest-buildine takes place when the female returns after a period of absence, consequently most stick-collecting activity coincides with the morning and afternoon changeover peaks. Arrival of the male with a stick is always accompanied by a greeting ceremony. Stick-collecting by the male may last an hour or more and the maximum observed number of sticks brought after a single changeover was 35. During the first few days of the pair relationship the male often attempts to tremble-shove the sticks he has collected, but the female always pulls the stick away and places it herself; later the male always stanas silently on the rim of the nest and waits for the ferrale to take the stick. If she does not, he just puts it down and walks off. Before pairing, males frequently snap off twigs from their perches and tremble-shove them, but no nest frameworks develop.

The majority of nest material is collected at favoured sites 50 to 400 metres away from the colony, and up to 100 birds may be present at any one time at one of these sites. At Paarl in 1966 there were 6 sites, one of them a dead twiggy tree and the others patches of dead weeds, whose positions are given in Figure 24. Gatherings of bixds at these sites are clearly gregarious, and not just aggregations at areas providing suitable nest material; for all birds often switch from one site to another and back several times. When nest-building is at a peak there is a continuous stream of birds between the colony and one or two of these sites. Individuals will pick up and drop up to 10 sticks before selecting one. There is no displaying at these sites other than occasional lowintensity Forward Displays and Rick-racks.

The second most important source of nest material is the large numbers of sticks which fall out of nests and lie on the ground below the colony. Lastly, A. ibis readily steal sticks from unguarded nests, and nests abandoned in september and october are usually demolished in two days. A stickstealing bird will usually eject eggs and chicks from an unguaraed nest. Incubating birds (both sexes) occasionally 
reach out and tug at twigs on a neighbour's nest.

\section{$\underline{5.6 \quad \text { Incubation }}$}

Out of 155 marked nests which were visited over periods of at least five days, there was a maximum of two eggs in $31.0 \%$, three in $60.0 \%$, four in $7.7 \%$ and five in $1.3 \%$; average clutch size 2.86 . (One-egg nests were ignored as they had all presumably lost eggs). The average clutch size is probably about 10\% too low, since egg-loss occurred throughout incubation (figures below), particularly during egg-laying. The interval between laying of successive eggs was two days in 13 cases, three days in two cases and one day in one case. With one exception all eggs were laid outside observation periods; this egg was laid at 0650 so presumably the majority of eggs are laid in the early morning, as is the case in most birds. Incubation periods for 20 individual eggs varied from 22 to 26 days; mean 23.7 days. The interval between hatching of consecutive eggs was one day in 12 cases and two days in one case. Since eggs are mostly laid at two-day intervals and incubation begins immediately after laying, this implies that incubation periods for third eggs are shorter than for second, and second eggs shorter than for first eggs.

Egg-survival was calculated by a modification of Mayfield's (1961) method: 61 nests were observed for a total of 3078 egg-days and 25 eggs disappeared; probability of survival of one egg for one day is therefore 0.991877 , so the chances $P$ of one egg surviving for 24 days $=(0.991877)^{24}=$ 0.8241 , i.e. $17.6 \%$ egg loss. The chief cause of egg-loss was strong winds.which blew eggs out of the nests, particularly nests in the earlier stages of construction. Human interference was a major factor in egg-loss in this respect, for as long as an adult is sitting there is little chance of an egg falling out, even from a violently swaying nest. The only other figures on egg-loss in Ardeidae are those of Teal (1965), who found that $44 \%$ of 99 Casmerodius alba eggs, 33\% of 96 Egretta thula eggs, $19 \%$ of 47 Hydranassa tricolor eggs and $0 \%$ of 24 Nycticorax nycticorax eggs were destroyed by predators. All except one of these figures are higher than the $17.6 \%$ found for A. ibis and the heavy predation is particularly striking (Teal mentions no other cause of egg loss), which supports earlier conclusions that 
A. ibis are relatively immune to predation. In the present study, predation on eggs (by Nycticorax nycticorax) was actually observed only once, and probably accounts for a minor fraction of the $17.6 \%$.

\section{$17.2 \%$ of 99 eggs which were checked daily over the} period when chicks were due to emerge failed to hatch. This represents an extremely high wastage of reproductive effort: Knabe (in Lowe 1954) found a corresponding percentage of 3 in Ardea ... cinerea and the figures for Teal's (1965) 4 species varied from 2 to $8 \%$. There was no obvious cause for the high percentage in Ardeola ibis, although the inexplicably low copulation rate may be a factor.

Clutch-size in A. ibis is determinate. In four nests the second and third eggs were removed within six hours of laying; in three cases a total of three eggs were laid at normal intervals and once two eggs were laid, so it may be assumed that normal clutches were laid in each case and that egs-laying was not stimulated.

Figures on the daily cycles of attentiveness are given in Section 5.8. There is very little activity during the incubation period and apart from the noise of greeting ceremonies during the morning and evening changeovers, the chief activity is occasional Forward Displays directed at a neighbour. which is moving around on its nest. Birds seldom sit inactively for long periods, but frequently stand, turn around and tremble-shove part of the nest framework before sitting again. Incubating birds sometimes sleep with their heads resting on the nest rim.

Eggshells are dropped over the side of the nest soon after hatching. Tinbergen/(1962) showed that eggshell-removal in Iarus ridibundus is an anti-predator device which renders the nest less conspicuous, so absence of any special eggsheliremoval pattern in $\mathbb{A}$. ibis is additional evidence that the nesting birds are little affected by predation.

\section{$\underline{5.7 \text { Nestling period }}$}

The interval between hatching of consecutive-laid egrg was one day in 12 cases and two days in one case. Asynchronous hatching of the eggs is an extremely important factor in the biology of young birds; details are given in 
Section 6.22. For reasons stated in Section 6.16, it is difficult to assess accurately the duration of the nestling period, but the chicks cease to be fed when they are 35-55 days old; it is not known whether this is initiated by departure of the chicks or break-up of the pair. Figures on feeding rates and chick mortality and descriptions of causes of mortality, chick behaviour, feeding methods and parent-young recognition are all presented in Section 6, where they have greater relevance.

Hatching does not apparently affect the pair relationship at first and normal greeting ceremonies continue to be given at each changeover, but after brooding ceases the pair meet more and more infrequently. By comparison with incubation, the nestling period is one of great activity, with continuous noisy chick begging. In October-November there are almost always birds flying between the colony and feeding areas, with birds usually returning to the colony in small groups which split up as the individuals go to their respective nests.

\subsection{Attentiveness}

\subsection{Pair-formation stage}

Details are given elsewhere of the behaviour of adults at this stage, the frequency with which pairs change nest sites, and the duration of the pair formation stage. During this period, the nest site is occupied by at least one member of the pair for an average $96 \%$ of the time (sample 86 hours, standardised as described in section 1.2). Over the same sample period, males spent a total of $94 \%$ of their time on the nest site, females $38 \%$ and the pair together $35 \%$. Males spend very little time away from their nests at this stage, presumably relying on the large fat reserves which they possess during the early breeding season. (Siegfried pers. comm.). For two or three days before the eggs are laid the empty nest is sat on for an increasing percentage of the time.

\subsection{Incubation}

As in all Ardeidae (Kendeigh 1952), full-time incubation begins with laying of the first egg. Details on the spacing of egg-laying are given in section 5.6. From the moment of egglaying until the young are at least 10 days $01 d$, the nest is 
never left unguarded. In the terminology of Skutch (1962) the constancy of incubation is $100 \%$, although an incubating male may very occasionally leave the nest for a minute to fetch nesting material. It is not known whether this high percentage of attentiveness occurs in other Ardeidae, but its function in A. ibis is almost certainly to protect the eggs and young from predators and the nest itself from stick-stealing birds. Predation by Nycticorax nycticorax (details in section 2.4) usually occurs when the incubating A. ibis has been forced to leave the nest by the presence of a human, implying that under natural conditions A. ibis nests are protected from most predators. As regards stick-stealing (described in section 5.5), a nest abandoned by its owners may be demolished within two days when nest building is at its peak.

Attentive periods in A. ibis vary in duration from 3 to 26 hours and there may be one, two or three per day, but within these limits there is a strongly marked diurnal rhythm of attentiveness. (Kendeigh (1952), in his review of the Ardeidae, stated that attentive periods last 2 to 6 hours but this may be based on incomplete observation). In some pairs, A. ibis' attentive patterns change as incubation proceeds, so the first and second halves of incubation have been dealt with separately.

Figure 30 shows that during the first half of incubation there are peak frequencies of changeover around 0900 and 1600 hours. This indicates that one bird normally sits from about 0900 to 1600 and its mate from 1600 to 0900 the next morning. There is no suggestion that either sex tends to be confined to either period, for during all-night observations females were sitting in 18 cases and males in 14 cases. It is obvious that if one member of a pair is not to be restricted to the daytime period and the other to the night period, there must occasionally be an extra changeover, and there were in fact three changeovers on $25 \%$ of the days in the first half of incubation. The times of changeover for individual pairs from one day to the next are remarkably constant, sometimes varying by as little as 5 minutes on consecutive days. Incidentally, this regular 24-hour cycle appears, fully developed, approximately at the same time as the last trace of orange fades from the birds' beaks, usually two or three days before egg laying. Prior to this, no pattern. can be detected in paired birds' attendance and non-attendance at the nest. 


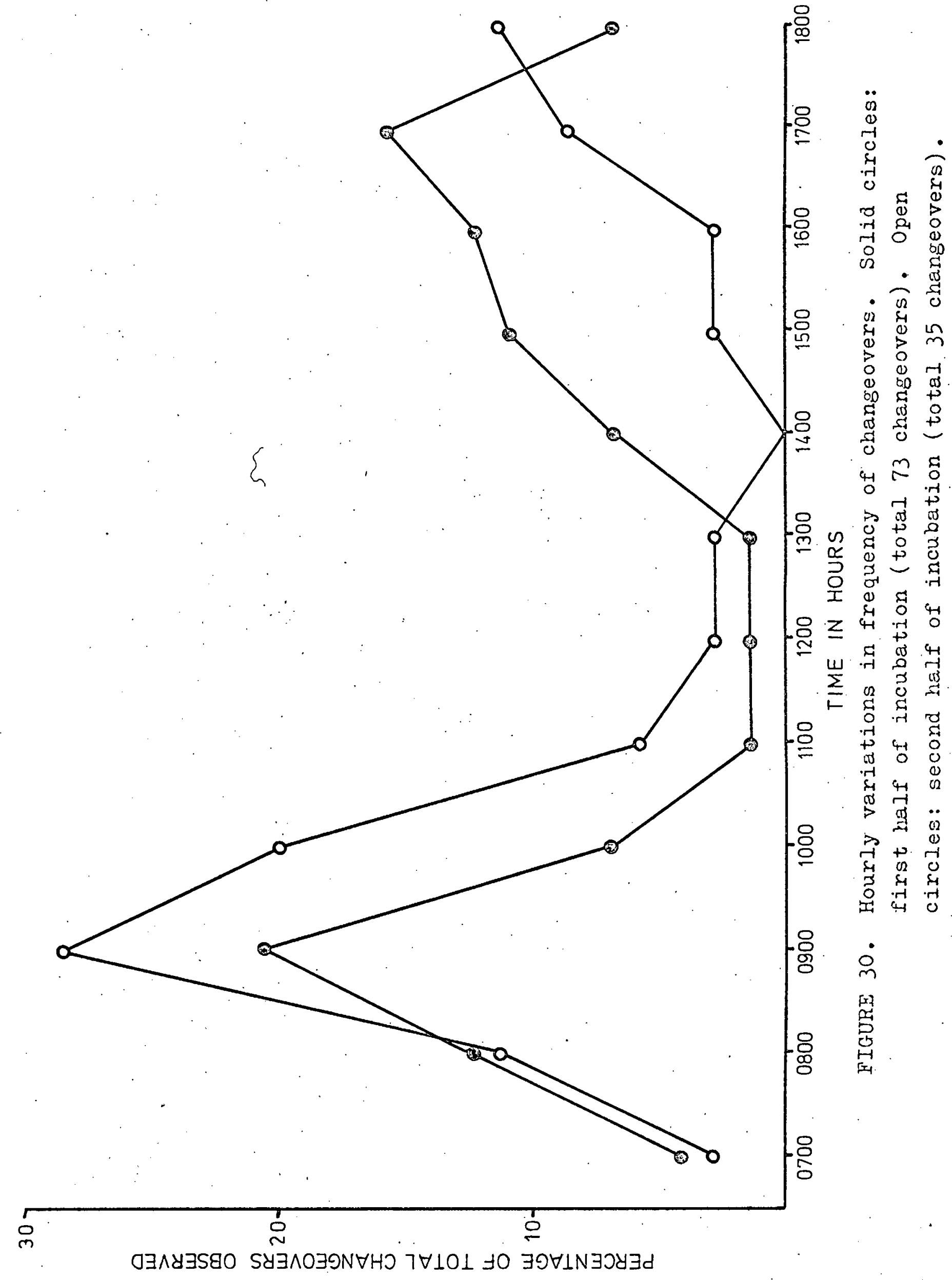


Figure 30 indicates a different daily cycle during the second half of the incubation period, for the majority (69\%) of changeovers occurred during the mornings. This was due to the fact that three out of the eight pairs only changed over once per day at this stage and followed a 48-hour cycle. Two of the pairs retained the 24-hour cycle and three alternated between 24 and 48-hour cycles. During the 48-hour cycles, each member of the pair sits for approximately 24 hours. During one all-night observation of 8 nests, only one bird was present at each nest, the "off" birds presumably sleeping in the treetop above the nest. On two occasions small groups of birds have been seen leaving nesting colonies after sunset, but otherwise no nocturnal activities of any sort were noticed. No reason can be seen for the long attentive and inattentive periods. In many groups (e.g. Procellariformes), lengthy attentive periods make it possible for the birds to forage over a wide area, but this effect cannot operate in A. ibis which rarely feed more than an hour's flying time away from the nest. The actual percentages of daytime (by hours) spent on the nest by the female A. ibis are not presented in table form, as recommended by Kendeigh (1952) and Moreau and Pitelka (2943), because the birds have 24-hour attentive cycles, and the system of alternate morning and afternoon observation periods could have caused artefacts. It is likely that a sufficiently large sample would show that incubation is shared exactiy 50-50.

Although the nests are never unattended, the birds do not of course sit without a break. Much time is spend standing, preering, arranging nest material and turning eggs. Close observations of nests for a total of 22 hours over a range of conditions gave an average of $96 \%$ of the time spent sitting when the temperature was below $20^{\circ} \mathrm{C}$ ( 14 hours) and 71 when the temperature was above $20^{\circ} \mathrm{C}$ ( 8 hours). Wind also has a strong inhibiting effect on the tendency of incubating birds to stand. Baerends (1959) deals with the effect of external factors on incubation behaviour.

\subsection{Nestling period; Feeding rates.}

The actual process of hatching, behaviour of the chicks, feeding methods, and responses of the adults towards their young are described in section 6. Chicks are brooded almost contin-: uously for the first 4 to 9 days of life, and thereafter the adult alternately broods the chicks, or stands "on guard" nearby. 


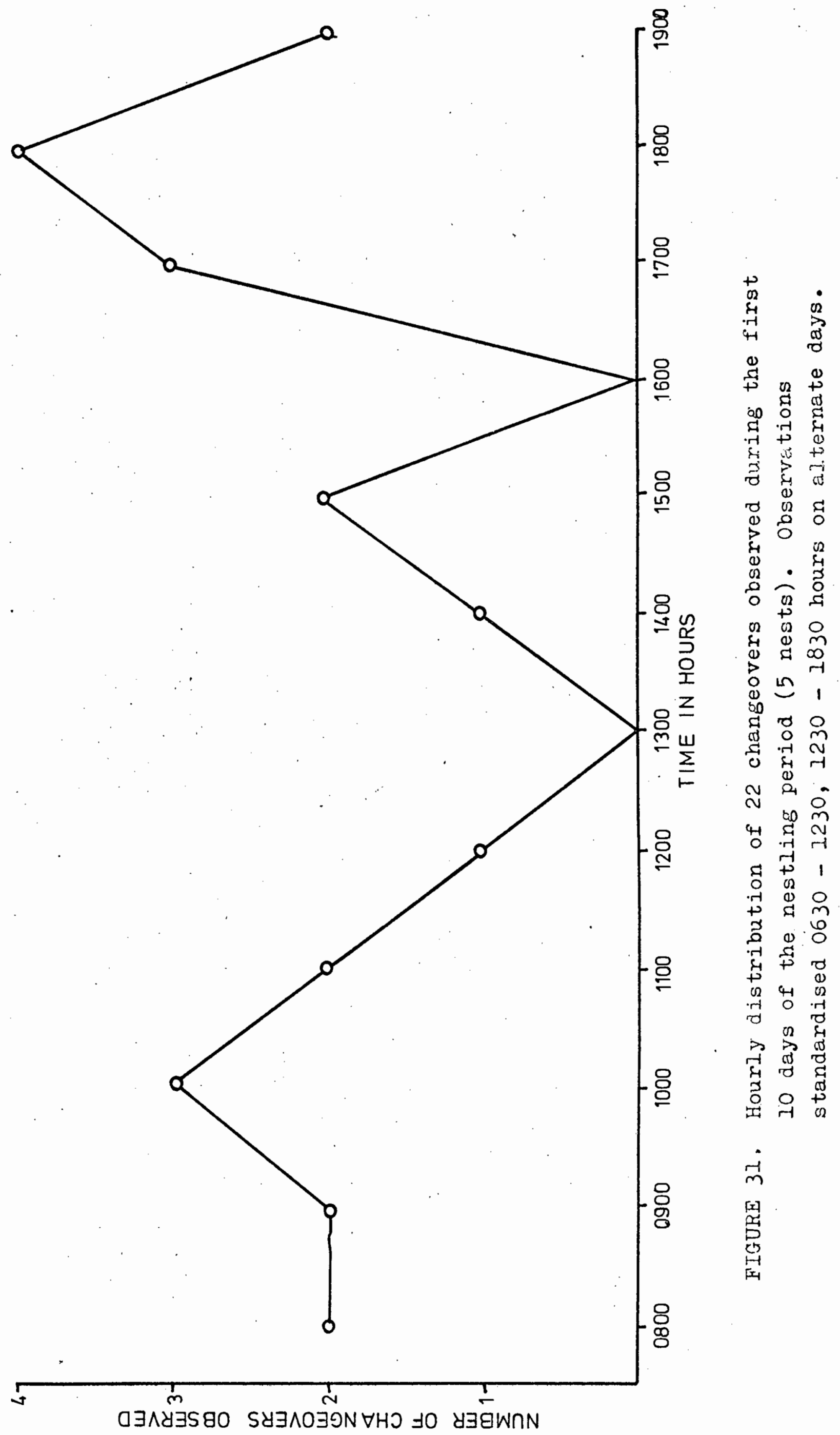


$\stackrel{\infty}{\infty}$

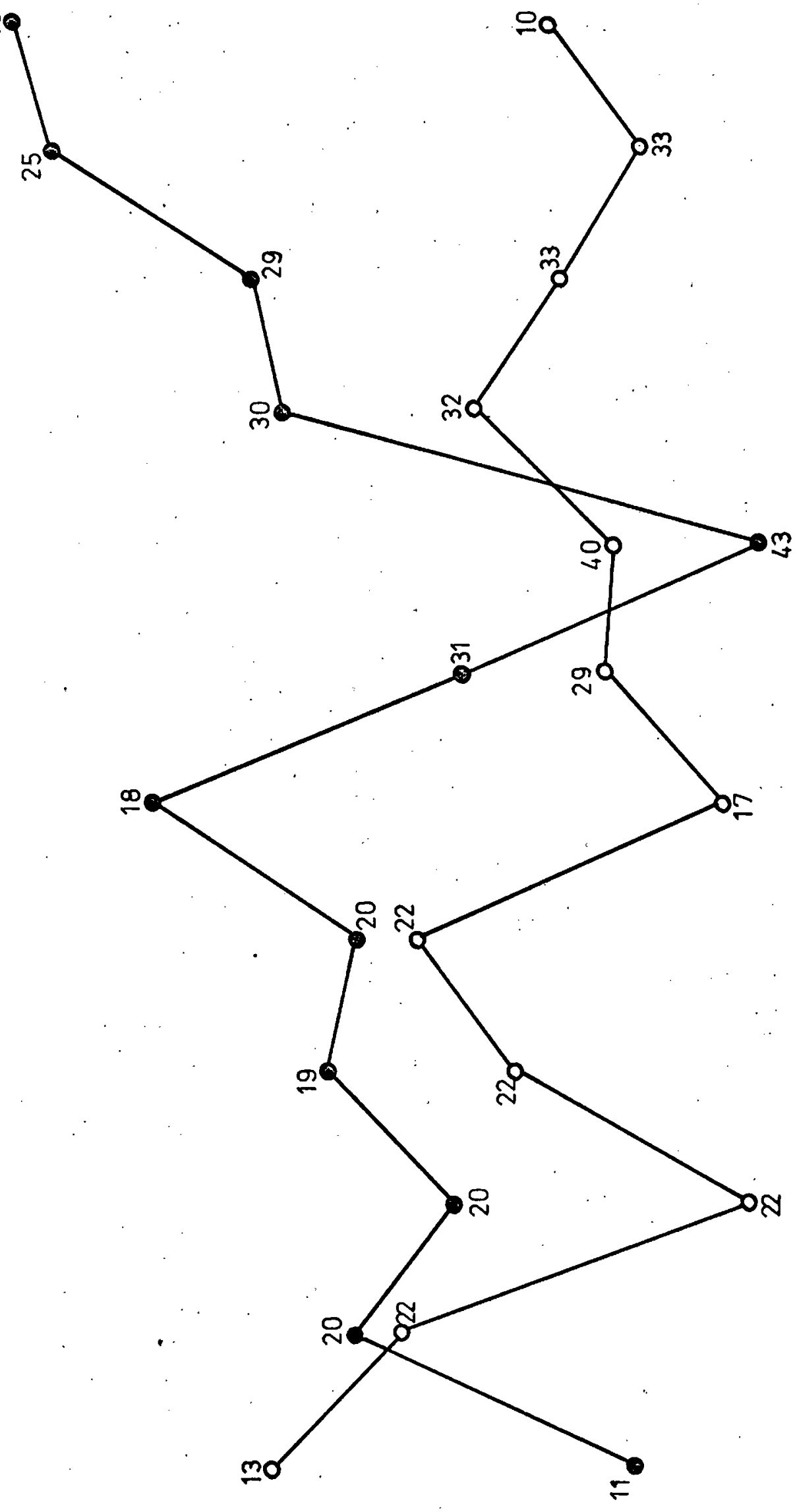

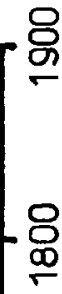

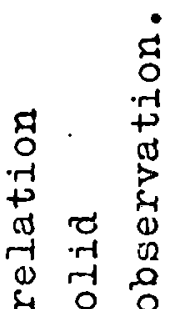

응

H

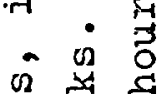

का

둉

त्र 0 \&

द 它

द्व

- $\quad$ \&

$\begin{array}{ccc}4 & & \\ 0 & 0 & 0 \\ -1 & 0 & 1 \\ 0 & 0 & 0 \\ 0 & 0 & 0\end{array}$

$\begin{array}{llll}8 & 0 & r & 0 \\ 8 & 0 & 0 & 0 \\ 0 & 0 & 0 & 0\end{array}$

음

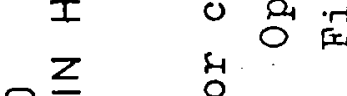

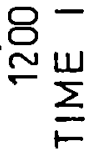

क 1.

a) $\overrightarrow{0}$

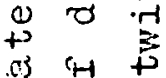

त)

-8 $\quad$ 일

तु भु

D) 0 \&

\$

$\dot{\sim}$

$m$

䍃

每

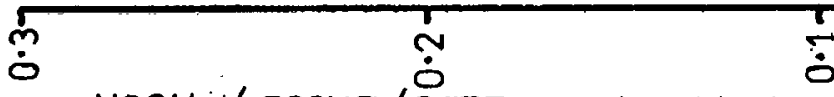

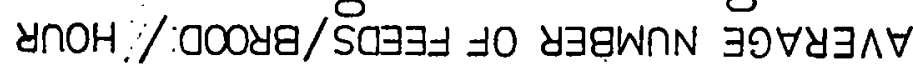




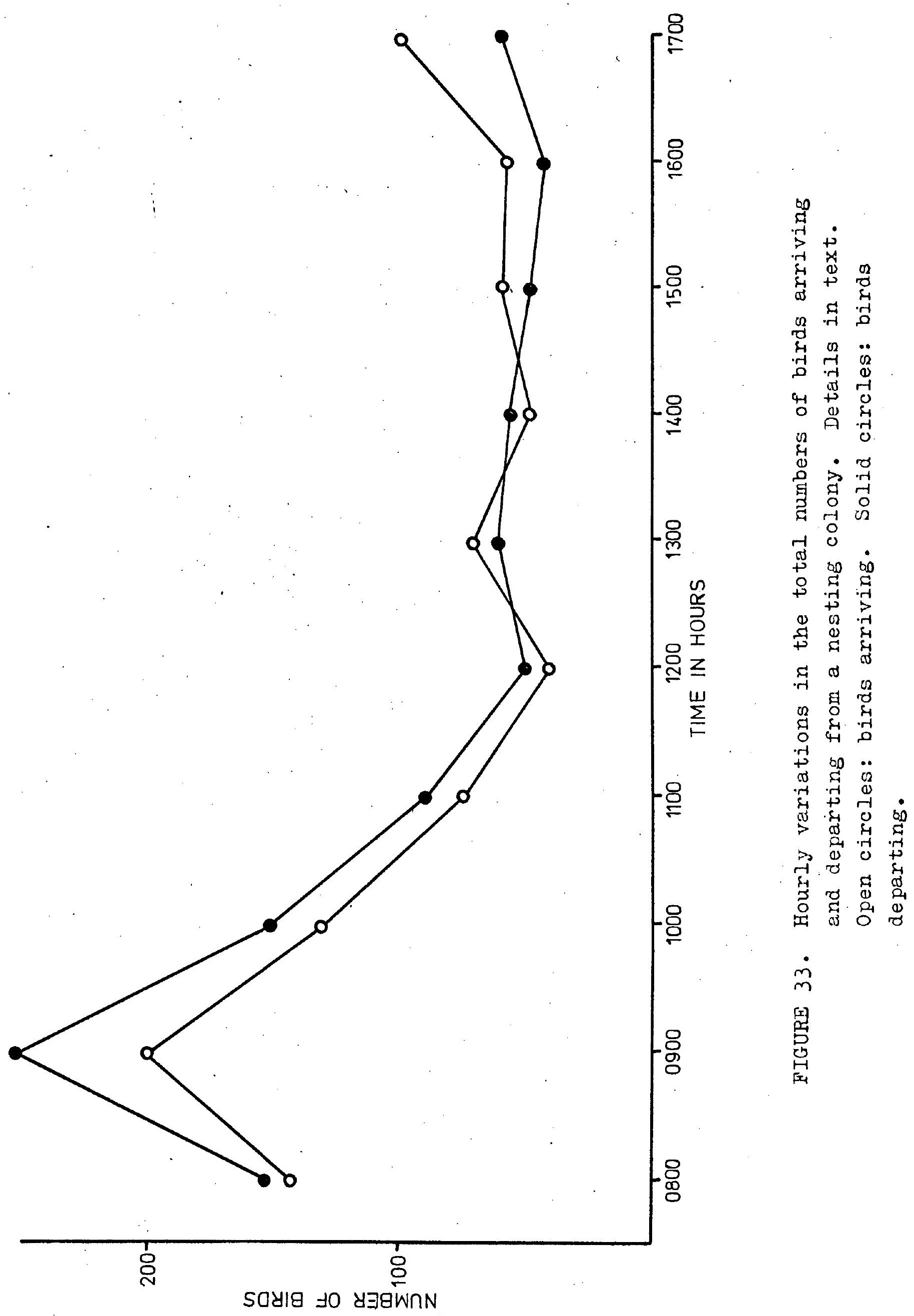


Guarding ceases finally 12 to 19 days after hatching of the first egg, but there is some variation in this respect. Guarding ceased finally at 12,12 and 15 days in the three nests of twins, and at 15, 16 and 19 days in three single-chick nests. This effect is possibly due to the fact that parents of twins have to spend inore time finding food than parents of singletons do. This must be an important factor promoting survival of single chicks, as cospared to twins, for when guarding ceases they are too large to be eaten by Nycticorax nycticorax.

Hatching of the chicks does not initially have much effect on the parents' attentive rhythm. Figure 31, which gives the distribution by hours of 22 changeovers observed during the first 10 days of the nestling period, shows that there are definite peaks at 1000 and 1800 hours, suggesting a continuation of the diurnal rhythm which exists during incubation, (Four nests, observation standardised as described in section 2).

A "feed" is defined here as a period lasting up to 20 minutes during which at least one bolus of food is passed from the parent to its chick(s) (detailed description in section 6.21). Immediately after 19 of the 22 changeovers observed at the 1-10 day stage, the recently-arrived parent fed its chicks. Until the following changeover the chicks were usually fed one to three more times, but detailed figures are not presented here because many feeds, being inconspicuous, may have been missed. The important point to notice is that each parent only makes one collecting trip for food each day.

Figure 32 gives the feeding rates, by hours, for one-andtwo-chick broods older than 10 days. (It was necessary to take into account the total amount of observation for each hour, for observations at this stage did not all follow the 0630 - 1230 , 1230 - 1830 hours standardisation). There is little indication of any pattern in the hourly feeding rates, other than a possible early morning peak for single chicks and an evening peak for twins, but it is likely that these peaks are artefacts caused by inadequate samples. Figure 33 gives the hourly totals of arriving and departing adults at another colony, at a stage when all chicks were older than about two weeks. It indicates a well-marked morning peak and suggests an evening peak of feeding, but some of the birds recorded were non- and post-breeders:

From the loth day on, the overall average feeding rates for single chicks was 1.5067 feeds per day; and for twins 2.4642 
feeds per day (total sample in each case 284 nest-hoursobservation on 3 nests). These figures were obtained by totalling the average feeding rates in figure 32 for each hour from 0800 to 1900. Feeding continued at a reduced rate between the end of the detailed observation periods (1930 hours) and sunset, so the above values are probably $5-20 \%$ lower than the true figures. Since the parents share ali attentive activities (also in Kendeigh 1952), each parent feeds its offspring an average of about 0.8 to 1 times per day in the case of single-chick broods, and 1.3 to 1.5 times a day in the case of twins. Because thirdhatched chicks rarely survived beyond 18 days, figures on triplets were too scanty.

The above figures are surprisingly low considering that adults collect their offspring's food well within an hour's flight of the nest. Owen (1955) found that young Ardea cinerea are "fed about $3-4$ times in 24 hours when one parent is feeding and about twice as often when both are feeding" (based on only 20 observed feeds). Kendeigh (1952) states that Ardeid chicks are fed 6 to 10 times per day. The long gaps between feeds in Ardeola ibis cannot be a mechanism which enables parents to spend a maximum percentage of their time collecting food, for parents of single chicks do not make as many food-obtaining trips as do parents of twins, although they must be capable of doing so. In addition, parents of single chicks sometimes, (but parents of twins, never) appear at the nest site and perch nearby for a few minutes without feeding the chick, suggesting that they are not fully occupied and could feed their offspring more frequently.

The second point to note is that the feeding rate for twins is less than double that for singletons (164\%). This could be partly due to the fact that the rate of heat loss per chick is less for larger broods, and their food requirements thus lower (Royama 1966), and partly because parents of twins may be approaching the limit of their food-obtaining ability.

\subsection{Interactions outside the pair}

The complexity of a species' social organisation is indicated by the type of interactions which occur between individuals which are not mated. A. ibis' social life is very simple, and this study's programme of individually marking nesting birds was singularly unproductive. There is no evidence of individual recognition (in adults) outside of the pair and all interaction 
among unmated birds can be recognised as overt fear, aggression or sexual behaviour. Even when breeding, A. ibis fits exactly into category 2 (i) in Crook's (1965) system of classification: "Flocks without constant composition in which no inter-individual relationships based on personal recognition occur". "Although the complex behaviour sequences which occur during the mateselection stage are part of extra-pair relationships they are dealt with in section 6.21 .

\subsection{Aggression}

Agonistic encounters are the commonest extra-pair interactions. They usually take the form of a rapid exchange of several Forward Displays between birds occupying adjacent nests. The form of these encounters and their ritualised back-and-forth stabbing movements in which neither bira touches the other, have been dealt with in section 3.21. These encounters are usually caused by a disturbance such as a greeting ceremony at one of the nests. Trespassing birds are never tolerated closer than about $80 \mathrm{~cm}$. from the centre of the nest, although the exact distance varies and is partly dependent on the behaviour of the intruder. On the approach of a trespasser, a territory owner will slowly stand, raise all plumes and perform a Forward Display if the other bird comes closer. Figure 8 gives the frequency of extra-pair agonistic encounters, most of which are between birds owning adjacent nests.

It is evident from the description of the stab-exchanges between birds on neighbouring nests (section 3.11) that the minimum distance between nests is not determined by the distance which a sitting bird can peck. The maximum size of the defended area was very poorly defined but normally extended for two or three metres radius around the nest. In one exceptional case a nest-owner supplanted a bird 7 metres distant.

No figures could be produced on the relative aggressiveness between neighbours and between individuals which did not occupy adjacent nests. However, I did not obtain the impression that nest-owners were any less aggressive towards neighbours than they were towards "strangers". Lorenz (1966) comments similarly on Nycticorax nycticorax. This is probably a very significant point because one would expect the first basis of organisation within a society to be elimination of unnecessary territorial aggression between neighbours. Figures on differences between 
A. ibis, Egretta garzetta and E. intermedia as regards intraspecific aggression could not be obtained because nest-densities for each of the three species varied greatly. However, I obtained the strong impression that $A$. ibis are relatively much less agsressive towards couspecifics than the Egretta species are; fighting is particularly common in E. Earzetta.

\subsection{Copulation between non-paired birds.}

Copulation outside the pair is infrequent and does not play a.major part in the species' biology. Seven attempted and four successful extra-pair matings have been seen, two of the latter involving the same female. All 1 incidents occurred during the first week of the females' incubation period, and all while the female concerned was on the nest and her mate absent. On each occasion, a male left its nest nearby, flew straight onto the sitting female's back, and attempted to copulate in the nornal manner. In four cases the female was fully receptive, crouched as in normal copulation, and paid no attention to the male when it flew back to its own nest immediately after dismounting. Both birds continued their previous activities as if nothing had intervened. In the remaining cases the female attacked the male as it approached the nest but the male persisted in trying to mount before being repelled with wild,wingflapping by both biris. This usually generated much excitement among several males nearby, with two or three flying to the female and a pecking fight with much flapping occurring between all the birds on the nest before the males were driven off by the resident female. These attempted "rapes" are never successful. Meanley (1955) observed more extra- than intra-pair copulations in Florida caerulea.

It is not easy to see what factors influence the female's receptivity or hostility. In 30 minutes one male attempting three copulations with a female sitting on a nest 5 metres away was rejected, accepted, then rejected again, so individual preferences of the female must be of little importance. One final point of interest concerns the behaviour of the mates of the birds involved. In neither of the two occasions was a female seen to pay any attention when its mate copulated with another female nearby. This strongly suggests that the pair bond exists only while the birds are" on the nest site. Lorenz (1966) obteined the same impression for Nycticorax nycticorax. 


\section{BEHAVIOUR OF YOUNG A. IBIS}

\subsection{Maintenance Activities}

\subsection{Hetching}

Figures on clutch size, the percentage of eggs which fail to hatch, and the intervals between successive hatchings are given in section 5.6. For 12 hours or more before a chick emerges it gives weak, drawn-out notes, rendered "eeeh", which after hatching are discomfort notes, given when the chick is cold or roughly handled. (Vince (1964) has shown that pre-natal calls of quail embryos stimulate embryos in other eggs and accelerate their hatching.) On emergence from its shell, a chick's first responses are weak kicking and grasping movements of the feet, which serve to right it, and during the first hour of life its only other behaviour is jaw-stretch, "eeeh" calls and shuffling locomotory movements. Parents have not been observed to assist the chick from its shell. As in all other Ardeidae (Nice 1962), A. ibis chicks are hatched with their eyes open and are covered in down, which dries and becomes fluffy after a few hours.

\subsection{Comfort activities}

All adult comfort movements are present by the time chicks are 22 to 25 days old. Jaw-stretch sometimes appears within an hour of hatching and later becomes very common, particularly on awakening. Body-shake has first been observed at three days, but in a poorly co-ordinated from, and the rotary headshake component is frequently given without body-shake. Perfunctory nibbling-preening has first been seen on the fourth day, chicks becoming expert by 20 days. Underwing preening has been seen at 18 days; both-wing stretch and one-leg-and-onewing stretch have first been observed at 9 and 20 days respectiveIy. Normal adult scratching movements have been observed at 22 days. Chicks younger than about a month usually take on or two backward steps before defaecating, but the movement is not directed for chicks will shuffle backwards even if placed on a featureless surface.

\subsection{Behavioural Thermoregulation}

The commonest response to high air temperature and solar radiation is gular flutter, which is present from the first day and becomes less frequent as feathering proceeds and insulation 
improves. When gular-fluttering, young birds never clump together. During hot weather, chicks often lie limp on the nest with neck outstretched, a position which probably provides the maximum surface/volume ratio. Although nestlings sometimes perch in the shade it is not certain whether they actively seek it, and no special shade-providing position of parent birds has been noticed. No chicks were observed to die of overheating, although shade temperatures at Paarl sometimes exceeded $35^{\circ} \mathrm{C}$.

The commonest response to low environmental

temperatures is clumping, in which siblings squat on their tarsometatarsi in the centre of their nest, huddling together and facing inwards towards each other. Clumping occurs in unbrooded chicks of all ages. While singletons cannot clump, they squat in the same hunched position with neck retracted and beak against the breast feathers. Chicks shiver when their body temperatures are very low and particularly when they are wet. Sunbathing is infrequent; the bird stands with its back to the sun, wings drooped and slightly spread, all feathers ruffled (as in Meyerrieck's illustration); first seen at 15 days. In Ardeola ibis, deaths due to cold occasionally occurred but many of these may have been directly caused by the parents abandoning the nest.

\subsection{Climbing and the tendency to wander}

In common with all other young Ardeidae which the author has observed, young A. ibis are proficient climbers and can scramble around the nesting trees. A climbing chick often uses its beak as a hook as illustrated in Figure 34, and also bites hold of twigs, but the wings are not often used. The foot grip develops rapidly, a day-old chick being able to hold on with beak and feet for 5 to 30 seconds, while a 20-day chick can easily pull itself up with one foot. If a chick (any age) is picked up, it invariably performs rhythmic kicking and grasping leg-movements. This may be a reflex, for the movements are seldom directed at a perch, and if one foot grabs the other, kicking usually ceases.

This climbing ability of A. ibis chicks probably serves chiefly as a predator-response, for nestlings older than about 12 days react to humans by scrambling away through the cranches, which makes them very difficult to catch. Their climbing ability also helps misplaced chicks regain their nests, 


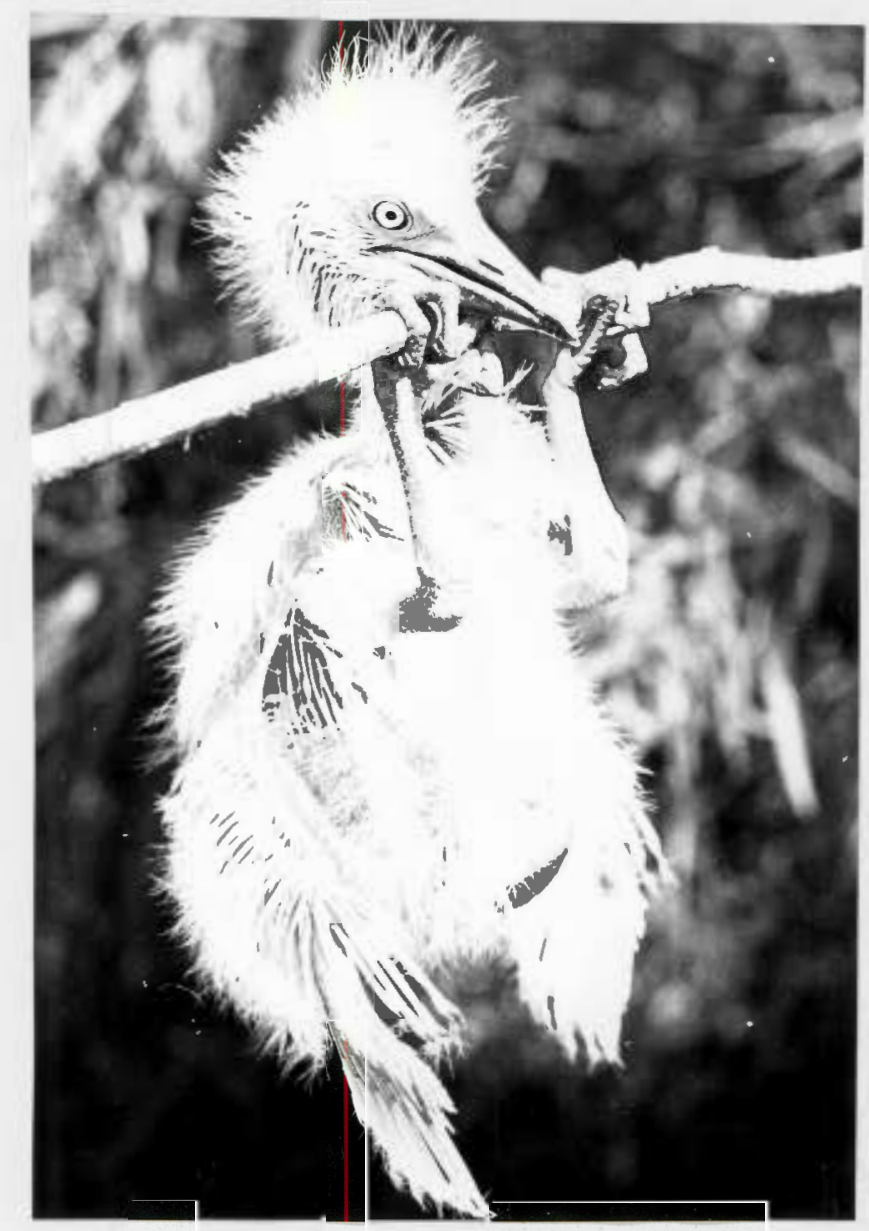

FIGURE 34. Chick (approx.il2 days) climbing; note use of beak. 
for barring attack bj a strange adult (below), a chick older than about 15 days. which happens to fall to a lower level in its tree can usually regain its nest. On several occasions nesttrees blew down in gales; some chicks died but many older ones climbed up again through the tangle of branches and continued to be fed by their parents.

As soon as they cease to be brooded, nestlings begin to wander around the nest-tree; the earliest a chick has been observed to leave its nest is at 9 days. Even when undisturbed, they walk around the branches sometimes crossing other nestterritories, and usually have a favourite perch two or three metres from the nest. Older chicks usually extend their defended territory (Section 6.31) to include a nearby abandoned nest if one is available. Nestlings older than about 30 days spend most of their time perched in the treetops above their nests, returning to the vicinity of the nest only to be fed." Siegfried (1966b) coins the appropriate name "branchers" for chicks older than 25 days. They are able to fly by this stage (details in section ó.16) and move around the colony, spending less and less time near their nests, and occasionally fly to the ground to feed. Chaiks older than about 40 days, and still being fed by their parents at their nests, often gather in flocks of up to 200 on the ground, resting and feeding. It is not known whether the prey they catch forms a significant percentage of their total intake. It is apparent from the above that a progressive weakening of site-attachment tendencies takes place. The immediate vicinity of the nest is defended until at least 30 days of age, while a chick's home range steadily increases in size, until a stage is reached where many chicks share common home ranges in the treetops, and seldom defend their nest-sites. The interactions resulting from trespassing are described in section 6.3 .

Nany deaths result directly and indirectly from persecution of trespassers. Quite often a chick perched at the end of a branch has its route back to its nest cut off by the return of an adult on a nearby nest. When the misplaced chick tries to regain its nest is is attacked and often either killed or knocked out of the tree. (Parent-young recognition is dealt.with in Section 6.33). Some older chicks which fall onto the ground are able to climb or fly up again, butydie of cold or starvation after a few days. The majority of chicks 


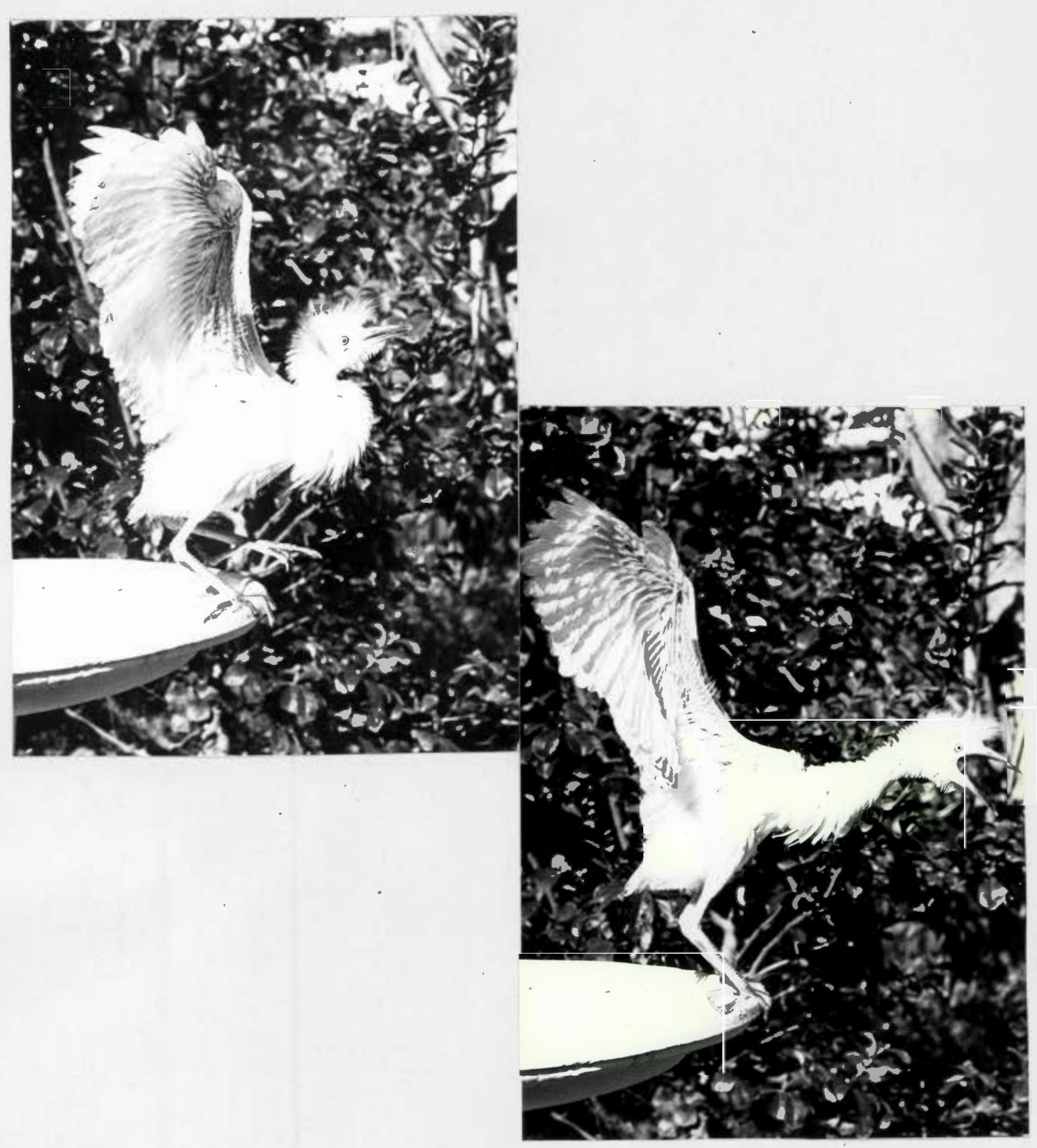

FIGURE 35. Chick (approx. 30 days) threatening a predator with Forward Display. 
which fall are already starving and weakened; this is expanded in Section 6.2.

Similar wandering and climbing of the young has been described in Florida caerulea (Meanley 1955), Butorides mufiventris (Uys and Clutton-Brock 1966) and B. striatus (Cowles 1930).

\subsection{Predator-responses}

With the exception of an incident with an eagle, described in section 2.4, the responses of wild nestlings to predators has not been observed; only their reactions to humans. Chicks younger than 8 to 12 days sometimes threaten humans and never show any sign of withdrawal or fear. Once this stage has been passed, chicks either crouch flat on their nest at the approach of a human, or scramble out. Predator-recognition is innate and not released by the alarm calls of adults, as it is in sore birds (e.g. Alley and Boyd 1950), for chicks removed from the colony retain their responses to cats and at first cintinue to be fearful of humans. At 15 days old, tame nestiings always responded very aggressively towards approaching cats and dogs, giving high intensity Forward Displays. The adult components of beak-stabbing, spread wings and harsh call are all present (Figure 35) and the open beak suddenly exposes the mcith's pink interior. This often has a startling effect on an unsuspecting human, so may function as aposematic display as regards predators.

\subsection{Flying}

From 20 days onwards nestlings frequently perform exercise wing-flapping (first seen at 14 days). The bird walks to the edge of its tree and vigorously flaps its wings for a few seconds while holding onto the perch. First flights, of ten oniy lour metres or so, are usually made at 25 to 30 days (earliest observation: 24 days) and all chicks can fly strongly by 30 to 35 days. Rapid, side-slipping swerving flight (Section 2.I) is frequently performed by young birds flying around the colony (also in Skead 1966). It is far more common in young than in adult birds, and may serve the purpose of giving young birds experience of a wide range of flight manoeuvres in a short period of time. 
Six colour-ringed chicks which were watched intensively were still on their nests at $38,46,54,55,55$ and 56 days, and giving a minimum average of 51 days. Most birds continued to be fed for at least 35 days, but it is difficult to determine accurately the date when birds finally leave the nest, for from about 30-35 days onwards they are seldom seen in the immediate vicinity of the nest. The lengthy interval between first flights and final departure from the nest ( 10 to 30 days in A. ibis) may be widespread in the Ardeidae for it has also been noticed in Egretta garzetta, E. intermedia and Nycticorax nycticorax (pers. obs.). This period is named the post-nestling stage by Skead (1966). During the post-nestling stage, A. ibis young fly up to 600 metres from their nests, land in other parts of the colony and feed on the ground, but always return to their nests to be fed by their parents.

Since at 25 to 30 days chicks are able to fly, feed themselves and perform all adult maintenance activities, the last 10-30 days of the period of dependence on their parents must represent a fledgeling period during which the chicks continue to be fed at the nest site. Birds in the colony are little affected by predation, so the system whereby the young spend the fledgeling period at the nest, as opposed to being fed by their parents away from the colony, must be an anti-predator adaptation. This is supported by the fact that in the cryptically coloured Ardeola ralloides, the joung are fed away from the mest (North 1963). The present state of affairs in A. ibis could easily have been arrived at by predator-selection against young which left the nest as soon as they were able to fly .

\subsection{Feeding and competition for food}

\subsection{Direct observation}

During the first 5 to 8 days of life, A. ibis chicks feed by weakly pecking and grabbing at any part of the adult's beak. When an adult is ready to pass food, it stands over the young in the hunched position illustrated in Figure 36, with beak pointing vertically downwards, and responds to its offsprings' pecking by slowly regurgitating a bolus of food. The chicks peck the bolus as it slides down the beak, but most food is eaten off the nest. Several ( 1 to 6 ) boluses are passed 


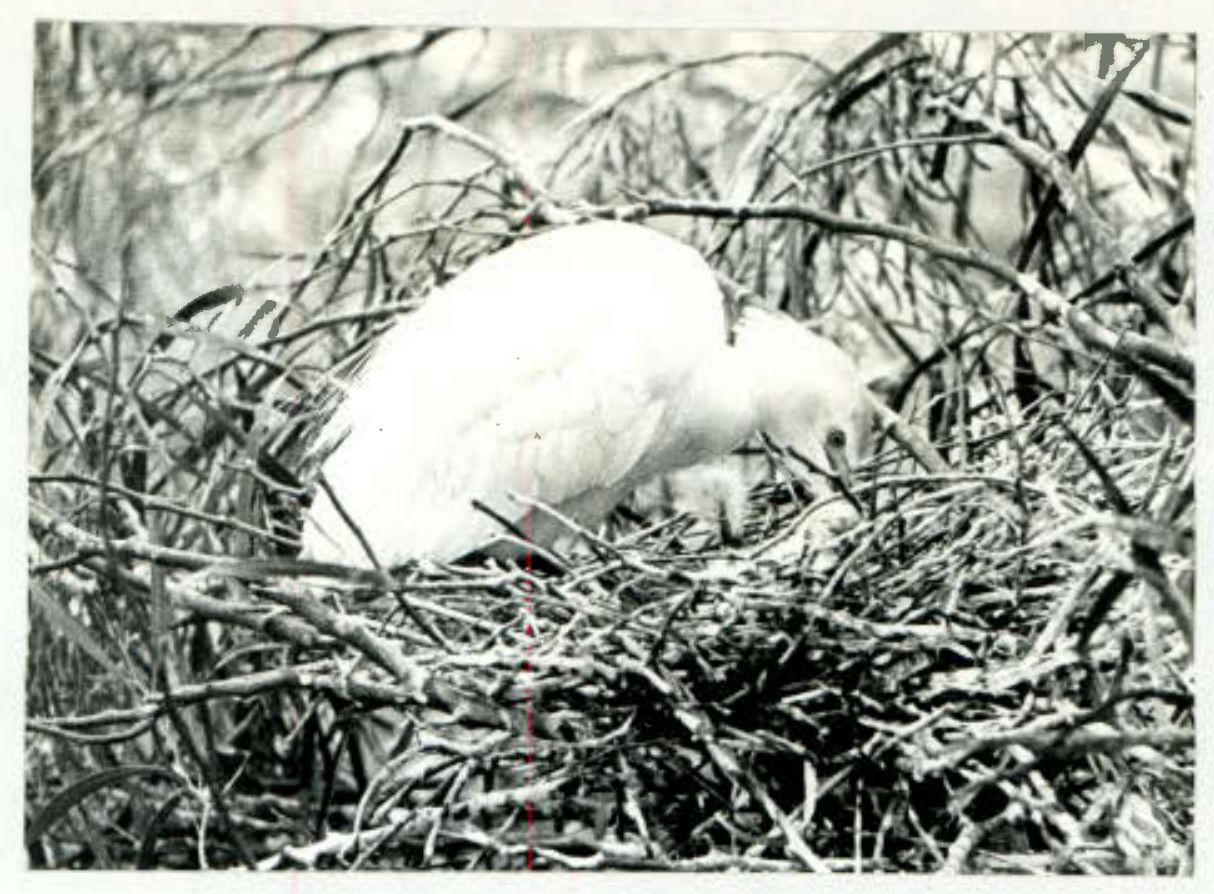

FIGURE 36. Hunched attitude of parent while feeding very young chicks.

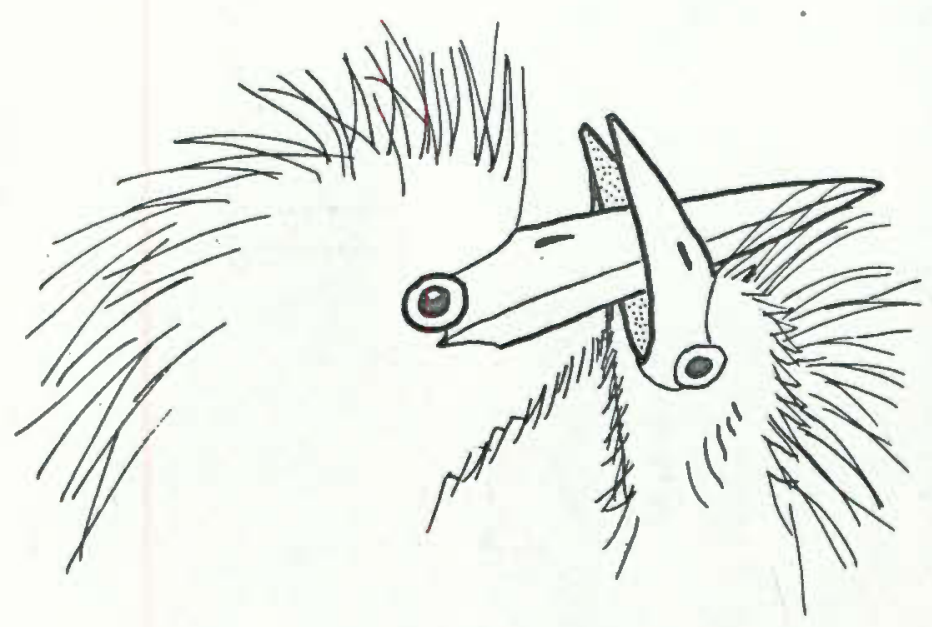

FIGUIIE 37. The grabbing feeding method of older chicks. 
during the course of a single feed, which lasts from 2 to 20 minutes. At this stage, a brood of chicks seldom eats all the food available, the adult re-consuming the uneaten remainder. Sometimes an adult regurgitates items such as entire frogs which are far too large for young chicks to swallow: these are always eaten again by the adult but may be re-regurgitated several times with the same results.

Begging in very young chicks consists mainly of a continuous two-syllabled note which Skead (1966) renders as "zit zit". Begging movements and calls are described in greater detail below.

As the chicks become older their grabs at the parent's beak become stronger until they are eventually able to grab the parent's beak firmly crosswise near the base (Figure 37), all food being passed directly from the parent's beak to the chick's expanded manaibular pouch. Older chicks pull their parent's head down to the level of the nest. By this method, only one shick at a time receives food. The transition from the first Ieeding method to the second takes about two days and begins between the 5 th and 8 th days of life. Since siblings hatch at one- to two-day intervals, the time-lag between two siblings acquiring the grabbing feeding method confers, for a short period a great advantage on the older chick.

Similar transitions (at 5-10 days) from a pecking to the grabbing feeding method have been observed in Florida caerulea (Neanley 1955), Ixobrychus exilis (Weller 1961), Ardea cinerea (Owen 1955 and Lowe 1954), and Botaurus lentiginosus (Byers 1951).

Soon after all the members of a brood have acquired the grabbing feeding method, severe competition for food begins. There are many sources of evidence for this, one of which lies in the chick's begging behaviour, which is noisy and involves a great amount of movement. Ths components of begging are as follows.

1. One or both wings partly extended and brought forward and upwards (Figure 38), with erratic waving movements which make the chick very conspicuous.

2. Head, neck and the whole body erratically bobbed up and down; beak open; continuous quivering head movements. 
3. Continuous one, two or three-syllabled notes (usually two) which are high pitched in newly-hatched chicks ("zit-zit") but become harsher and more raucous as they grow older. At very high intensities this call is replaced by a harsh squealing.

Very young chicks have poor muscular co-ordination and the above components are weak but in chicks older than about 15 days, all begging movements are violent and a chick chases its parent around the branches, buffetting it and attempting to grab its beak, the adult flapping to retain its balance. A parent usually responds to this by jerking is head upwards beyond the chick's reach and often gives "kok" calls. As soon as onebolus has been passed, the adult walks or flies away from the nest and may remain nearby for several minutes before it is ready to regurgitate again. This avoidance of the chicks is particularly marked with larger broods, the adult spending only about 5 to 10 seconds on the nest while each bolus is passed, and up to 10 minutes perching beyond the reach of the chicks before flying towards them again. Site-attachment tendencies still exist in the chicks, for even when able to fly chicks do not pursue their parent more than about 6 metres.

Although the author has no satisfactory basis for comparison within the family, the begging behaviour of $A$. ibis chicks older than about 15 days: appears extremely conspicuous. This could be interpreted by assuming that competition for food has caused seloction in favour of high intensity begging and the frabbing method of feeding. This explanation is fully supported by Nelson's (1966b) findings in the Sulidae: Sula bassana chicks, which almost always have excess food available, have very weak begging movements and calls, while the young of some related tropical species, which are severely affected by food shortage and commonly starve, have vigorous begging displays with violent wing-movements.

The feeding method of chicks older than 8 to 10 days, namely grabbing the parent's beak, ensures that weaker chicks stand little chance of obtaining food until their stronger siblings have obtained several boluses and are begging less intensely. In addition, the adult's habit of jerking their heads up. and away also gives larger chicks more chance of obtaining food. Finally, it is clear that parents show no individual preferences as to which chick obtains food; the feeding order is decided entirely by the relative sizes and strengths of 
38. phorte of beging.

FIGURE 38. Chick begging

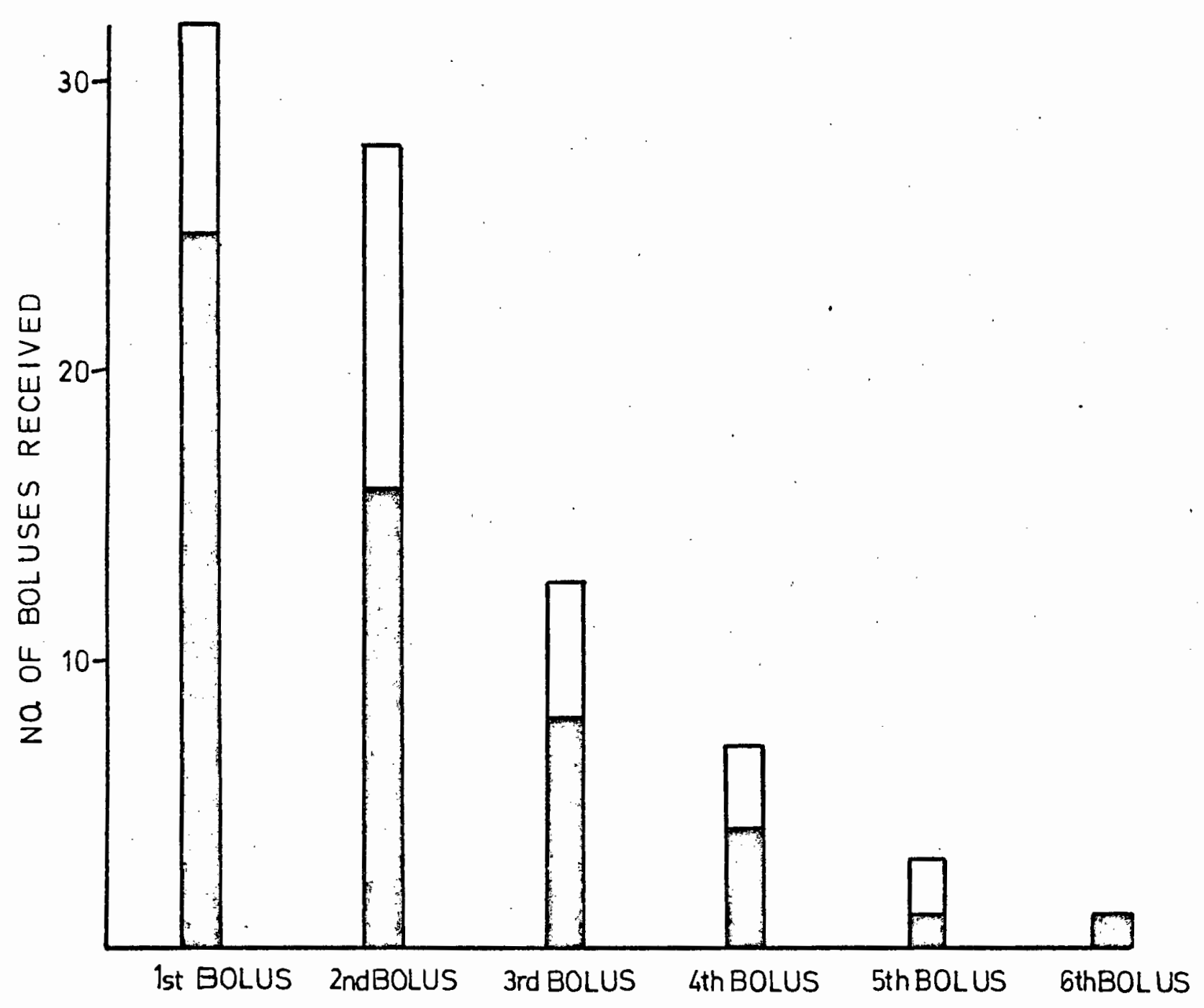

FIGURE 39. Total number of boluses obtained by twins. Solid rectangles: older chicks. Hollow rectuneles: younger chicks. Based on 32 feeds observed in four yairs of twins ranging from 10 to 32 days old. 
siblings, and their dominance relations. Since third-hatched chicks grow more slowly than their older siblings (Figure discussed later), the combined effect of the above three factors is that younger chicks are able to obtain much less food than firsthatched chicks. This is illustrated by Figure 39, which gives the total numbers of boluses obtain in 32 feeds observed in four broods of twins aged 10-32 days. Older sibling obtained $65 \%$ of the total number of boluses (84). Similar figures obtained for triplets are too scanty to warrant presentation, but it is usual for the youngest chicks to starve for long periods, and in several of 9 broods which were weighed daily, the youngest of three siblings failed to gain weight for four successive days or longer.

Fighting and pecking among siblings during feeds is very common, dominance relations being entirely based on their ages. The youngest a chick has been observed to peck its siblings during a feed is at 13 days. Frequently (57\% of 32 feeds), the oldest chick pecks, bites and buffets its sibling and chases it around the nest site, ceasing its attack only when the subordinate chick is well away from the parent. This onesided tighting consits of overt aggression and not ritualised Forwarả Displays. Chicks seldom inflict physical damage on each other, but the attacks effectively reduce the younger chick's chances of feeding. In some broods more than others, this fighting plays a major role during feeds. Chicks never peck older siblings. This rule is inviolable; so much so that in three-chick broods the middle chick only pecks the youngest, although the latter seldom effectively competes, the oldest siblins grabbing most of the food. The point incidentally illustrates that this fighting is not purposive. Siblings never fight like this in the absence of their parents, although a peculiar form of fighting which occurs only in very young chicks is described in section 6.32 .

To conclude this section, the evolutionary effects of competition for food are seen in the chick's violent begging beheviour, grabbing feeding methods, and overt aggression during feeds.

\subsection{Growth rates and chick mortality}

The growth rates of $A$. ibis siblings are chiefly dependent on their relative ages. Figure 40, which records the 


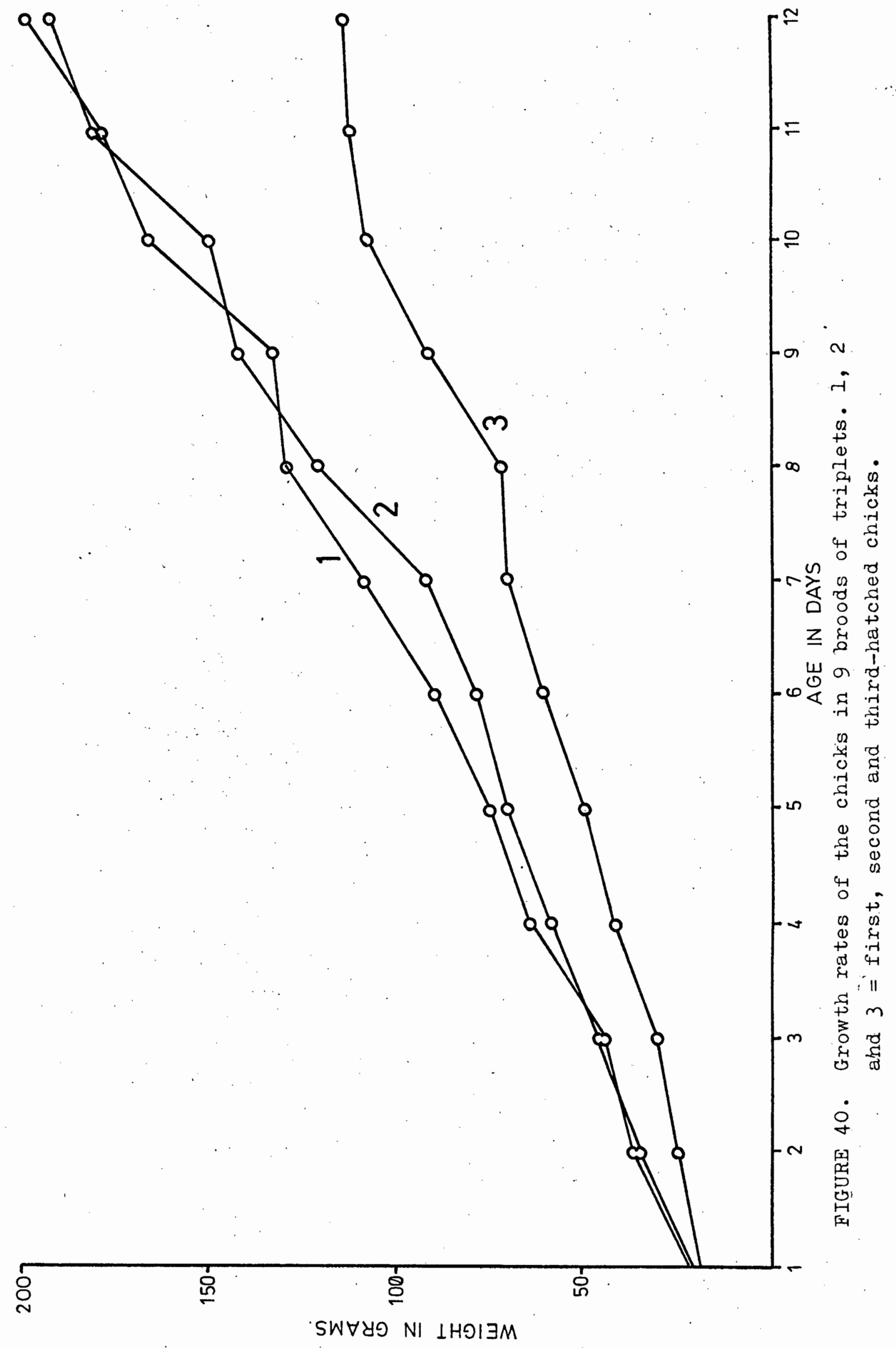




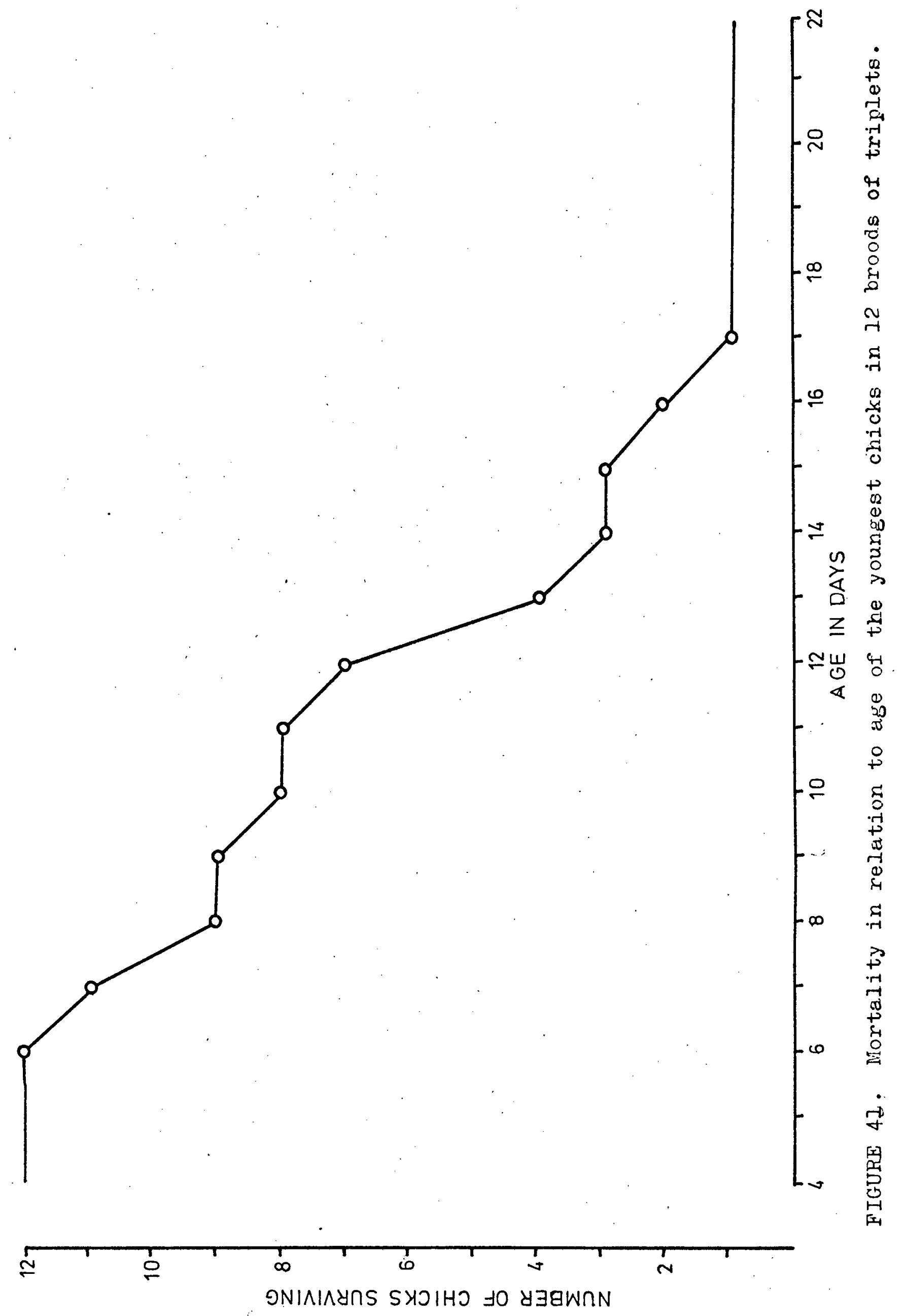


daily weight changes of 9 normal broods of triplets, shows that the average growth rates of first-and second-hatched chicks were almost identical, while last-hatched chicks grew more slowly and their weights never approached those of the others. All weighings were discontinued when the oldest chicks reached 14 days, for most of the 9 youngest chicks starved before this stage, but the figures nevertheless show a. decrease in the average growth rate of the youngest chicks older than 9 days. Owen's (1960) figures for a single brood of Ardea cinerea give a very similar weight-relationship between the younger and older chicks, with the younger two (out of five) dying of starvation at 15 and 20 days.

Starvation is the chief cause of mortality in Ardeola ibis chicks, the great majority of third-and fourthhatched chicks dying before they are 15 days old. Survival rates of nestling could not be obtained by the conventional method of nest-inspection, chiefly because on the approach of a human alI chicks older than about 12 days scrambled out of their nests, many falling to the ground and others becoming stranded in alien nests and subsequently killed by the nest-owners. This was overcome by recording the survival of chicks whose individual histories were known in detail. Observations from a hide of a group of 12 three-chick nests produced daily figures on nestling survival in relation to age. Only one of the first- and one of the second-hatched chicks died, while 11 of the 12 youniest chicks died between 7 and 17 days of age (Figure 4I). None of the 12 nests suffered from predation. The majority of dead and dying chicks found on the ground in A. ibis colonies were emaciated and had clearly starved. The author has never observed three or more siblings older than 20 days in one nest, so the event must be very uncommon. Skead (1966) notes the same pint. Iittle mortality occurs among chicks older than 20 days; tose chicks that do die are usually killed while trespassing, as is the case in Ardea cinerea (Owen 1960).

All published nesting-success figures for Ardeidae (C:en 1960; Teal 1965) are suspect, because the author's experience is that human observers can be a major factor in chick mortality, more so in Ardeidae than in most other groups. Despite tris, Teal's (1965) finding that in 5 species of Ardeidae in the U.S.A. (A. ibis not included) the percentage of chicks dying from starvation varied from 0-10\%, and that predation was the chief cause of mortality, are very interesting. In the 
present study, at least $30 \%$ of A. ibis chicks starved, while predation was relatively unimportant.

Owen (1960) states: "The adaptive value of asynchronous hatching (in Ardea cinerea) is that when food is scarce, the smallest young in the nest die of starvation, but when food is more plentiful all the young are raised."

Siegfried (pers. comm., see below) has evidence that the combined effects of asynchronous hatching and severe competition for food among siblings may be maladaptive in some cases.

Evidence presented so far in section 6.21 and 6.22 indicates that a number of behavioural features have been produced by the selective effects of competition for food. In this category fall: violent begging behaviour; the beakgrabbing method of food-passing; the way in which a chick chases its parent; fighting; and the nestlings' narrow range of bill-colour preferences (next section).

In most bird species, the selective effect of predation places a limit on the conspicuousness of chick feeding and begging behaviour. In a species where predation on the young is negligible, no factors limiting the conspicuousness of chick behaviour can be visualised. (The young of hole-nesting birds such as Picidae have noisy begging calls. In some eagles the older of two chicks always kills the younger (Brown 1966):

In both these groups predation, on nestlings is unimportant). There is little predation on A. ibis young; but more than this, A. ibis and their nests are highly conspicuous in the first place, so that the nature of a chick's begging behaviour could not conceivably influence its chances of being captured by a predator. On this basis, it is likely that no adaptive restrictions have been placed on the evolution of competitions in the form of grabbing, chasing, begging and fighting in A. ibis nestlings. This is supported by evidence that in the crypticallycoloured Ardeids Butorides striatus and Ixobrychus exilis, the young apparently lack begging calls (Cowles 1930 and Weller 1961).

Natural selection in one of its simplest forms exists among A. ibis nestling. There appears to have been unrestricted selection in favour of chicks which during feeds are strongest, most aggressive and beg most conspicuously. This could reach, or has reached, a situation where a selective premium is placed on chicks which destroy their siblings, thus lowering the 
reproductive rate. Ihis conclusion is supported by figures obtained by Siegfried (pers. comm), who found that if the youngest of three siblings survives more than 25 days its growth-rate increases and its weight approaches (but never equals) that of its older siblings. This implies that competition becomes less severe from 20-25 days onwards, enabling the youngest chick to survive.

Examples of maladaptive features are common: the peacock's tail, the size of filter-feeding Cetaceans and chondricthyes and the tracheal respiration system of Arthropods are all evolutionary dead-ends. Nestling A. ibis may constitute one of the few known cases of selection for lower reproductive rates than the species is capable of. (Sula bassana provides one such example; adults lay only one egg but can easily rear two chicks (Nelson 1966a).) If this should be fully proved in A. ibis, it will not in any way contradict Iack's (1954) widely accepted thesis that clutch-size has been evolved to correspond with the largest number of young for which the parents can find food.

\subsection{Significance of beak colour}

Newly-hatched A. ibis have pale flesh-or horn-coloured beaks and lores. From about 5 days onwards the beak begins to darken until it becomes almost completely black at 10 to 15 days, and from about the 30 th day onward it begins to turn paler again until it reaches the normal adult yellow colour at two to three months. Before this colour-change's function can be discussed, it is necessary to present some results on the part played by adult beak colour during feeding. Young nestlings feed bj weakly pecking and grabbing at their parents' beaks, while chicks older than 5 to 8 days grab the beak firmly. Ihe importance of beak-colour as a releaser of pecking was investigated by experiments with models, similar to those performed by Tinbergen and Perdeck (1950) on Larus argentatus.

Series 1 : Methods. New-hatched chicks were not used because they were too dis-coordinated to give positive results, so that the responses measured were influenced by the subjects' past experience to some extent. Chicks 3 to 6 days old were removed from their nests and placed together in a darkened incubator for 5 to 10 hours before testing. All experiments were performed indoors under standardised light conditions. In the first series of experiments, life-sized 
cardboard head silhouettes were used; the beaks were painted red, yellow, green, blue and black and each head had an eye in outline. Each chick was removed from the incubator and placed on a flat, featureless cloth surface, facing away from the light source. The chick would usually look around, then start to give the "zit-zit" begging calls. A model as then presented with the beak held vertical about $2 \mathrm{~cm}$. in front of the chick's beak with tip about $2 \mathrm{~cm}$. above the substrate. The model was always held at right angles to the chick's line of vision and was moved only if the chick turned its head. Each model was presented for 30 seconds and the number of pecks or grabs which struck the model in this time were counted. The 5 models were presented in succession with a 10-second interval between each 30-second presentation. To provide further standardisation chicks were only tested while they were begging and if the subject stopped its "zit-zit" calls the experiment was discontinued.

Series 1: Results. Figure 42 gives the results of 344 pecking responses in 24 tests on 6 individuals. The chick's preference for yellow-beaked models is so obvious that this experimental series was discontinued and more detailed tests planned. One point of note is the small number of pecks ( $5.2 \%$ of total) elicited by the black-beaked model, showing that the degree of contrast between head and beak is of minor importance as a releaser, unlike the situation in Iarus argentatus (Tinbergen and Perdeck 1950) and the black-beaked Egretta garzetta (unpublished)

Series 2: Methods. In the second series of experiments the conditions were exactly as before except that the 5 beakcolours covered only the longer wavelengths: red, orange-red, orange-yellow, yellow and yellow-green. To ensure that the sequences of model-presentation did not affect the results, each of the possible presentation sequences was used once (permutation $5=120$ ). Ten individuals were used, from 7 to 14 presentationseries being performed with each, and a total of 3172 peckingresponses were recorded.

Series 2: Results. Although the number of chicks tested was small, Figure 43 clearly shows that pecking in A. ibis chicks is released by a narrow range of wavelengths, with the mode of the approximately normal distribution slightly nearer "yellow" than "orange-yellow". This wavelength and the wavelength(s) of an adult A. ibis' beak colour could not be measured, but to a person with true colour vision the two 
appeared to co-incide exactly. The distinct bill-colour preference in A.ibis chicks may constitute yet another behavioural response which has been subjected to rigorous selection by competition during feeding. Cullen (1962) gives a number of reasons why it is more likely that a chick's responsiveness is adapted to its.parent's beak-colour, than vice versa.

The colour-preferences obtained for A. ibis were far more marked than those in Iarus argentatus ('inbergen and Perdeck 1950), Sterna fuscata (Cullen 1962), Fulica atra or Gallinula chloropus (Kear 1964), but less marked than those in Larus pipixcan (Collias and Collias 1957).

Following on the above results, the significance of beak colour-changes in A. ibis young can now be investigated. Beak-colour constitutes one of the most conspicuous morphological differences between adults and feathered young. There are many other differences such as degree of feathering, but these change continuously as the chick grows. In several studies (e.g. on Sula bassana Nelson $1966 \mathrm{~b}$ and Nycticorax nycticorax Lorenz 1966), it has been concluded that conspicuous colour-differences between juveniles and adults serve to prevent the young from releasing territorial aggression in their parents. This is not the case in A. ibis. The beaks and lores of four young birds 8 , 15, 20 and 24 days old were painted yellow and some others painted red and blue as controls, but in no case did the adults respond in any way at all to the striking changes in their offspring's appearance. As might be expected, the yellowpainted beaks of the first four experimental chicks were pecked at by the chicks' siblings. These actions were not nearly as vigorous as those occurring during feeding, presumably due to the role played by learning, i.e. the chicks concerned "realised" that their sibling's yellow-painted beak was not a source of food.

On the basis of the above evidence it is concluded that the colour-change to black beaks in A. ibis chicks evolved to prevent the nestlings from wasting energy and perhaps inflicting damage by attempting to eat each other. Supporting this is the temporal coincidence between the onset of beakblackness (5-10 days) and of the grabbing method of feeding (5-8 days). During periods between feeds, chicks younger than about 15-20 days sometimes gently grab a sibling's beak and tug briefly; the most likely interpretation is that chick beaks act as 
suboptimal releasers of feeding behaviour.

In a minority of A.ibis broods (estimated 1-5\%) no blackbeaked stage exists in any of the siblings; the beak changes gradually from flesh-colour to yellow. This is presumably due to a recessive factor but its continued existence, in view of the above conclusion, is probably due to negative reinforcement by which the chicks rapidly learn not to peck each other's beaks.

\subsection{Displays and social interactions of young}

\subsection{Interactions with adults}

At all stages of development (after three days) a chick will threaten all adults, other than its parents, which approach the nest. At three days Forward Display consists only of a forward stab with open beak, and a raspy squeak, but by 7 days the adult component of wing-waving is present and the whole display is better co-ordinated (Figure 35). The call develops gradually from a squeak to a harsh "raa" in about 50 days. Chicks older than about 10 days (the nest is never left unguarded before this stage) are invariably successful in defending their nest ggainst conspecific adults. Although a trespassing adult may threaten in return, it always retreats, and if the contest is more or less evenly balanced, both adult and chick give Head-flick (first seen at three days in chicks). Frequently, younger chicks threaten their own parents; details concerning parent-young recognition are given in Section 6.33 .

In a colonial-nesting species there is always a danger of nests being stolen and their contents ejected (observed twice in $\mathrm{A}$. ibis). The aggressiveness of A. ibis chicks towards trespassing adults may be an adaptive response to this, particulerlir as the nests are not guarded by adults for most of the nestling period.

As described in section 6.14, the chicks tend to wander after brooding ceases, and as a result often walk or accidentally fall onto other nests. Nest-owning adults react very aggressively towards trespassing chicks older than 10-14 days (details later). An adult has few or no inhibitions against attacking a trespassing chick and will peck it as it runs around the nest, squealing and trying to evade the blows, which are always directed at head and neck. Persecuted chicks usually scramble out of the 
nest in time, but if unable to escape are pecked to death. A. ibis adults only attack trespassing chicks and do not "seek out" and attack all unguarded chicks as adult Sula bassana do (Nelson 1966b).

Only once has a chick (23 days) been seen to evict an adult from its (the adult's) nest. The adult returned to find a 23-day chick on its nest, and after a long conflict with many stab exchanges and signs of high-intensity conflict in both birds (crest positions 6 and 9, Head-flick) the adult retreated. Half an hour later the chick walked off the nest and the rightful owner returned.

\section{Young A. ibis possess two (presumed) appeasement} displays. The first is named Facing Away. Occasionally when a trespassing chick is attacked by an adult it does not attempt to escape, but turns its head slightly downwards and away from the attacking adult. "his "type" of movement in which an attacked animal looks away commonly has an appeasement function (cf. Nelson 1965, Tinbergen 1959). In none of the 5 occasions on which it has been seen in A. ibis has the adult's attack been dininished, but Facing Away must be functional in some situations. More frequently, a persecuted chick will crouch flat, immobile, with its whole body pressed against the nest floor. This is named Nest Crouch but may just represent an extreme form of Withdrawn Crouch. On several occasions an attacking adult has been seen to desist completely when its victim Nest-crouched; adults never attack dead chicks in their nests so the adaptiveness of Nest Crouch is apparent.

On five occasions (three different nests) an adult has been seen to feed foreign chicks older than 15 days. In each case, the adult arrived at its nest, its own chicks (14 to 20 days) began begging, then a foreign chick (18, 19, 23 days) jumped down from a nest immediately above, grabbed the adult's beak and fed. In all five cases the adult attacked the foreign chick after a few seconds, and together with its own chicks, drove it from the nest. (The significance of these incidents in parentyoung recognition are discussed later.) This food-stealing is uncommon and plays little part in the species' biology.

Nice (1962) in a synthesis of the development of behaviour in birds, divides a chick's development into 5 stages, the last of which, Socialisation, consists of aggression, flight and self-feeding. A. ibis does not fit into this scheme. Two 
of the four agonistic displays of chicks (Head-flick, Forward Display) are present at 3-4 days, and the other two (Facing Away, Nest (rouch) appear well before the biras can fly. I do not possess sufficient information to decide whether this is exceptional or not, but on the basis of Nice's arrangement, A. ibis shows considerable social precocity.

\subsection{Interactions between chicks}

Except during feeds, siblings rarely fight amongst each other. Most siblings clearly seek each other's company and are held together by some form of social bond, apart from the fact that they share the same nest site. Ihis is shown by the way in which siblings often perch next to each other, in preference to other chicks, even when two or three metres away from their nest. This tendency for a brood to "stick together" "may", be adaptive in that it strengthens nest-defence against trespassing adults. It is not known whether siblings keep company when they wander further from the nest site.

The aggression which occurs during feeds has already been described. Siblings younger than about 8 days occasionally fight in a stereotyped manner. Opponents sit upright, facing each other, and repeatedly lunge forward, pecking each other's body and head. Although contests may last up to 20 minutes, they cause no damage other than small tears of the facial skin. No cause or function for these contests can be suggested, for they do not occur during feeds and may continue ever when both chicks are removed from the nest.

Young A. ibis give 5 types of calls; the raspy squeak of Forward Display; begging calls; fear-squeals when attacked and "eeeh" discomfort notes. The latter call is not given after 10-12 days; the others continue throughout the period in the nest. The fifth type of call in chicks is the "Chirp" phrase, which consists of two to about 15 distinct notes, variable and difficult to describe. Individual notes vary in pitch from high chirps to low croaks. Chirp's comonest form is an ascending followed by a descending series of notes, but Figure 44 gives a rough idea of the wide range of variation.

Chirp calls usually accompany one of two movements. In $60 \%$ of 50 observed cases (Table 8), the calling chick extended its neck upright which gave it binocular vision of its sibling. In $14 \%$ of 50 cases the calling chick crouched into a 
TABLE 8. Contexts and accompanying movements of Chirp calls. (Total 50 observed calls.)

\begin{tabular}{|l|c|c|c|c|}
\hline \multicolumn{1}{|c|}{ situation } & \multicolumn{2}{|c|}{$\begin{array}{c}\text { accompanying movements } \\
\text { extended } \\
\text { neck }\end{array}$} & crouch $\begin{array}{c}\text { no } \\
\text { movement }\end{array}$ & total \\
\hline $\begin{array}{l}\text { older chick Chirps } \\
\text { to younger, no apparent } \\
\text { cause }\end{array}$ & $6 \%$ & - & 2 & 8 \\
\hline $\begin{array}{l}\text { younger chick Chirps to } \\
\text { older, no apparent } \\
\text { cause }\end{array}$ & 12 & 6 & 6 & 24 \\
\hline $\begin{array}{l}\text { chick approaching } \\
\text { nest and sibling }\end{array}$ & 26 & 2 & 8 & 36 \\
\hline $\begin{array}{l}\text { to an approaching } \\
\text { chick }\end{array}$ & 8 & 2 & 2 & 12 \\
\hline other & 60 & 14 & 26 & $100 \%$ \\
\hline totals & 8 & 4 & 8 & 20 \\
\hline
\end{tabular}
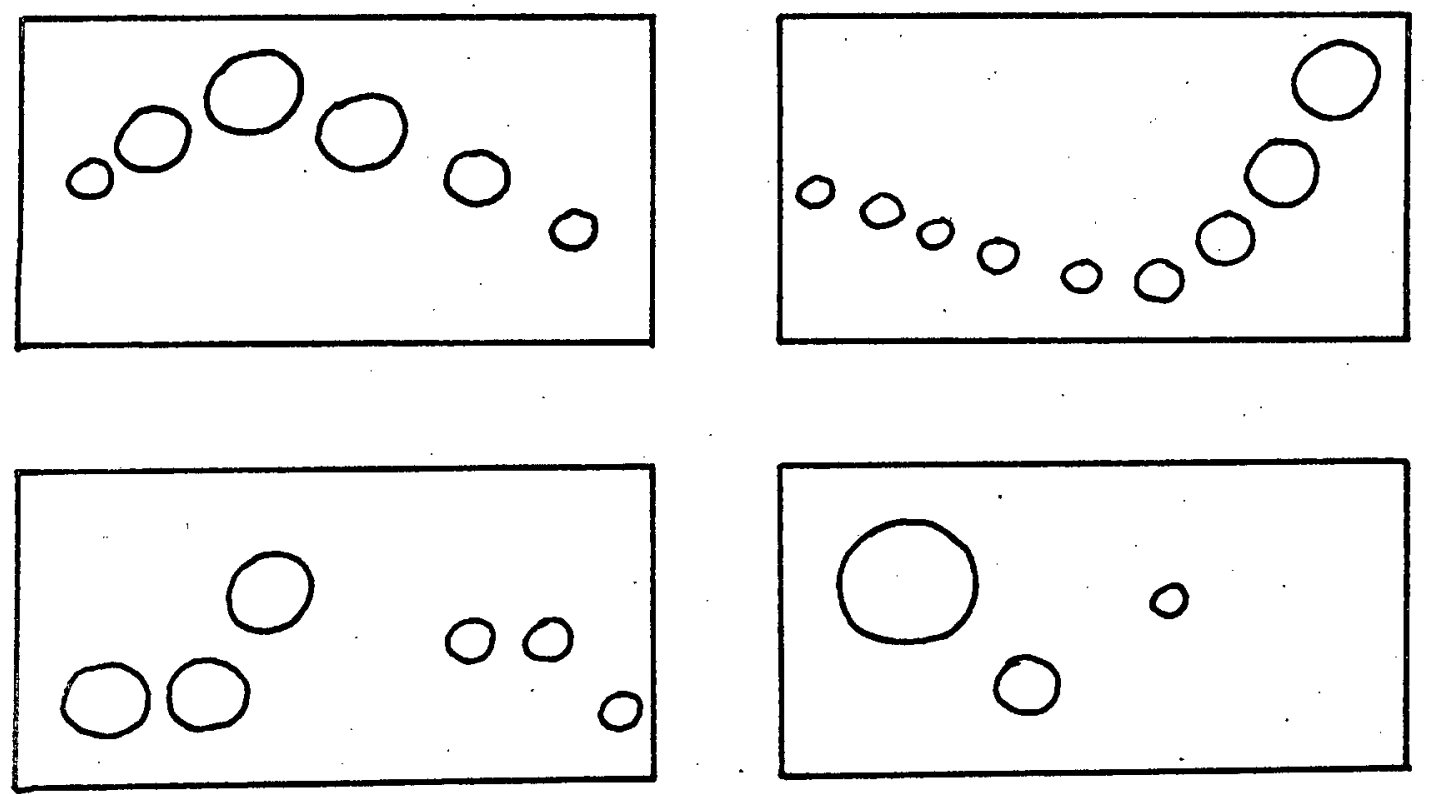

FIGURE 44. Four examples of Chirp calls. Abcissa: time (approx. 2 secs.). Relative frequency. Size of circles is approxomately proportional to the relative intensity of individual notes. 
position similar to Withdrawn Crouch, and sometimes there were pumping up-down movements between the two positions. Table 8 shows that the greatest number of Chirp calls were given by chicks as they approached their siblings on their nests; i.e. in a greeting situation. The next commonest category was calls spontaneously given by the younger of two siblings. In the greeting situation it is likely that the call serves to prevent any possible aggressive nest-defence behaviour by the chick on the nest, and to signal non-aggression by the chick approaching the nest. This suggestion is feasable since outside the brood, Chirp calls only occur among "friendly" chicks (details below). Chirp probably also facilitates individual recognition, for in $24 \%$ of 50 cases, the chick which was Chirped to looked up and gave similar calls in reply. Lowe (1954, p. 90) mentions calls in young Ardea cinerea which may be similar to Chirp.

Chirp calls have an interesting development. First heard at three days, the phrases become longer and more complex over the next 20 days. At about 25-30 days, the previously haphazard series of notes are replaced by Chirp phrases in which can be recognised the pattern of Rick-rack...... Chatter which is characteristic of adult greeting ceremonies. By 40-50 days these adult calls are clearly recognisable in all chirp phrases. The transition is innate, for it also took place in tame chicks removed from the colony at 14-15 days and reared in isolation from adult birds. However, elements of learning/. imitation must be present, as the Rick-racks of hand-reared birds had not become exactly the same as wild adults' by four months age (time of writing). The chief point of interest is that although chicks' Chirp calls alter out of recognition during development, their derivatives continue to be given in the same contexts in adult birds.

The behaviour of siblings having been described, interactions between chicks from different broods will now be dealt with. Trespassing chicks are usually threatened and/or pecked by any other chicks whose nest-territories they may happen to cross. Chicks are however definitely less aggressive towards each other than they are to adults. Chaiks have never been observed to kill or badly damage trespassers, but on two occasions chicks (14, 17 days) which wandered onto adjacent nests were severefy pecked when the resident chicks (25-30 days) returned. Normally, a chick will avoid another nest 
occupied by older chicks.

Prolonged contests over nest-ownership have been observed between chicks. A2 (a twin, 26 days) walked onto nest $\mathrm{C}$ and was threatened by C (a singleton, 17 days). A2 retaliated and a long pecking fight ensued, followed by a three-minute tugging contest.with locked beaks. C then submitted and performed Facing Away, but the attack continued. C then Nest-Crouched flat on its nest, and $A 2$ ceased its pecking and relaxed until C moved again. A2 left after about half an hour.

In a series of tests concerning parent-young recognition, the appearance of a number of chicks older than 14 days was altered in various ways (details in section 6.33). In only one case did the experimental chick's siblings respond in any way to the changes; the chick with the red-painted beak was pecked a few times by its older sibling. The absence of any marked response in these experiments suggests that chicks recognise each other by subtle behavioural characters.

Young $A$. ibis are not equally aggressive to all trespassers. Occasionally a brood will accept and tolerate an individual from another nest, and two individuals from different nests may prefer each other's company to that of their own sibling's. Two such cases were observed in detail in colourringed chicks and one is described here.

\section{Nests A contained twins and $B$ ( 1.5 metres away)}

contained a singleton, all three chicks being the same age within 4-5 days. From the ages of 18-20 days onwards, chicks Al and $B l$ spent most of their time in close company for as long as they remained in the nest tree, usually perched within $50 \mathrm{~cm}$. of each other. They preferred nest $B$ but sometimes perched on $A$. The two chicks behaved as siblings and often gave chirp calls when one joined the other. There was never any aggression between BI and $A 2$ (younger sibling of $A 1$ ), but $A 2$ never perched close to $B I$ and always walked off when BI approached nest A. A2 never sought the company of any chick other than its sibling (which in any case preferred the company of $\mathrm{Bl}$ ), and perched alone. This liaison did not affect the parent-young relationships, for the $B$ adults would attack $A I$ if it was found trespassing; i.e. the chicks continued to be fed by their own parents. The chicks. apparently tolerated each others' parents, for BI allowed the A siblings to be fed on nest $B$. Once, when female $B$ returned and attacked Al for trespassing, Bl excitedly threatened the "friend" 
but this was exceptional.

The above situation did not arise because BI was a singleton, because in the other similar case studied in detail, two sets of twins were involved, and because most singletons never seek the company of any other chick. No social cause or biological function can be seen for these unusual relationships. All that can be said is that chicks sometimes develop close "friendships" for individuals from other nests. Noble, Wurm and Schmidt (1938) recorded quite complex interactions in caged young Nycticorax nycticorax. Two significant points emerge. Firstly, older chicks clearly recognise their siblings, and some also distinguish between "friends" and strangers. This does not occur in adults. Secondly, and correlated with the above, interactions among chicks are far more complex than those of nesting adults.

\subsection{Parent-young recognition}

liethods: In a series of tests designed to investigate at what age parent-young recognition develops, chicks of different ages were placed in nests other than their own. If the chick was driven from the nest or killed by the foster-parent within 5 minutes it was noted as "rejected", and all other responses were noted as "accepted". (It is unlikely that a j-minute limit affected the results because in all cases the chick was attacked within two minutes, if at all.) Nelson (1966b) makes the point that one can never show that a parent does not recognise its young, in the sense of receiving a unique optical pattern of stimulation, but only that it will react favourably even to a strange chick. Ages of introduced chicks and the average ages of the foster-nest chicks ranged from 2-20 dajs, (2-day intervals. This necessitated 100 interchange experiments. Only foster-nests with two or three siblings were used.

Results: Introduced chicks 2-10 days old were accepted by all foster-parents, regardless of the age of the foster-parents' brood. Three of the 12-day, 8 of the 14-day and all of the 16-day introduced chicks were rejected by their foster-parents. The inference is that introduced chichs 12-14 days or older are treated as territorial intruders, and that chicks 10 days or younger are not. Unless it was significantly you: er than the foster-parents' brood and could not compete, an int:uduced chick 10 days or younger was fed. 
Rejection behaviour towards foreign chicks has evolved in many colonial species, presumably because it is disadvantageous for chicks to beg from adults indiscriminately; acceptance could result in inadequate feeding for all. Parent-young recognition has been recorded in Spheniscidae (Sladen 1953, Richdale 1957, Stonehouse 1960, Pettingill 1960), Diomedeidae (Rice and Kenyon 1962), Laridae (Tinbergen 1953b) and Rallidae (Alley and Boyd 1950). Usually; only young above a certain age are distinguished by the adults. In A.ibis, the development of rejection behaviour in adults approximately coincides with the stage when brooding ceases and the young begin to leave the nest.

Attempts were made to discover the basis of parent-young recognition and to answer the questions: (a) Do adults recognise their young individually and the young base their responses on the adult's behaviour, or vice versa? (b) On what behavioural or structural features is recognition based.

The first question was partly answered by observation. older chicks clearly recognise their parents, for when an adult alights in a nest tree, its young usually start begging immediateIy, before the adult moves towards them. Skead (1966) noticed that chicks would start begging even before their parent had landed. Rarely, older chicks beg at a strange adult nearby, but always subside rapidly. Young chicks often weakly threaten their parents, but recognition improves rapidly as this has not been observed after 17 days .

Evidence for an ability of parents to recognise their young is mixed. Parents always attack trespassing chicks older than 10-14 days (details above), and very rarely attack their own chicks, but this does not in itself prove that parents recognise their young. Figure 45 illustrates diagrammatically the 6 possible types of chick behaviour when an adult returns to the nest, and the adult's responses. All comments are confined to chicks older than 14 days. Most observations are based on natural experiments created by chicks trespassing.

1. The chick runs away from the adult. Only trespassing chicks do this, and they are invariably attacked by the adult whose nest they are on. Not possible to state whether chick ran away because adult showed signs of aggressiveness, or whether adult became aggressive because chick showed signs of fear. 


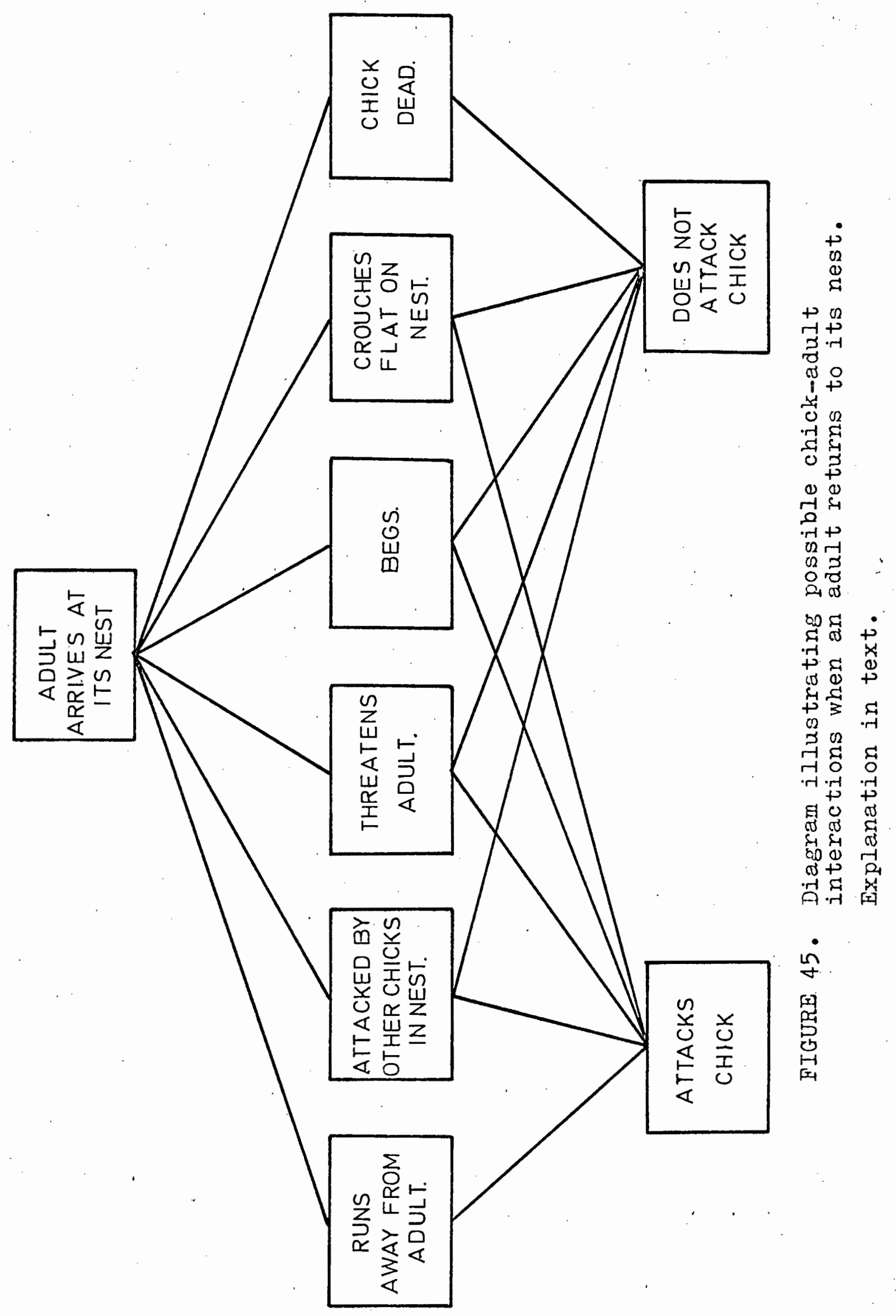


2. Chick attacked by the other chick(s) in the nest. This commonly occurs between siblings during feeds, but the adult never pays any attention. Only when the persecuted chick was a foreigner, was it attacked by the adult. Not possible to decide whether adult's response represents true recognition, or whether it is based on the foreigner's treatment by the other chick(s).

3. Chick threatens the adult with a Forward Display. Adult does not respond if the chick is its own, but always attacks if the chick is a foreigner. Suggests true recognition by adult, but chick may have shown signs of fear not noticed by observer.

4. Chick begs (usual response). Only twice has an adult been observed to briefly attack its own begging chicks. on all occasions (about 7) that a chick has been observed to beg from the "wrong" adult in the "wrong" nest, it was attacked after a few seconds. Suggests true recognition, but chick may have whown signs of fear not noticed by the observer.

5. Chick crouches flat on nest. Only trespassing chicks will do this. Chick usually ignored as long as it remains crouched, but on one occasion an adult was seen to peer very closely at a Nest-Crouching foreign chick before attacking it. Suggests true recognition by adult.

6. Chick dead: always accepted and brooded, whether it is foreign or was one of the adult's offspring. In these cases, adult non-aggression may be a result of the chick's inactivity. (In Sula bassana dead foreign chicks are attacked, Nelson 1966b).

In none of the first four situations listed above is it possible to decide whether the adults actually recognise their own chicks, or whether trespassing and resident chicks behave differently and the adults' responses are based on the chicks' behaviour towards them (the adults). In the fifth case recognition probably occurred. In the sixth case, adults probably do not recognise their young.

Concerning the second question: (b) "On what behavioural or structural features is recognition based", recognition of young by their parents was examined further. Experiments were performed in which different chicks, ranging from 14 to 23 days age, had their appearance altered. Different body regions were either clipped or painted; painting had the additional effect of making 
the feathers flat and bedraggled. The following alterations were made: crest painted blue; crest feathers clipped; beak lores and circumorbital skin painted blue; ditto painted red; blue stripe painted.behing eyes; blue ring painted around eyes; cardboard "comb" glued on beak; feathers on ventral side of head clipped; entire neck painted blue; clipped back feathers, exposing skin and quills; ditto wing coverts; entire plumage painted pink. In none of these cases did the parents respond in any way to the drastically changed appearance of their young. Except for the legs, no body region was changed in both colour and structure and appearance, i.e. recognition of young by their parents (if it occurs) cannot be based on structural features.

While the above experiments provide some comic relief, they do not inform us how parent-young recognition occurs. Voice is not a critical factor because: (a) chicks do not always give beggin calls when their parents arrive, (b) adults will attack foreign chicks which have not uttered a sound, (c) dead chicks are always accepted, (d) chciks usually beg at a newlyarrived adult long before it gives any calls.

In conclusion, there are two possibilities. (a) Since adults do not respond to bizarre changes in appearance of their young, they may recognise their young by subtle behavioural characters. (b) Since chicks definitely distinguish between parents and strange adults, it is possible that an adult's responses are based on a chick's behaviour towards it. 
The chief purpose of this section is to provide descriptive material for any future taxonomic revisions of the family Ardeidae, so only points of difference between the three species' behaviour have been elaborated here. In addition, comparisons between $\underline{E}$. garzetta and the morphologically very similar E. thula are made.

\section{I The Behaviour of Egretta garzetta.}

\subsection{Maintenance activities}

E. garzetta possess a wide range of complex feeding patterns, all of them very similar to those Meyerriecks describes for E. thula and Dichromanassa rufescens. E. garzetta is purely aquatic and has never been observed to feed on dry land. When feeding, the birds spend most of their time walking through the shallow water with quick, jerky paces, but sometimes the movements are slower and more recognisable as the basic Ardeid Wade or Walk Slowly feeding method. E. garzetta seldom feeds by standing motionless in the Stand and Wait position. A common behaviour pattern in E. garzetta is foot-stirring, in which a bird walking slowly through shallow water extends one leg forwards and vibrates it underwater for a few seconds, usually peering at the surface afterwards. The pattern is very common in E. thula and Dichromanassa mufescens (Neyerriecks) and Melanophoyx ardesiaca (Brockhuysen pers. comm.) and it has been suggested by several authors (e.g. Meyerriecks 1959) that the feet act as a lure for carnivorous prey, but no experiments have been performed.

Thirty-second observations on 5 different individuals gave an average flapping rate of 183 beats/mins. for normal flight in E. garzetta. The description of comfort movements for A. ibis applies equally to this species.

\subsection{Forward Displays}

In appearance, causes and contexts, Forward Display in E. Saraetta is very similar to A. ibis'. All 10 components listed in section 3.21 occur in E. garzetta; the most noticeable difference between the two species being the relatively stronger S-curve of the anterior part of E. garzetta's neck (figure 46), and the beak which points downwards at an angle of about $45^{\circ}$. 
FIGURE 46. Forward Display in Egretta garzetta

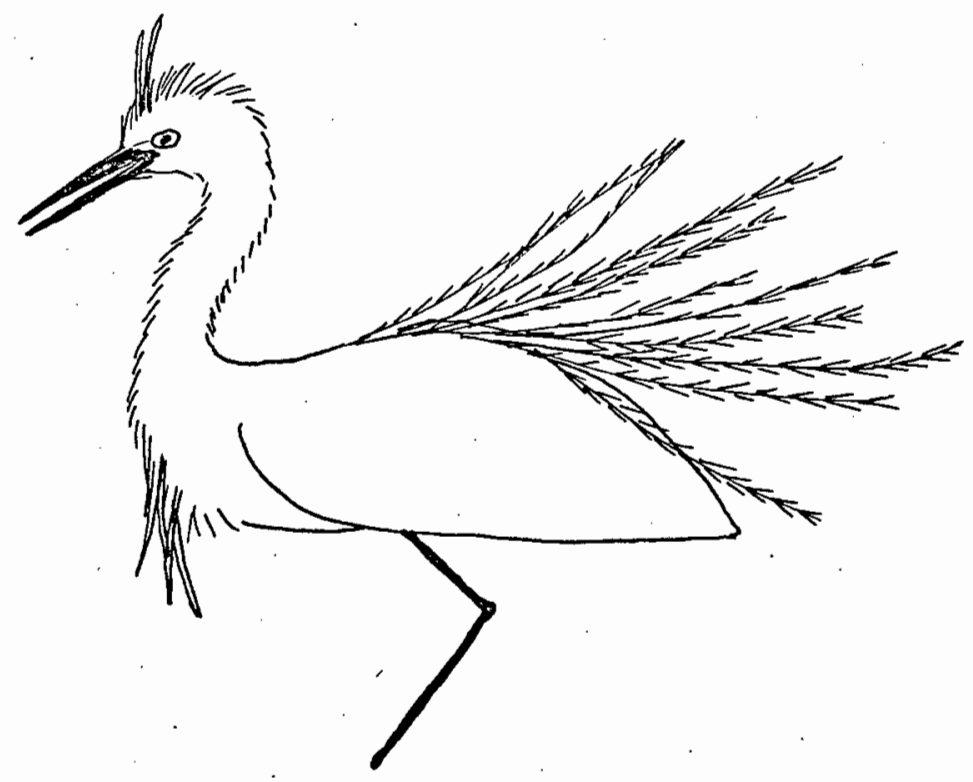

FIGURE 47. Extended Neck Flight in Egretta garzetta

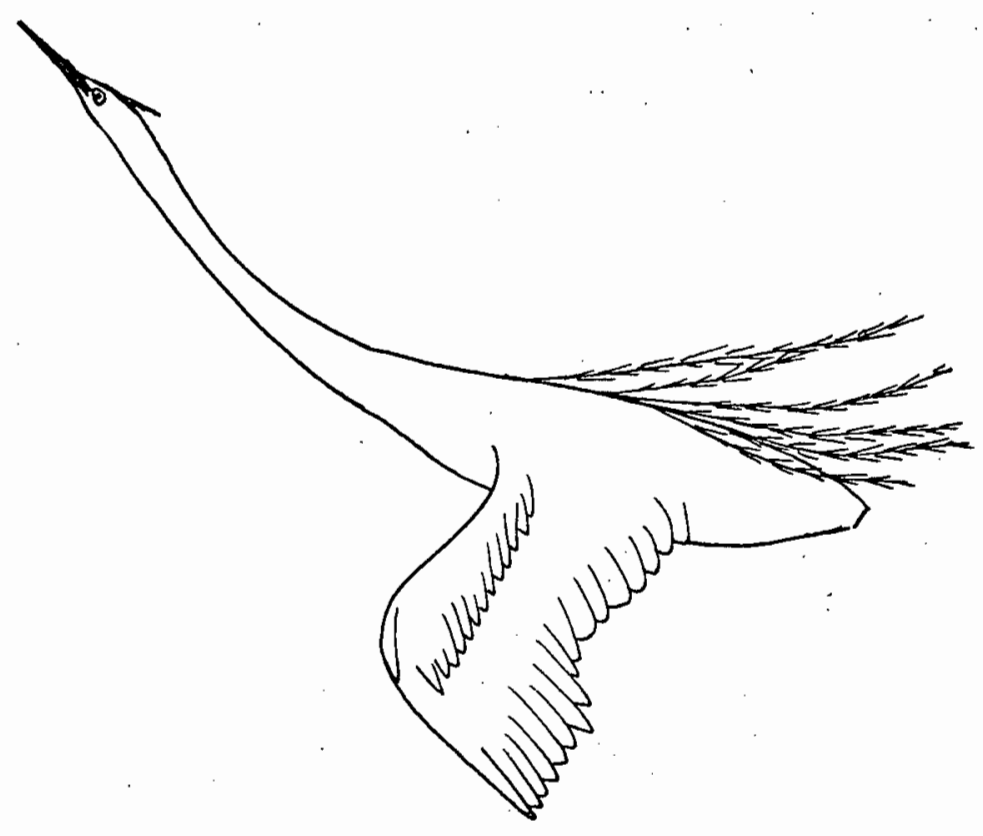


The accompanying call is a short, harsh gargling note "growp". The two long lanceolate crest plumes in E. garzetta behave in the same way as the rest of the anterior crest region and not as a separate feather unit. Fights are very frequent, particularly during the early stages of the breeding season. The clashes are more severe than in the other two species and fight ing birds often fly upwards and continue to peck and buffet while in the air; this corresponds very,closely to Meyerrieck description of fighting in E. thu].a.

\section{$7.13 \quad$ Calls}

Apart from the sounds accompanying Forward Display, Stretc and Twig Shake, E. garzetta has 6 loud calls. As in the other species, two are common in greeting ceremonies: a two-syllable call rendered "da-Wah" in $\mathrm{E}$. garzetta (second note stressed) ar a stammering call (Chatter). Two calls are very common durins the Flying Around stage in unmated males: a long gargling cal: and a brief, hollow call, rendered "Dow" (sometimes "wa-Dow"). Both these calls are given when the bird is perched upright, ar have no apparent immediate effect on other individuals, but presumably act as advertising calls. A third call. of uncertaj context and function consists of a descending series of 5 to 9 hollow notes which do not accompany any visixal display.

E. garzetta has a distinct flight call: a long "aaaah" note gj as the bird flies away from its perch or nest, and is also common in feeding birds at all times of the year

\subsection{Snan Display and Twig Shake.}

Snap Display has not been observed in this species, but th does not rule out the possibility of it beine present. Twig Shake is uncommon: unmated males perform low-intensity Twig Shake movements very similar to those described for $A$, ibis, accompanied by a brief nasal stammering call homologous to Nase Chatter in A. ibis.

\subsection{Flight Displays}

E. garzetta performs at least three types of flight disple The first, named here Circuit Flight, may be homologous to the Circle Flight Displays described by Nejerriecks; it consists c a flight 30 to 300 metres in length in which the bird returns its original perch or nearby, differs from normal flight only i that the neck is partly to fully extended, and apparently occur 
FIGÜRE 48.' Stretch Display in. Egretta garzetta

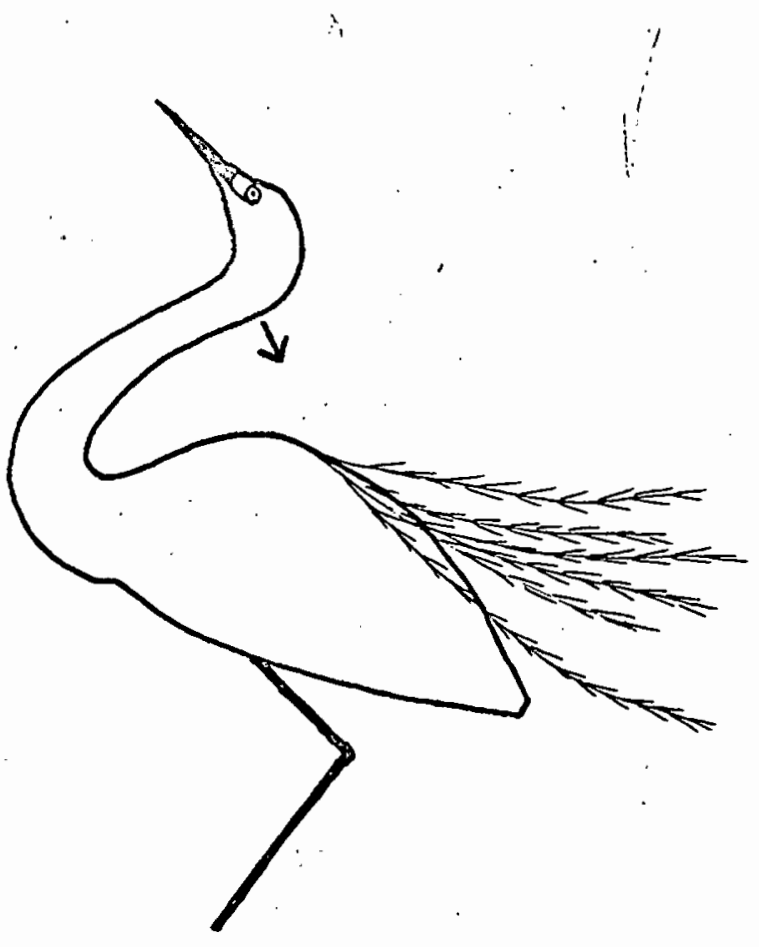

FIGURE 49. Neck-extended position in greeting ceremony in E. garzetta

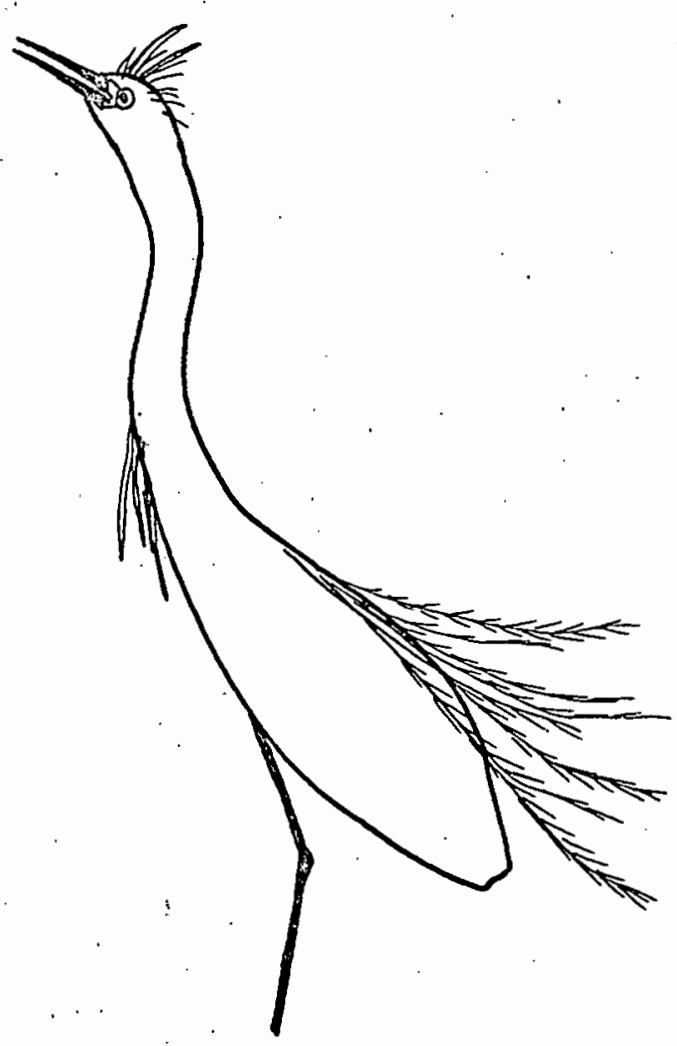


only in unmated males. Flap Flight Display, which occurs both in unmated males and in recently-paired birds of both sexes, is very similar to the same display in A. ibis, except that the "thud". wing-sounds are weak and sometimes absent. The head, neck and leg positions are the same in both species. A distinct display in E. garzetta, named here Extended Neck Flight, was not described by Meyerriecks. For about 5 to 10 metres after the takeoff the displaying bird flies with neck fully extended about $20^{\circ}$ above the horizontal, head about $30^{\circ}$ above the horizontal (figure 47), and scapular plumes raised.

\subsection{Stretch}

Stretch is restricted entirely to unmated males, where it is infrequent. Head and neck are partly extended forwards and upwards at an angle of about $60^{\circ}$ before being brought backwards and downwards (figure 48), scapular erection occurs and leg movements are very slight. The movement of the head and neck is short and jerky, completely unlike the slow, full movement in A. ibis. A brief gulying call "ow" is given simultaneously with the backward movement of the head. Stretch in E. garzetta is totally different to the Stretch of E. thula (Neyerriecks), so there is no point in drawing a detailed comparison.

\subsection{Greeting Ceremonies}

The first response of a sitting bird to the arrival of its mate is to stretch its head and neck upwards (figure 49), probably to provide binocular vision of its mate, (a discussion of the subject appears in section 3.3), giving "da-Wah" and Chatter calls. Both birds then adopt an attitude very similar to medium-intensity Forward Display (figure 46) with full erection of all plumes. Although "impressions" do not constitute satisfactory scientific evidence, greeting ceremonies in both the Egretta species give the strong impression of involving far less conflictand excitement than they do in A. ibis. Apart from this, there are three very fundamental differences between greeting ceremonies in $\underline{A}$. ibis and the two Egretta species. Firstly, crest-erection is full in Esretta but absent in A. ibis, which implies that the crest has different signal values in the two genera. Secondly, complete Stretch Display is never present in Egretta sreeting as it is in A. ibis, although the neck-extended movement may correspond to the first phase of stretch. In section 3.2 it was concluded that stretch in A. ibis greeting 
ceremonies served as a signal of non-aggression, and its absence in Egretta show that there has been no selection in favour of this in Esretta. Thirdly, greeting in A. ibis usually involved close bodily contact between the pair, whereas in Egretta greeting birds seldom touch each other.

\subsection{Bill-clappering}

Bill-clappering, which occurs in many Ardeidae (Hudson 1962), is common in both sexes of E. garzetta, particularly during greeting ceremonies. The only invariable component of Billclapper is rapid movement of the mandibles which produces a rattling sound. Displaying birds may put their neck across their mates', or perform the clappering movements several centimetres above their mates' backs or the display may be undirected, the bird Bill-clappering while in the usual hunched perch position.

\section{I.19 Head Flick, Wing-touch, Wing Spread}

Head-flick movements identical to those described for A. ibis occasionally occur. The display's apparent rarity may be due to the fact that the E. garzetta nests were greatly dispersed among the A. ibis nests, so that interspecific encounters between E. garzetta were seldom observed. Wing-touch, as described for A. ibis, has been observed on only five occasions, when it appeared less "crisp" and less ritualised than it is in that species. Wing-spread, exactly as described for A.ibis, occurs in unmated and newly-mated male E. garzetta. These descriptions and comments apply equally to E. intermedia

\subsection{Breeding behaviour}

At the Paarl colony in 1966 the first E. garzetta arrived between the 8 th and 15th August, the first egg was läid about the 20 th August and the last between the loth and 16 th November. All unmated birds are very mobile compared with A. ibis, and spend a great deal of time flying around among the nesting trees and making numerous Circuit Flights and Flap Flight Displays. Aerial hover-fighting (sexes undetermined) are frequent and unmated birds frequently give the long gargling notes and "Dow" notes already described. This presumably corresponds to the "Flying Around" stage Meyerriecks describes in three of the species he studied, and indicates that unmated E. garzetta possess weak site-attachment tendencies compared with A. ibis at the same stage. 
FIGURE 50. Copulatory attitude in Egretta garzetta

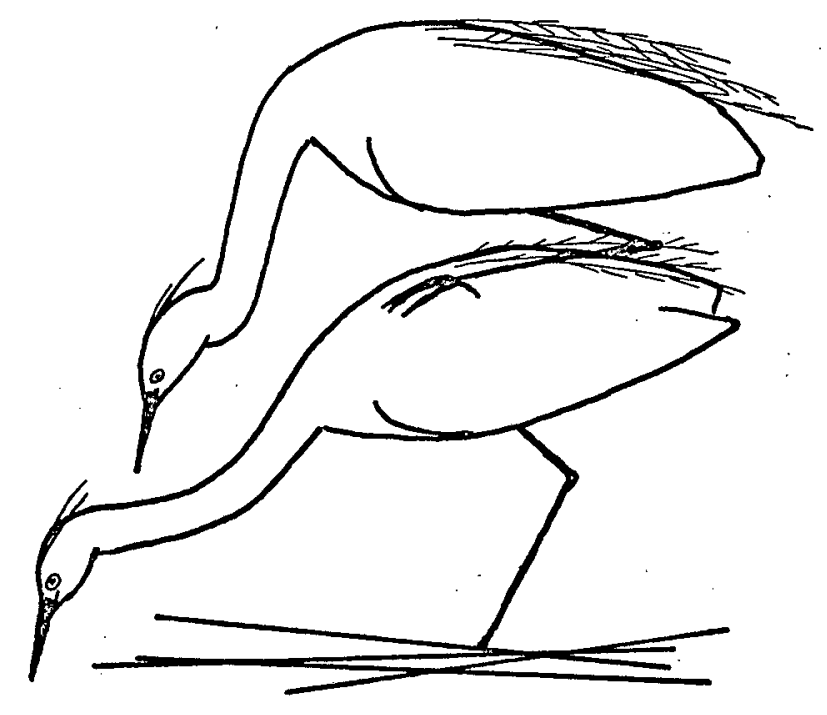

FIGURE 51. Snap Display in Egretta intermedia

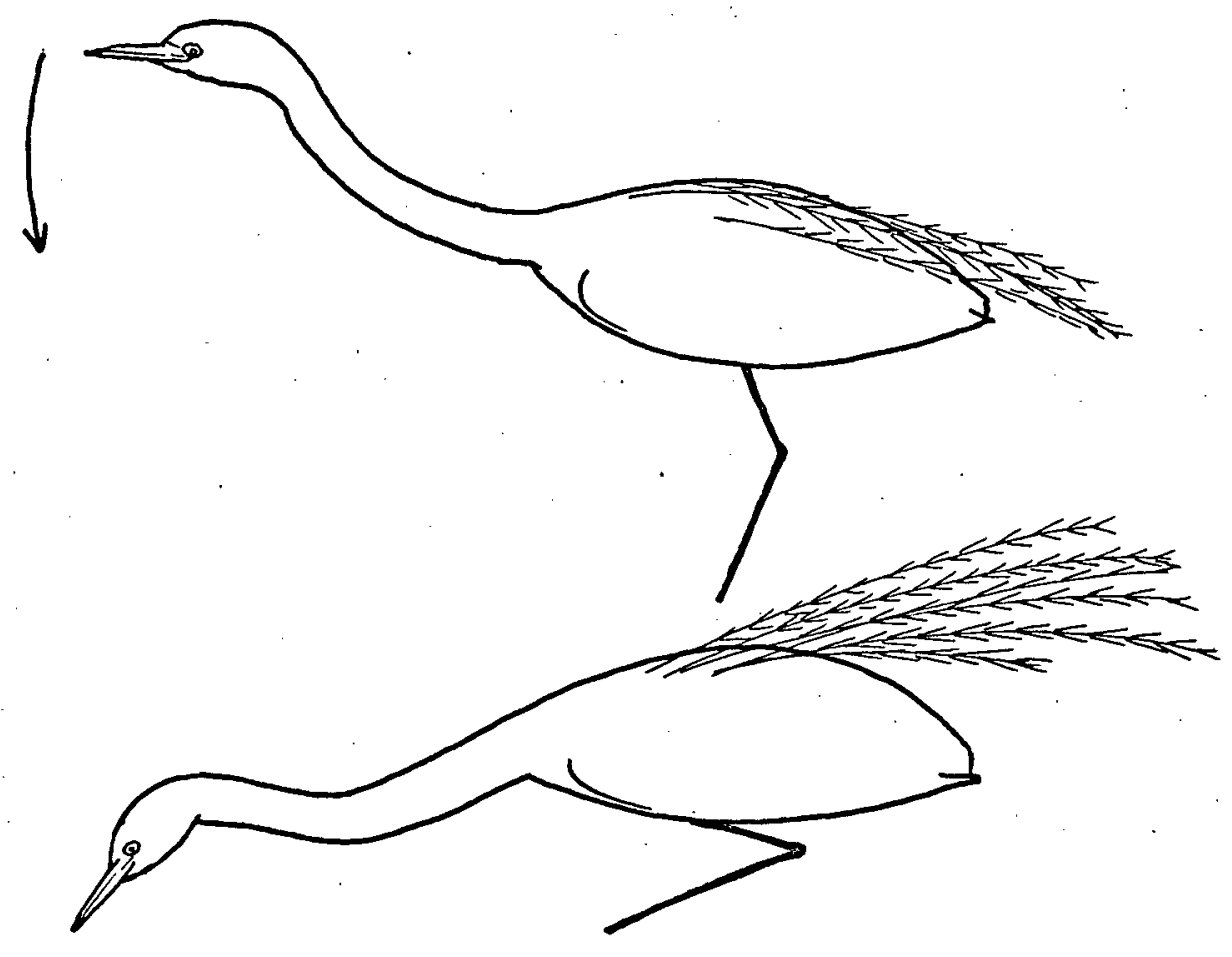


Apart from the bird's great mobility, the processes of mate-selection and pair-formation are similar to A. ibis. Males adopt and aggressively defend small territories against all conspecifics, and give many loud "Dow" calls, which must have an advertising function. These males spend much time walking up and down their sites, but Circuit Flight and Flap Flight Display remain common. Females are strongly attracted to màles behaving in this way and perch nearby, peering and craning in the peculiar long-necked attitude already described for A. ibis. Several males and females may be attracted, females repeatedly approach the males and are supplanted in turn, and in the course of all this activity the whole group moves around the colony, sometimes over a distance of 50 metres or more, many of the birds (sex unknown) giving gargling and "Dow" calls. The actual moment of pairing has not been observed.

All the above corresponas very closely to Meyerrieck's description of $\mathrm{E}$. thula's behaviour: "the Snowy Egret appears to have an ill-defined territory, at least during the first few days. Typically, one male begins to advertise from a conspicuous song post and is joined by several males and females. A bout of calling back and forth ensues, and the little party of advertisers moves about, not settling in one particular spot for very long."

During the pair-formation stage in E. garzetta both sexes perch together on the nest for long periods, but seldom in bodily contact as A. ibis do. Bill-clapper and Flap Flight Display are common activities and greeting ceremonies occur every time one bird arrives at the nest. As in the other two species, the male brings nesting material while the female actualiy builds the nest.

The female copulatory attitude (figure 50) is completely different from that of A. ibis, for the female almost stands, with the angle between the tibiotarsus and tarsometatarus varying from $100^{\circ}$ to $160^{\circ}$, exactly as in Strijbos' (no date) photograph of copulation in Ardea cinerea. The corresponding angles in A. ibis are $20^{\circ}$ to $80^{\circ}$. This may be of considerable taxonomic significance. Copulatory attitudes are among the most phylogenetically stable of all features, for within any one group they fulf1l exactly the same requirements, so adaptive radiation is minimal. In the present case the feature is all the more significant, for Egretta has proportionately longer legs, so might be expected to squat lower than A. ibis, yet the 
reverse occurs.

\subsection{The Behaviour of Egretta intermedia}

\subsection{Miaintenance Activities}

The species' chief feeding method is the widespread Ardeid pattern Wade or Walk Slowly (Meyerriecks); the bird slowly and steadily paces forward, in an upright position with neck extended, suddenly lunging downwards when prey is sighted (E. intermedia is primarily aquatic and $68 \%$ of the 38 feeding birds seen in two years were wading in shallow water and the remainder in pasture near water). Another feeding method, infrequent in this species and absent in the other two, consists of hovering just above the water's surface, particularly if it has a dense growth of Potamageton; the bird actually walks across the surface, pecking at prey it sights. This probably does not correspond to the Hover-stirring movements described by Meyerriecks (1959), for the birds are simply trying to support themselves and do not perform leg-stirring movements.

Four 30-second observations on different individuals gave an average of 162 beats/min. for normal flight.

\subsection{Forward Display}

Except for a few details, the description and illustration for E. garzetta apply for this species as well. E. intermedia lacks specialised pectoral and crest plumes, but the crest movements are the same as for the other two species. In place of the harsh calls in the threat diaplays of the other species, E. intermedia has a weak, buzzing note (see below).

\subsection{Calls}

All calls in this species have a quite distinct tone, differing from those of all other Ardeidae described so far. All except the flight call are very faint with a hoarse buzzing quality, impossible to describe onomatopoeiacally. Apart from the calls associated with Forward and Stretch Displays, three calls were distinguished. Two occurring in greeting ceremonies are clearly homologous to "Rick-rack" and Chatter in A. ibis and "da-Wah" and Chatter in E. garzetta: the former is a faint, buzzing two-syllabled call and the latter, although very faint, has a staccato stammering quality similar to Chatter in the 
other two species. Exactly the same call sequence and contexts during greeting exists in all three species, all birds giving first the two-syllabled and then the chatter calls. On one occusion an E. intermedia flying away from its nest gave a deep two-syllabled croak, but otherwise no flight calls have been heard. No alarm calls have been heard. The differences between the calls of E. intermedia and those of all Ardeidae so far studied are very striking, and must have some clear-cut function. E. intermedia's only congener in Africa, E. garzetta, has a wide range of raucous calls, so the most likely explanation is that there has been strong selection for specific distinctiveness of auditory signals: between the two species.

\subsection{Twig Shake}

Althoush far less frequent than it is in A. ibis Twig Shake in E. intermedia is very similar in most respects, except that no accompanying call has been heard. The "down" position bears a resemblance to Snap Display, but there is no overlap, for Snap lacks the twig-grasping movement, possesses a "bob" component and appears a far "crisper" and more hichly ritualised display than Twigr Shake does.

\subsection{Snap Display}

Common and conspicuous in E. intermedia, Snap is similar to the homolocous displays in Ardea cinerea (Verwey 1930), A. herodies, Butorides virescens, Dichromanassa rufescens and E. thula (Meyerriecks). Head and neck are extended horizontally, then the bird suddenly flexes its legs (the "bob") simultaneously raising its scapular and crest feathers (figure 51). No call or snapping movements of the beak have been observed. The display is often given alternatively with Twig: Shake, although the latter is relatively less frequent. Snap is common in unmated and recently-paired males, and is given by both sexes as part of greetinis ceremonies, particularly during the earlier stages of the pair relationship. Only the bird which has been on the nest performs Snap. Baerends and van der Cingel (1962) have discussed the origin of Snap, which is analysed. furthur: in section 3.76 of this study.

\subsection{Flight Displays}

Circuit flight, Flap Flight Display and Extended Neck 
Flight occur exactly as described for E. garzetta, and the same comments apply. Only one minor difference exists: the "thud" sounds of Flap Flight Display are weaker and more infrequent in E. intermedia

\subsection{Stretch}

All components are identical to those described for E. garzetta, and the display is equally infrequent. No accompanying call has been heard.

\subsection{Greeting Ceremonies}

Only two differences exist between the greeting ceremonies of the two Esretta species. Crest plumes are absent in E. intermedia so crest-feather movements must play a minor part, and the greeting calls have a very different tone in the two species, as already described.

\subsection{Back-bite and Bill-clapper}

Although Back-biting in A. ibis has been distinguished from the Bill-clappering in Ardea cinerea and other species (Hudson 1962), the two displays overlap in E. intermedia. Both displays are common during the first few days of the pair relationship, particularly during greeting ceremonies. The displaying bird runs its beak through its mate's back feathers with quivering mandible movements, sometimes putting its neck across its mate's neck or back. These movements may rapidiy alternate with true Bill-clapper with audible rattling of the mandibles, usually directed above the mate's back, or with the birds necks crossed.

\subsection{Breeding Behaviour.}

The timing of $\mathrm{E}$. intermedia's nesting activities at the Paarl colony in 1966 was very similar to E. garzetta's, except that the former species' last egg was laid between 14 th and 20 th October. All the birds had bright green lores, maby eyes and orange beaks, as described in section 5.1. Unmated birds spent much time flying among the nesting trees, perching at many sites and making many Circuit Flights and Flap Flight Displays, all of which indicates weak site-attachment tendencies, as in E. garzetta. Apart from tinis, the behaviour of unmated $E$. intermedia of both sexes is similar to the behaviour of A. ibis at the same stage. Males adopt and aggressively defend small territories against all 
conspecifics. They spend much time walking up and down their chosen sites with the neck in an "S" position similar to that in Forward Display, and general ruffling of all contour feathers and scapular plumes ocers. Snap, Twig Shake, Stxeteh (rare), Circuit and Flap Flight Displays all occur.

Females attracted to males behaving in this way are characterised by the usual craning and peering movements. Males respond very aggressively towards them, performing Forward Displays and supplanting attacks, occasionally supplanting conspecific females perched up to 10 metres away, while A. ibis are tolerated as close as 2 metres. As in the other two species, each male is repeatedly approached by females which are driven off before one is eventually accepted. The mobile groups of unmated males and females which develop in E. garzetta do not occur in $\mathrm{E}$. intermedia, and the actual moment of pairing has not been observed in either species.

During the pair-formation stage both sexes perch inactively together on the nest for long periods, but seldom in bodily contact as in A. ibis. Snap Display, Back-biting, Bill-clapper and Flap Flight Display all occur, and greeting ceremonies have already been described. As in the other two species, the male collects nest material and the female builds the nest. The copulatory attitude is exactly the same as in E. garzetta and the comments on the subject in that species apply equally to E. intermedia. 


\section{DISCUSSION}

Ethological studies have in recent years been placing less emphasis on detailed motivational analysis of displays. This has possibly come about through a realization that no matter how careful the methods of investigation used, it is impossible ever to confirm conclusions on an animal's motivation. The present author is of the opinion that inotivational analyses of behaviour patterns are of limited use except where coupled with detailed neurophysiological studies, such as in the work on Sticklebacks, rats and some Arthropods, anong others.

A useful product of some descriptive behaviour studies has been their application to systematics. However, behaviour does not lend itself to measurement, so is potentially no more useful than conventional taxonomic characters. One major stumblingblock in comparative studies of display movements is that errors due to subjectivity inevitably arise where different species are studied by different workers. I was particularly alarmed by Meyerriecks' (in Palmer 1962) brief description of the breeding behaviour of A. ibis in Florida. Meyerriecks has considerable experience in Ardeid behaviour yet, according to his descriptions, the behaviour of A. ibis in Florida differs vastly from that observed in the present study: On the basis of behaviour a systematist would probably place the two populations in separate genera! For instance, Meyerriecks states that "No aerial displays were noted", yet Flap Flight Display is the most conspicuous "courtship" display in A. ibis in South Africa. In Stretch the bird "rapidly moves his legs up and down in a dancing motion". In Snap Display (my Twig Shake) the bird "clicks his mandibles together". : In an "upright threat" display "all feathering except crest sleeked (typical)". These descriptions contrast so greatly with those in the present study as to make comparisons meaningless. It is inconceivable that the two populations have diverged so greatly in the century or less that A. ibis has existed in America. The only possible conclusion is that variations in methods of descriptions render this study and Meyerriecks'useless for systematic purposes. These problems do not, of course, exist if all the species under review have been studied in detail by a single competent person, but such studies (e.g. Iorenz 194I, Johnsgard 196I, 1965 and Crook 1964) are comparatively rare. Because so few Ardeidae have been studied to date, and because Meyerriecks is the author 
of the chief work on their behaviour, a review of the family's behaviour would be unwise and is not attempted here.

Another reason why little importance is attached (by the present author) to the value of behaviour studies to systematios is the growing realisation that many behaviour patterns are highly adaptive. Several recent studies have stressed the importance of species ecology in determining the evolution of behaviour. Examples are: Cullen's (1957) investigation of the adaptations of the behaviour of Rissa tridactyla to cliffnesting, von Haartinan's (1957) demonstation that hole-nesting birds show convergence in many characteristics, and Crook's numerous papers (culmination in Crook 1964) on the correlation between reproductive behaviour and ecology in the Ploceinae. In this discussion, no attempt is made to draw comparisons with other Ardeidae, because an attempted synthesis at the present inadequate state of knowledge would be chiefly speculative and thus serve little purpose and compare poorly with the papers mentioned above.

As mentioned in the introduction, A. ibis is a highly successful species, being one of the commonest and most widespread of terrestrial vertebrates. In addion, its colonisation of and vigorous expansion in America (documented by Sprunt 1955 and Davis 1960) has attracted wide attention. One of the stated aims of this study is to investigate advantages of and adaptations to a social way of life in A. ibis and to see whether the species' success is in any way a result of its gregariousness. Before this can be done, the nature of A. ibis' sociability should be reviewed.

In section 5.9 it was consluded that the social life of breeding A. ibis is very simple, and that there is no indication of individual recognition outside of the pair. It was not possible to mark non-breeding birds, but observation on unmarked biras gave no indication of any form of bond between individuals, all interactions being in the form of simple agonistic encounters. For this reason it is proposed to describe birds such as A. ibis only as "gregarious", with the term "social" reservea for birds where bonds and some form of co-operation exist outside of the pair (such as in the wren Malurus cyanura Rowley 1965). A. ibis is highly gregarious at roosts and nesting colonies but only slightly gregarious at its feeding areas. (section 4.I). 
The survival value (if any) of gregariousness while feeding is not discussed here, but presumably some form of local enhancement ("imitation resulting from directing the animal's attention to a particular object or part of the environment", Thorpe 1956) exists, by which the discovery of a temporarily abundant food source by one bird leads to its exploitation by many.

Possible effects of gregarious nesting on reproductive success. in A. ibis will now be investigated. Four points are mentioned, although many minor ones (such as increased probability of parasitism) may exist.

(I) One of the ideas put forward by Darling (1938) was that dense colonial nesting provides social stimulation which in some way improves reproductive success. The idea has been widely discussed but never conclusively proved. No evidence on the subject was sought or found in A. ibis; the point is only mentioned as a possible advantage of gregariousness.

(2) A similar, unconfirmed, theory is that group activities such as roosting play a part in population regulation by enabling animals in some way to obtain information on their density (Wynne-Edwards 1962).

(3) Predation may be affected by gregariousness in a number of ways.

(a) Firstly, nesting colonies are more conspicuous than dispersed nests are, and a concentration of predators could destroy a higher percentage of nests in colonial than in solitary nesting individuals. Predation was low in the present study so it was not possible to draw comparisons in this respect.

(b) Many species have group "mobbing" responses which often distract predators. Mobbing does not occur in A. ibis.

(c) A.ibis' white plumage advertises the fact that predation is unimportant, so one would expect it to have efficient predator-responses. Since no special predator-response (other than loud calls. and in some cases threats) exist in A. ibis, one is forced to conclude that its gregariousness and numbers per se have a deterrent effect on some predators.

(d) Another advantage of gregariousness is that it provides a predator-warning system. A bird in a flock is far less likely to be surprised by a predator than a single bird is. This effect clearly exists in nesting A. ibis, for an outburst 
of alarm calls or of sudden flight results in all birds nearby showing Alert, even if they have not seen the cause of the disturbance.

(4) The chief social implication of colonial nesting is a marked reduction in territory size, as compared with solitary breeders. Considering the evolution of gregariousness: unless aggressive encounters among breeding birds are in some way reduced, an excessive amount of fighting will lower nesting success (cf. Ripley 1961 on "aggressive neglect"), and the evolution of gregariousness would be hindered. There are three ways in which the amount of dysgenic fighting could be reduced.

(a) A highly developed system of threat and submissive displays would reduce fighting. This is almost certainly the case in the Gannets Sula bassana (Nelson 1965) and Morus (Sula) capensis (pers. obs). Both species nest very densely yet have an overriding territorial aggressiveness: threat displays are frequent and fights severe. Comparing $\mathbb{M}$. capensis and A. ibis it is obvious that the former species' displays are relatively far more "distinct", "crisp", of highly ritualised. In addition, appeasement displays are uncommon in A. ibis. I have been repeatedly struck by the low level of agonistic display ritualisation in Ardeidae, as compared to Sulidae and Laridae.

(b) If nest-owners learn to recognise and tolerate neighbours, the amount of fighting will be reduced. Although no figures could be produced on this point in A. ibis, it appeared that nest-owners were equally aggressive towards neighbours and strangers. It is surprising that tolerance of neighbouring nest-owners does not develop in adult A. ibis, for it occurs in the young birds (section 6.32).

(c) If the aggressiveness of a species as a whole is lowered, the amount of fighting would be reduced. From basic principles, it seems unlikely that lower aggressiveness can ever be selected for; one would expect an individual less aggressive than average to stand less chance of obtaining a territory and breeding. Opposing this is the factor of "aggressive neglect" (Ripley 196I), by which very aggressive individuals do not care adequately for eggs and young and thus lower their reproductive turnover. Theoretical considerations apart, the present author obtained the impression that in A. ibis individual distances were much smaller than in the two Egretta species studied. A. ibis is obviously far less aggres- 
sive than Morus capensis, the only colonial non-Ardeid for which the author has first-hand comparison. Allied to this was the observation that $\underline{A}$. ibis are very responsive to conspecific threat displays. This is particularly striking in adult-young agonistic encounters, because Forward Displays of older chicks are invariably successful in driving away trespasising adults. Whatever the evolutionary history of this phenomenon, it iliustrates that the appropriate response to a display can be just as highly ritualised, and just as important in a species' social organisation as the actual display components are themselves. Although the conclusion is unconfirmed and in many respects unsatisfactory, I am convinced that in A. ibis, gregariousness has been promoted by a low level of aggressiveness and/or welldeveloped responsiveness to conspecific threat displays. must be pointed out that if aggressiveness falls below a certain level, reproductive success would almost certainly be lowered)

To conclude, no satisfactory answer could be obtained to the question: Is A. ibis' success in any way a result of its sociality? Part of the species' success must lie in its relative immunity to predation, and although this is to some extent a result of its gregariousness, its immunity remains largely an unsolved problem. Other than a relatively low level of aggressiveness (unproved), no special adaptation of A. ibis' social organisation to colonial nesting was found.

There are 5 outstanding features of A. ibis' biology: its gregariou roosting and nesting habits (widespread in the family); its whiteness (wiaespread in the family); its apparent freedom from predation; its occurrence in terrestrial habitats (only Ardea.melanocephala is more terrestrial); and its comensalistic relationships with large herbivores. Figure 52 illustrates diagramatically all probable relationships between these 5 fieatures.

Considering first the relationship between whiteness and predation; freedom from predation must have favoured the evolution of whiteness (and not vice versa) because as long as predation remains a significant cause of mortality, conspicuousness is selected against. It is generally accepted that conspicuousness is advantageous among conspecifics. On this basis gregariousness favours whiteness. Since whiteness can only evolve when predation is restricted, gregariousness is the factor which enabled the species to become so conspicuous; this is probably the case in many bird species. In seabirds, whiteness is apparently related to feeding methods, making the birds less conspicuous to fish 


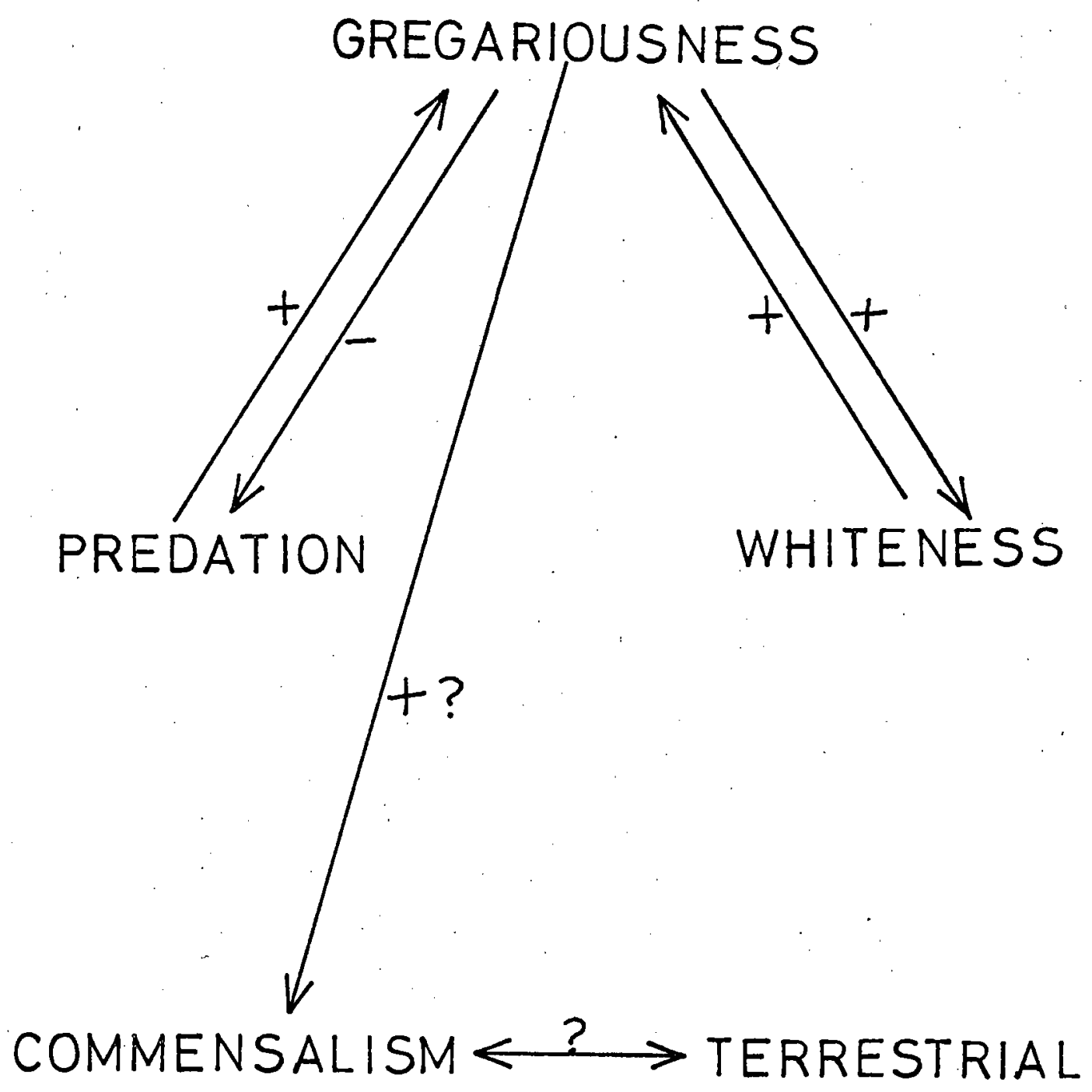

FIGURE 52. Diagram illustrating probable evolutionary relationships between different aspects of A. ibis" biology. + denotes "tends to increase". - denotes "tends to decrease". See text for explanation. 
(Phillips discussed by Tinbergen 1963). It is highly unlikely that A. ibis' colour could affects its success as an insectivore.

The evolution of A. ibis' commensalistic habit may have been favoured by an immunity to predation. Any animal which tendo to associate with an object as conspicuous as a large herbivore automatically makes itself conspicuous as well. Since A. ibis derives predator-protection from its gregarious habits, gregariousness may indirectly favour commensalism.

The potential for commensalism may be widespread in the Ardeidae (Section 2.4). Africa provides optinal conditions for evolution of the habit so it is surprising that no other birds (apart from the Oxpeckers Buphagus and vultures) have become associated with the huge numbers of African herbivores to any great extent. This suggests that A. ibis was in some way specially pre-adapted for commensalism. Any commensal is inevitably affected by its host's biology, and A. ibis' adoption of herbivorous hosts must play some part in the maintenance of its terrestrial habits. There is however no way of deciding whether its commensalistic tendencies originally caused A. ibis to become terrestrial, or vice versa.

This study did not find any unique beñavioural features in A.ibis which could play a role in the vigorous expansion and colonisation which it is at present undergoing. As with other noted vertebrate colonists (eg. Passer domesticus, Rattus norvegicus) A. ibis' range expansion is clearly related to the expansion of man and his activities (Blaker. 1967, Siegfried 1965). In common with these species, it must be, concluded that Ardeola ibis possesses behavioural adaptability to changing conditions. 


\section{A.CKNOWLEDGEIENTS}

My sincere thanks are due to Professor G.J. Broekhuysen, Mrs. M.K. Rowan, Mir.W.R. Siegfried and Professor J.M. Winterbottom for many useful and constructive discussions during the course of this study, and for critically reading the manuscript. C. Farley, W.R. Siegfried, J. Smeda and G.D. Underhill kindly assisted with various aspects of the field work. Lastly, I am very grateful to my mother, Miss L. Briel and Miss R.A. St. I. Searle for patiently bearing the task of typing the manuscript. The work was carried out. while I was at the Percy FitzPatrick Institue of African Ornithology. 


\section{REFERENCES}

Allen, G. M. 1939. A checklist of African mammals. Bull. Mus. Comp. Zool. Harv. 83 1-763

Alley, R. and Boyd, H. 1950. Parent-young recognition in the coot. Ibis 92 46-5I

Almond, W. E. 1955. Display of the Cattle Egret. Brit. Birds 48 453-454.

Andrew, R. J. 1961: Displays given by passerines in courtship and reproductive fighting: a review. Ibis $103 a$ $315-345$

Attwell, R. I. G. 1966. Oxpeckers and their associations with mammals in Zambia. Puku 4 17-48

Baerends, G. P. 1959. The Ethological Analysis of Incubation Behaviour. Ibis 101 357-368

Baerends, G. P. and van der Cingel, N. A. 1962. On the phylogenetic origin of the snap display in: the Common Heron. Symp. Zoo. Soc. Lond. 8 7-24

Bastock, M., Morris, D. and Moynihan, M. 1953. Some comments on conflict and thwarting in animals. Behav. $\underline{6}$ 66-84.

Bent, A. C. 1926. Life histories of North American Marsh Birds. Bull. U.S. Nat. Mus. $1351-490$

Blair, W. F. 1955. Mating call and stage of speciation in the Microhyla olivacea - M. carolinemis complex. Evol. 9. 469

Blaker, D. 1967. Range expansion of the Cattle Egret. Auk. 84, in press.

Bock, W. J. 1956. A generic review of the family Ardeidae (Aves). Am. Mus. Novitates 1779 1-49

Brown, L. H. 1966. Observations on Kenya eagles. Ibis 108 $531-572$

Buerkle, U. and Mansell, W. D. 1963. First nesting record of the Cattle Egret (Bubulcus ibis) in Canada. Auk 80 $378-379$.

Burns, P. S. 1957. Rook and Jackdaw roosts around Bishop's Stortford. Bird Study 4 62-71

Byers, E. 1951. Feeding behaviour of young American Bittern Wils. Bull. 63 334-336.

Cabrera, A. 1960. Catologo de los Mamiferos de America del Sur. Rev. Mus. Argent. Ciene. Nat. 4 (2) 309-739

- Caldwell, D. K. 1956. American Egret feeding with Cattle. Wils. Bull. 6874 
Collias, E. C. and Collias, N. E. 1957. The response of chicks of the Franklin's Gull to parental bill-color. Auk 74 371-375.

Cowles, R. B. 1930. Notes on the nesting of the African Green Heron (Butorides atricapilla) in Natal. Auk 47 $465-470$.

Cott, H. B. 1946. The edibility of birds. Proc. Zool. Soc. Lond. 116 371-542

Cottrille, W. P. and B. D. 1958. Great Blue Heron: Behaviour at the nest. Misc. Publ. Mus. Zool. Univ. Mich. 102 3-15.

Craufard, R. Q. 1966. Notes on the ecology of the Cattle Egret Ardeola ibis at Rokupr, Sierra Leone. Ibis 108 $411-418$.

Crook, J. H. 1963. Comparative studies on the reproductive behaviour of two closely related weaver bird species (Ploceus cucullatus and P. nigerrimus and their races). Behav. 21 177-232.

Crook, J. H. 1964. The evolution of social organisation and visual communication in the weaver birds (Ploceidae) Behav. Suppl. 10. 1-178

Crook, J. H. 1965. The adaptive significance of avian social organisations. Symp. 2001. Soc. Lond. 14 181-218

Cullen, E. 1957. Adaptations of the Kittwake to cliff nesting. Ibis 99 275-302

Culien. J. M. 1962. The pecking response of young Wideawake Terus Sterna fuscata. Ibis 103 b 162-173

Curio, E. 1961. Zur geographischen Variation von Verhaltensweisen. Vogelwelt $82 \quad 33-48$

Curry-Iindahl, K. 1956. Symbios mellen häger (Ardea cinerea) och dovhjort (Dama dama). Vår Fågelvärld 15 $123-126$.

Daanje, A. 1951. On locomotory movements in birds and the intention movements derived from them. Behav. $\underline{3}$ 48-98.

Dane, B., Walcott, C. and Drury, W. H. 1959. The form and duration of the display actions of the Goldeneye (Bucephala bucephala). Behav. 14 265-281.

Darling, F. F. 1938. Bird flocks and the breeding cycle. Cambridge Univ. Press.

Darwin, C. 1872. The expression of the emotions in Man and Animals. London. 
Davis, D.E. 1960. The spread of the Cattle Egret in the United States. Auk. 77, 421-424.

Dobzhansky, T. 1951. Genetics and the origin of species. 3rä è. New Yarke.

Drinkwater, H. 1958. Black-crowned Night Heron using bill motion to Iure prey. Wils. Bull. 70, 201-202.

Ficken, R. W. and Ficken, M. S. 1966. A review of some aspects of avian field ethology. Auk. 83, 637-661.

Haartman, I. von. 1957. Adaptations in hole-nesting birds. Evol. Il, 339-347.

Harford, H. M. 195I. Nest of the Yellow-crowned Night Heron Nyctanassa violacea in Kansas City, Mo. Auk. 68, 235-236.

Harrison; C. J. O. 1965. Allopreening as agonistic behaviour. Behav. 24, 1961-209.

Heatwole, H. 1965. Some aspects of the association of Cattle Egrets with cattie. Anim. Behav. 13, 79-83.

Hediger, H. 1950. Wild Animals in captivity. Iondon. Hinde, R. A. 1952. The behaviour of the Great Tit Parus major and other related species. Behav. Suppl., 2, I-201.

Hinde, R. A. 1955. A comparative study of the courtship of certain finches. Ibis 97, 706-45.

Hinde, R. A. 1959. Behaviour and speciation in birds and lower vertebrates. Biol. Rev. 34, 85-128.

Hudson, M. J. 1965. Bill-clappering display in the Common Heron, Ardea cinerea. Ibis 107, 460-465.

Jenkins, C. F. H. and Ford, J. 1960. The Cattle Egret and its Symbionts in South Western Australia. Emu: 60, 245-9 Johnsgard, P. A. 196I. The taxonomy of the Anatidae - a behavioural analysis. Ibis 103a, 71-85.

Johnsgard, P.A. 1965. Handbook of Waterfowl Behaviour. Cornell Univ. Press, Ithaca, New York. 378 p. Kahl, M. P. 1966. Comparative Ethology of the Ciconiidae. Part 1. The Narabou Stork, Leptoptilos crumeniferus (Lenon). Behav. 27, 76-106.

Kear, J. 1964. Colour preference in young Anatidae. Ibis $106,361-369$.

Kendeigh, S. C. 1952. Parental care and its evolution in birds. Ill. Biol. Monographs 22, 1-356.

lack, D. 1954. The natural regulation of animal numbers. Oxford. 
Lind, H. 1963. Nagle sociale reaktioner hos terner. Dansk. Ornith. For. Tids. 57 155-175.

Lorenz, K. 1941. Vergleichende Bew egungstudein an Anatien. J. Orn. 89 194-294.

Lorenz, K, 1966. On Aggression. London.

Lorenz, K. 1955. Morphology and behaviour patterns in closely allied species. In: "Group Processes" Trans. Ist Conf. 1954 168-220.

Lowe, F..A. 1954. The Heron. Collins, Iondon.

NcKinney, F. 1965. The Comfort movements of Anatidae. Behav. 25 120-220.

Marler, P. 1956. Behaviour of the Chaffinch. Supplement 5 to Behav. 1-184.

Marler, P. 1956. The voice of the Chaffinch and its function as a language. Ibis 98 231-26'l.

Mayfield, H. 1961. Nesting success calculated from exposure. Wils. Bull. 73 255-61.

Mayr, E. 1963. Animal Species and Evolution. Oxford Univ. Press. London.

Meanley, B. 1955. A nesting study of the Little Blue Heron in eastern Arkansas. Wils. Bull. 67 84-99

Meiklejohn, M. F. M. 1952. Ardeidae with red soft parts. Ibis 94544 .

Meyerriecks, A. J. 1960. Comparative breeding behaviour of four species of North American herons. Nuttall Ornith. Club 2 I-158.

Miller, G. S. and Kellogg, R. 1955. List of North American recent mammals. U. S. Nat. Mus. Bull. 205.

Moreau, R. E. and Pitelka, F. A. 1943. Presentation of nesting data. Auk. 60 130-131.

Morris, D. 1954. The reproductive behaviour of the Zebra Finch, with special reference to Mendofemale behaviour and displacement activities. Behav. 6 271-322.

Morris, D. 1956. The feather postures of birds and the problem of the origin of social signals. Behav. 975 .

Moynihan, M. 1955. Remarks on the original sources of displays Auk. $\frac{72}{66} \quad 240-6$

Moynihan, M. 1966. Display patterns of Tropical American "Nine-primarised" songbirds. IV The Yellow-rumped Tanager. Smïths. Misc. Coll. 149 (S) 1-34

Nelson, J. B. 1965. The behaviour of the Gannet. Brit. Birds. 58 (7) 233-287, 313-336. 
Nelson, J. B. 1966a. The breeding biology of the Gannet Sula bassana on the Bass Rock, Scotland. Ibis 108, 584-626.

Nelson, J.B. 1966b. The behaviour of the young Gannet. Brit. Birds 59, 393-418.

Nice, M. M. 1962. Development of Behaviour in Precocial Birds. Irans. Iinn. Soc. N.Y. 1962.

Noble, G.K., Wurm, M. and Schmidt, A. 1938. Social Behaviour of the Black-crowned Night Heron... Auk 55, 7-40.

North, M. E. W. 1963. Breeding of the Black-headed Heron at Nairobi, Kenya, 1958-62. J. E. Afr. Nat. Hist. Soc. $24,33-63$.

Owen, D.F. 1955. The food of the heron Ardea cinerea in the breeding season. Ibis 97, 276-295.

Owen, D. F. 1960. The nesting success of the heron Ardea cinerea in relation to the availability of food. Proc. Zoo. Soc. $133,597-617$.

Palmer, R. S. (ed.) 1962. Handbook of North American Birds. Vol. I, Yale Univ. Press.

Perdeck, A. C. 1958. The isolating value of specific song patterns in two sibling species of grasshoppers (Chorthippus brunneus Thunb. and C. bigutulus L.) Behav. 12, 1-75.

Pettingill, 0. S. 1960. Creche behaviour and individual recognition in a colony of Rockhopper Penguins. Wils. Bull. 72, 213-221.

Rice, D.W. 1954. Symbiotic feeding of Snowy Egrets with cattle in Florida. Auk 71, 472-473.

Rice, D. W. and Kenyon, K. W. 1962. Breeding cycles and behaviour of Laysan and Black-footed Albatrosses. Auk 79, 517-567.

Richale, I. E. 1957. A population study of penouins. Oxford. Ripley, S.D. 196I. Aggressive neglect as a factor in interspecific competition in birds. Auk 78, 366-71.

Rowley, J. 1965. The life history of the Superb Blue Wren Malurus cyanura. Emu 64, 251-297.

Royama, T. 1966. Factors governing feeding rate, food requirement and brood size of nestling Great Tits Parus major. Ibis 108, 313-347. 
Sauer, E. G. F. and E.M. 1966. The behaviour and ecology of the South African Ostrich. Living Bird 5, 45-75.

Siegfried, W.R. 1965. The status of the Cattle Egret in the Cape Province. Ostrich 36, 109-120.

Siegfried, W. R. 1966a. On the food of nestling Cattle Egrets. Ostrich 37, 219-220.

Siegfried, W. R. 1966b. Age at which Cattle Egrets first breed. Ostrich 37, 198-199.

Skead, C. J. 1966. A study of the Cattle Egret Ardeola ibis, Iinnaeus. Proc. 2nd Pan-Afr. Ornith. Cong. 109-139.

Skutch, A. F. 1962. The constancy of incubation. Wils Bull. 74, $115-52$.

Sladen, W. J. I..1953. The Adelie Penguin, Nature 171, 952-955. Sprunt, A. 1955. The spread of the Cattle Egret. Smithsonian Report for 1954. 259-276.

Stonehouse, B. 1960. The King Penguin Aptenodytes patagonica of South Georgia. Falkland Is. Dep. Survey Sci. Rep. 23.

Strijbos, J.P. De Blauwe Reiger. Veen. Amsterdam.

Symrnes, T. C. I. 195I. Display of the Black-necked Heron. Ostrich 22, 38 .

Teal, J.M. 1965. Nesting success of egrets and herons in Georgia. Wils.Bull. 77, 257-263.

Thorpe, W.H. 1956. Learning and Instinct in Animals. Miethuen, Iondon.

Tinbergen, N. 1952. A note on the origin and evolution of threat display. Ibis 94, 160-1.

Tinbergen, N. 1953a. Specialists in nest-building. " Country Life, $30 \mathrm{Jan} ., 270-27 \mathrm{I}$.

Tinbergen, N. 1953b. The Herring Gull's World. Collins, Iondon. Tinbergen, $N$. 1959. Comparative studies of the behaviour of gulls (Laridae): a progress report. Behav. 15, 1-70.

Tinbergen, N. 1960. The evolution of behaviour in gulls. Sci. Amer. (Dec. 1960).

Tinbergen, N. 1963. The work of the animal behaviour research group in the Departiment of Zoology. University of Oxford. Anim. Behav. 11, 206-9.

Tinbergen, N., Broekhuysen, G. J., Feekes, F., Houghton, H., Kruuk, H. and Szulc; E. 1962. Egg shell removal by the Black-headed Gull, Larus ridibundus I.; a 
behaviour component of camoflage. Behav. 19 74-117. Tinbergen, N. and Perdeck, A. C. 1950. On the stimulus situation releasing the begging response in the newly-hatched Herring Gull chick (Larus a. argentatus) Behaviour 3. 1-38.

Tinbergen, N. 1967. Proc. Int. Orn Cong. (in press).

Uys, J. M. C., Clutton-Brock, T. H. 1966. The breeding of the Rufus-bellied Heron (Butorides rufiventris) in Zambia. Puku 4 171-180.

Valentine, J. M. 1958. The Cattle Egret at Chincoteague, Virginia. Raven 29 67-96.

van Iersel. J. J. A. 1953. An analysis of the parental behaviour of the male. Three spined Stickleback (Gasterosteus aculeatus.). Behav. Suppl. 3 1-159.

Verwey, J. 1930. Die Paarungbiologie des Fischreihers. Zool. Jaarb. 48 , $1-120$.

Vince, N. A. 1966. Artificial acceleration of hatching in quail embryos. Anim. Behav. 14 389-394.

Vincent, J. 1947. Habits of the Cattle Egret in Natal. Ibis 89 489-491.

Voous, K. H. 1960. Atlas of European birds. Nelson. Ward, P. 1966. Feeding ecology of the Black-faced Dioch Quelea quelea in Nigeria. Ibis 107 173-214.

Weller, M. W. 1961. Breeding biology of the Least Bittern. Wils. Bull. 73 11-35.

Witherby, H. F., Jourdain, F. C. R., Ticehurst, N. E., and Tucker, B. W. 1940. The Handbook of British Birds. Witherby, London.

Wynne-Edwards, V. C. 1962. Animal dispersion in relation to social behaviour. Oliver and Boyd, Edinburgh. 